(C) 1997 International Press

Adv. Theor. Math. Phys. 1 (1997) 298-387

\title{
Integration Over the $u$-Plane in Donaldson Theory
}

\author{
Gregory Moore ${ }^{a}$, Edward Witten ${ }^{b}$ \\ ${ }^{a}$ Department of Physics, Yale University \\ New Haven, Connecticut 06520, U.S.A. \\ ${ }^{b}$ School of Natural Sciences, Institute for Advanced Study \\ Olden Lane, Princeton, NJ 08540, USA
}

\begin{abstract}
We analyze the $u$-plane contribution to Donaldson invariants of a four-manifold $X$. For $b_{2}^{+}(X)>1$, this contribution vanishes, but for $b_{2}^{+}=1$, the Donaldson invariants must be written as the sum of a $u$ plane integral and an SW contribution. The $u$-plane integrals are quite intricate, but can be analyzed in great detail and even calculated. By analyzing the $u$-plane integrals, the relation of Donaldson theory to $\mathcal{N}=2$ supersymmetric Yang-Mills theory can be described much more fully, the relation of Donaldson invariants to SW theory can be generalized to four-manifolds not of simple type, and interesting formulas can be obtained for the class numbers of imaginary quadratic fields. We also show how the results generalize to extensions of Donaldson theory obtained by including hypermultiplet matter fields.
\end{abstract}

\section{Introduction}

Donaldson theory can be formulated [1] as a twisted version of $\mathcal{N}=2$ supersymmetric Yang-Mills theory. Accordingly, new understanding of $\mathcal{N}=2$ supersymmetric quantum field theory $[2,3]$ has led to new insights about Donaldson theory $[4,5]$. In this paper we continue this development, the main goal being to apply the understanding of supersymmetric Yang-Mills theory to determine the Donaldson invariants of four-manifolds with $b_{2}^{+}=1$. 
Let $X$ be a smooth, compact, oriented four-manifold with Riemannian metric $g$, and let $E \rightarrow X$ be an $S O(3)$ bundle over $X$ (that is, a rank three real vector bundle with a metric). As originally formulated, the Donaldson polynomials are polynomials on the homology of $X$ with rational coefficients:

$$
\mathcal{D}_{E}: H_{0}(X, \mathbb{Q}) \oplus H_{2}(X, \mathbb{Q}) \rightarrow \mathbb{Q} .
$$

Assigning degree 4 to $p \in H_{0}(X, \mathbb{Q})$ and 2 to $S \in H_{2}(X, \mathbb{Q})$, the degree $s$ polynomial may be expanded as:

$$
\mathcal{D}_{E}(p, S)=\sum_{2 n+4 t=s} S^{n} p^{t} d_{n, t}
$$

where $s$ is the dimension of the moduli space $\mathcal{M}$ of instanton connections on $E$.

The numbers $d_{n, t}$ were defined by Donaldson in terms of intersection theory on this moduli space $[6,7,8]$. It is useful to assemble the Donaldson polynomials into a generating function. To do so, one sums over all topological types of bundle $E$ with fixed $\xi=w_{2}(E)$ but varying $p_{1}(E)$ (that is, varying instanton number), to define

$$
\Phi_{\xi}^{X, g}(p, S) \equiv \sum_{n \geq 0, t \geq 0} \frac{S^{n}}{n !} \frac{p^{t}}{t !} d_{n, t} .
$$

This quantity is often the most useful way of organizing the $d_{n, t}$ 's. Here $\Phi_{\xi}$ depends on the characteristic class $w_{2}(E)$ but not on the instanton number $p_{1}(E)$ (as this has been summed over).

If $b_{2}^{+}>1, \Phi$ is independent of the metric $g$ and thus defines "topological invariants" of $X$ (or more precisely invariants of the smooth structure of $X$ ). If $b_{2}^{+}=1, \Phi$ is only piecewise constant as a function of $g[6]$; its detailed dependence on $g$ will be analyzed in section 4. In [1], the Donaldson invariants were identified physically as the correlation functions of certain operators in a topologically twisted $\mathcal{N}=2$ supersymmetric Yang-Mills (SYM) theory with gauge group $S U(2)$ or $S O(3)$. (The $S U(2)$ theory can be regarded as the special case of the $S O(3)$ theory in which one considers an $S O(3)$ bundle $E$ with $w_{2}(E)=0$.) One introduces the fundamental observable

$$
\mathcal{O}(P)=\frac{1}{8 \pi^{2}} \operatorname{Tr} \phi^{2}(P)
$$

where $P$ is a point in $X$, and $\phi$ is a complex scalar field, valued in the adjoint representation of $S U(2)$, and related to the gauge field by supersymmetry. ${ }^{1}$

\footnotetext{
${ }^{1}$ For gauge group $S U(n)$, we mean by $T r$ simply the trace in the $n$-dimensional representation. Equivalently, for $S O(3)$ or $S U(2)$, Tr is $1 / 4$ of the trace in the adjoint representation. With this normalization, $\mathcal{O}$ is related to the restriction to $P \times \mathcal{M}$ of the second Chern class of the universal instanton bundle over $X \times \mathcal{M}$ - in case there is such a universal bundle.
} 
By a fairly standard "descent" procedure, one derives from (1.4) a family of $k$-form valued observables for $k=1, \ldots, 4$. For $X$ simply-connected, the important case is the two-form valued observable

$$
I(S)=\frac{1}{4 \pi^{2}} \int_{S} \operatorname{Tr}\left[\frac{1}{8} \psi \wedge \psi-\frac{1}{\sqrt{2}} \phi F\right] .
$$

We will sometimes refer to $\mathcal{O}$ and $I(S)$ as the zero-observable and twoobservable, respectively.

One of the main results of $[1]$ is that

$$
\Phi_{\xi}^{X, g}(p, S)=\left\langle e^{p \mathcal{O}+I(S)}\right\rangle_{\xi}
$$

where the right hand side is the path integral in a topologically twisted version of the supersymmetric Yang-Mills theory (summed over all $S O(3)$ bundles $E$ with a fixed value of $\xi=w_{2}(E)$ and varying instanton number). This proves to be an effective approach to evaluating Donaldson invariants once one understands the vacuum structure of the supersymmetric gauge theory.

The supersymmetric field theory in question has a family of vacuum states parametrized by a complex parameter $u$ which is defined ${ }^{2}$ by $2 u=\langle\mathcal{O}\rangle$ where here $\langle\mathcal{O}\rangle$ denotes the expectation value computed in a normalized vacuum state on flat $\mathbb{R}^{4}$. As was shown in $[2,3]$, the complex $u$-plane can be identified as the modular curve of the subgroup $\Gamma^{0}(4)$ of $S L(2, \mathbb{Z})$ consisting of integral unimodular matrices whose upper right entry is divisible by four. As such, the complex variable $u$ parametrizes a family of elliptic curves that can be described by a Weierstrass equation ${ }^{3}$

$$
y^{2}=x\left(x^{2}-u x+\frac{1}{4}\right) \text {. }
$$

\footnotetext{
${ }^{2}$ The factor of two in this formula is meant to take care of a slight mismatch in conventions between the mathematical and physical literature on this problem. As written by Kronheimer and Mrowka [9], the "simple type" condition for $X$ reads $\left[\frac{\partial^{2}}{\partial p^{2}}-4\right] \Phi=0$, where $p$ is the variable that appears in the definition of the generating functional $\Phi$. This is an insertion of $\mathcal{O}^{2}-4$ in the correlator. In the physics literature, $u$ is defined so that the discriminant of the elliptic curve that governs the $\mathcal{N}=2$ supersymmetric gauge theory is $u^{2}-1$ (in other words, massless monopoles and dyons appear at the points $u= \pm 1$ where the discriminant vanishes). To reconcile a vanishing discriminant condition $u^{2}-1=0$ with a simple type condition $\mathcal{O}^{2}-4=0$, we require a factor of 2 in the relation between $u$ and $\mathcal{O}$.

${ }^{3}$ This equation describes an elliptic curve with a distinguished subgroup of order four, generated by the points $x=1 / 2, y= \pm \sqrt{(1-u)} / 2$. Note that $\Gamma^{0}(4)$ is conjugate in $G L(2, \mathbb{Q})$ to $\Gamma(2)$, the subgroup of $S L(2, \mathbb{Z})$ consisting of matrices congruent to the identity modulo two. Hence the $u$-plane could be identified (as in [2]) as the modular curve of $\Gamma(2)$, but we use instead (as in [3]) the $\Gamma^{0}(4)$ description (which differs by a two-isogeny), to make some formulas slightly more natural and to facilitate comparison to recent papers such as [10].
} 
The cusps of $\Gamma^{0}(4)$ are the points at $u=\infty, 1$, and -1 where the elliptic curve $C_{u}$ defined by (1.7) degenerates to a rational curve. ${ }^{4}$

To compute Donaldson invariants of a four-manifold $X$ - in other words, to compute certain correlation functions of the twisted $\mathcal{N}=2$ theory on $X$ - one can use any Riemannian metric on $X$. It is convenient to consider the one-parameter family of metrics $g_{t}=t^{2} g_{0}$ with $t \in \mathbb{R}$ and some fixed $g_{0}$. If $t$ is taken large, on general grounds one can compute the correlation functions using a knowledge of the infrared behavior in the various vacua of the theory. If there are only finitely many vacua, one writes the correlation functions as a sum of contributions of the different vacua. In the present case, there is a continuous family of vacua, and one should expect to represent the correlation functions as some sort of "integral" on the $u$-plane.

We have put the word "integral" in quotes because this is not entirely a continuous integral; the measure on the $u$-plane has delta functions supported at $u=1$ and $u=-1$. This occurs because [2] at $u= \pm 1$ there are massless monopoles (or dyons) transforming as hypermultiplets of the supersymmetric theory; the twisted topological theory receives contributions from supersymmetric configurations (obeying the equations $F_{+}=(\bar{M} M)_{+}$, $\Gamma \cdot D M=0$, introduced in [4]) which are possible only at $u= \pm 1$.

Moreover, for many and in some sense most four-manifolds, the contributions from $u= \pm 1$ are the only ones. This, as will become clear, is the physical interpretation of the "simple type" condition [9], which has played an important role in the mathematical analysis of Donaldson theory. In fact, let $b_{i}=b_{i}(X)$ be the Betti numbers of $X$, and write $b_{2}=b_{2}^{+}+b_{2}^{-}$, where $b_{2}^{ \pm}$ are respectively the dimensions of the spaces of self-dual and anti-self-dual harmonic two-forms on $X$. For $b_{2}^{+}>1$, the $u$-plane, away from $u= \pm 1$, does not contribute, as we will show in section 2.3. This is because there are "too many fermion zero modes." Hence for this very large class of four-manifolds, the Donaldson invariants can be written just in terms of monopole solutions, via a formula that is presented in [4] for four-manifolds of simple type, and which we will generalize in section 7 for arbitrary four-manifolds.

Our main interest in the present paper is to explore what happens for $b_{2}^{+}=1$, where the $u$-plane definitely does contribute. (We actually will mainly limit ourselves to the case $b_{1}=0$, although the general case is similar, as we will briefly discuss in section 10 . The $u$-plane will also contribute for $b_{2}^{+}=0$ and $b_{1}$ odd, but this case has a very different flavor and will not be considered here.) The Donaldson invariants are therefore the sum of a continuous integral over the $u$-plane plus delta function contributions from $u= \pm 1$. If we write $Z_{D}$ for a Donaldson theory path integral or correlation function, $Z_{S W}$ for the analogous contribution from monopole

\footnotetext{
${ }^{4}$ Some technical details about elliptic curves and their associated modular functions are collected in Appendix A.
} 
solutions at $u= \pm 1$ (how to obtain the precise formula for $Z_{S W}$ in terms of the conventional monopole or SW invariants will be explained in section 7 ), and $Z_{u}$ for the continuous integral over the $u$-plane, then the general structure is

$$
Z_{D}=Z_{S W}+Z_{u}
$$

We will show that for $b_{2}^{+}=1$, the contribution of the $u$-plane to Donaldson invariants is given by quite complicated-looking integrals which nevertheless, because of their interpretation as integrals over a modular domain, can be analyzed very effectively and even calculated. The integrals involved are similar to integrals that have been studied in work of R. Borcherds in representation theory [11] [12] (and were conjectured in [11] to be related to Donaldson invariants of four-manifolds of $b_{2}^{+}=1$ ) and also in analyses of one-loop threshold corrections in string theory (for example, in [13]).

Once the $u$-plane integrals have been constructed, our analysis of them will involve the following main ingredients:

(i) Homotopy invariance.

(ii) Wall crossing formula.

(iii) Vanishing in certain chambers.

(iv) Behavior under blow-ups.

(v) Explicit evaluation and verification of invariance.

A fuller explanation of these points is as follows.

\section{Homotopy Invariance}

One of the first important points is that the $u$-plane integral $Z_{u}$, despite its considerable complexity and subtlety, depends on only elementary topological information. $Z_{u}$ is completely determined by the cohomology ring of $X$ (in fact, by the intersection form on $H^{2}(X, \mathbb{Z})$ if $X$ is simply-connected). This will be completely clear from the structure of the integrand in the $u$-plane integral.

One therefore gets the same $Z_{u}$ if $X$ is replaced by any four-manifold with the same cohomology ring. For $X$ simply-connected, it follows, given what is known about the intersection pairing on $H^{2}(X, \mathbb{Z})$ for smooth fourmanifolds $X$, that in the evaluation of $Z_{u}, X$ could be replaced by a rational algebra.ic surface, either $\mathbb{P}^{2}$ blown up at $n$ points or $\mathbb{P}^{1} \times \mathbb{P}^{1}$.

\section{Wall Crossing Formula}

For $b_{2}^{+}=1$, the Donaldson "invariants" are not quite invariants $[14,6]$; as the metric of $X$ is varied, $Z_{D}$ generically is constant but "jumps" when certain "walls" are crossed in the space of metrics. Analogous wall-crossing is known for the monopole or SW contributions $Z_{S W}$. (1.8) clearly implies 
that the wall-crossing of $Z_{u}$ is determined by the Donaldson and SW wallcrossing. If we denote the wall-crossing of $Z_{D}, Z_{S W}$, and $Z_{u}$ as $\delta_{D}, \delta_{S W}$, and $\delta_{u}$, then (1.8) implies that

$$
\delta_{D}=\delta_{S W}+\delta_{u} .
$$

This can be better understood as follows. From a physical point of view, it is clear that wall-crossing in $Z_{D}$ must involve the behavior at $u=\infty$. In fact, the proof of invariance under change of metric in the twisted topological field theory involves a fermionic symmetry whose validity depends on integration by parts in field space. Invariance can fail only due to a lack of compactness of field space, which, once one reduces to integration over the space of vacua, means lack of compactness of the $u$-plane. But compactness of the $u$-plane fails only at $u=\infty$. Thus one should aim to understand $\delta_{D}$ in terms of the behavior near $u=\infty$.

On the other hand, as $Z_{S W}$ is supported at $u= \pm 1$, its wall-crossing $\delta_{S W}$ is likewise a contribution from $u= \pm 1$. The structure of $\delta_{u}$ implied by (1.9) is therefore clear; the wall-crossing behavior of the $u$-plane integral must be a sum of a contribution from $u=\infty$ (which in (1.9) will cancel $\delta_{D}$ ) and a contribution from $u= \pm 1$ (which in (1.9) will cancel $\delta_{S W}$ ). This is just the structure we will find.

To be more precise, we will write $\delta_{u}$ as a sum

$$
\delta_{u}=\delta_{u, \infty}+\delta_{u, 1}+\delta_{u,-1},
$$

where the three terms are the contributions to wall-crossing from $u=\infty, 1$, and -1 , respectively. $\delta_{u, \infty}$ will be shown to coincide with the wall-crossing formula for $\delta_{D}$ as determined in greatest generality in $[15,10]$. As for $\delta_{u, 1}$ and $\delta_{u,-1}$, we will see, as expected, that these contributions to wall-crossing are supported exactly where wall-crossing occurs in the monopole or SW invariants.

However, the details of the formulas for $\delta_{u, 1}$ and $\delta_{u,-1}$ involve several universal functions of $u$ (universal in the sense that they do not depend on the choice of four-manifold $X$ ) which have not been computed previously. As we will see in section 7, the same functions arise in expressing Donaldson invariants for hypothetical four-manifolds of $b_{2}^{+}>1$ that are not of simple type in terms of monopole or SW invariants. A knowledge of the formulas for $\delta_{u, \pm 1}$ will determine all the requisite universal functions and enable us to get the general formula for $Z_{S W}$ in terms of monopole or SW invariants, generalizing a formula presented in [4] in the simple type case.

The analysis of $\delta_{u}$ is comparatively easy in that one can calculate the change in $Z_{u}$ upon crossing a wall much more easily than one can actually evaluate $Z_{u}$; the change in $Z_{u}$ in crossing any given wall comes entirely from one relatively simple term in a complicated sum. 


\section{Vanishing in Certain Chambers}

Donaldson invariants of four-manifolds with $b_{2}^{+}=1$ generally do not exhibit simple type behavior. But it is known mathematically that certain $S O(3)$ Donaldson invariants for certain four-manifolds $X$ do exhibit such behavior in certain chambers. This occurs if $X$ maps to a two-dimensional base $B$ with generic fiber $F$ a two-manifold. If $E$ is an $S O(3)$ bundle with $\left(w_{2}(E), F\right) \neq 0$, then simple type behavior is found in a chamber in which the fiber has a very small area relative to the base. If moreover $F$ has genus zero, in which case $X$ is said to be a rational ruled surface, then the Donaldson invariants actually vanish because [17] there are no stable bundles. In this situation, the SW invariants also vanish (because there is a metric of positive scalar curvature), so the $u$-plane integrals must vanish. (Simple type behavior, but not vanishing of the Donaldson or SW invariants, is also found for $F$ of genus one [18].) Simple examples are $X=\mathbb{P}^{1} \times \mathbb{P}^{1}$ or a Hirzebruch surface $\mathbb{F}_{1}$.

Vanishing of the $u$-plane integrals for $X$ a rational ruled surface of very small fiber area and with $\left(w_{2}(E), F\right) \neq 0$ is again an easy consequence of the quantum field theory formulation. Simple type behavior (or at least generalized simple type behavior, the vanishing of $\left(\partial^{2} / \partial p^{2}-4\right)^{r}$ for some integer $r$ ) is a consequence of vanishing of the $u$-plane integral. (This will become clear in section 7.) Vanishing of the $u$-plane integrals for $X$ in the indicated situation follows from pointwise vanishing of the integrand, plus some analysis of behavior near $u=1,-1$, and $\infty$. The pointwise vanishing holds because on the $u$-plane, $S O(3)$ is broken to $U(1)$, and the $S O(3)$ bundle $E$ with $\left(w_{2}(E), F\right) \neq 0$ becomes a line bundle $T$ with $\left(c_{1}(T), F\right) \neq 0$. For any connection on such a line bundle, the "magnetic energy" diverges as the area of $F$ goes to zero, causing the $u$-plane integrand to vanish.

\section{Blow-Up Formula}

By "blowing up" a point in a four-manifold $X$ (or in topological language, taking the connected sum with a copy of $\overline{\mathbb{P}}^{2}$ ), one gets a new four-manifold $\widehat{X}$. The Donaldson invariants of $\widehat{X}$ (in a chamber in which the exceptional divisor $b$ produced by the blow-up has a very small area) are related to those of $X$ by a blow-up formula that has been much studied mathematically $[19,10]$. There are two cases of the blow-up formula, involving $S O(3)$ bundles $E$ with $\left(w_{2}(E), b\right)=0$ and with $\left(w_{2}(E), b\right) \neq 0$.

It is natural to expect that the $u$-plane integral will obey a similar blowup formula. In fact, if there is a universal blow-up formula for $Z_{u}$, it must precisely coincide with the blow-up formula for $Z_{D}$, since it can be determined by considering the special case that $X$ is $\mathbb{P}^{2}$ with a small number of points blown up. Such an $X$ admits a metric of positive scalar curvature, so $Z_{S W}$ vanishes in some chamber; and if $b_{-}(X)<9$, there is no SW 
wall-crossing on $X$ so $Z_{S W}$ actually vanishes everywhere. Hence for such manifolds $Z_{D}=Z_{u}$, so that, if $Z_{u}$ has a blow-up formula of the same general structure as $Z_{D}$, it must be precisely the same formula with the same universal functions.

In fact, we will see that blowing up a point (and assigning a very small area to the exceptional divisor) has a very simple effect on the $u$-plane integrand; by analyzing this effect, we get a blow-up formula for $Z_{u}$ that is in perfect agreement with the blow-up formula for $Z_{D}$ as presented in [10]. This result is easy in the sense that it can be seen before evaluating the $u$-plane integrals; it comes from a relation between the integrands.

\section{Computations}

The basic properties that have been explained up to this point completely determine $Z_{u}$ for all four-manifolds of $b_{2}^{+}=1$ in all chambers, at least for the case that $X$ is simply-connected. Indeed, if $\pi_{1}(X)=0$, one can use the homotopy invariance to reduce to the case that $X$ is a rational surface. Any two rational surfaces, with any two given metrics, can be related to each other by a succession of blow-ups, blow-downs, and wall-crossings. (There is no obstruction to this involving $w_{2}(E)$ since one case of the wall-crossing formula involves a change in $w_{2}$.) So one can reduce to the special case just described of $X=\mathbb{F}_{1}\left(\right.$ or $\left.\mathbb{P}^{1} \times \mathbb{P}^{1}\right)$ in a chamber with $Z_{u}=Z_{S W}=Z_{D}=0$.

The most extensive mathematical computations of Donaldson invariants for four-manifolds of $b_{2}^{+}=1$, such as those in [10], are based on the blow-up and wall-crossing formulas and the vanishing in certain chambers. We will establish all of these properties for the $u$-plane integrals, so we can assert without any detailed calculation that the $u$-plane integrals plus monopole contributions agree with Donaldson theory for rational surfaces.

However, computations based only on blow-up and wall-crossing formulas and reduction to $\mathbb{F}_{1}$ tend to be ineffective in the following sense. To determine any given Donaldson invariant of $X$ by using wall-crossing and blow-up formulas to reduce to a vanishing invariant on $\mathbb{F}_{1}$ involves only finitely many steps. But as one considers Donaldson invariants of $X$ associated with $S O(3)$ or $S U(2)$ bundles of greater and greater instanton number, the number of walls that must be crossed diverges, and it can be hard to get a general and illuminating formula.

One possibility to get effective formulas for $Z_{u}$ is simply to evaluate the integrals. It turns out that, despite their complexity, the integrals defining $Z_{u}$ have special modular properties that make this possible, though the calculations are certainly much harder than the ones alluded to so far.

In this paper we will perform in detail two direct computations of $Z_{u}$. The first is a general computation of $Z_{u}$ for any four-manifold whose intersection 
form contains as a summand the lattice

$$
H=\left(\begin{array}{ll}
0 & 1 \\
1 & 0
\end{array}\right) .
$$

This includes any rational surface except $\mathbb{P}^{2}$ or $\mathbb{F}_{1}$. In this computation we consider bundles with $w_{2}(E)=0$, and certain chambers. This computation is performed using methods of $[11,13]$ together with the Rankin-Selberg method (familiar in string theory $[20,21,22]$ ) of "unwrapping" a modular integral, and certain additional tricks. In this computation, we will explicitly show that, for the chambers we consider, $Z_{u}$ is metric-independent within a chamber. Also, for the case that $X=\mathbb{P}^{1} \times \mathbb{P}^{1}$, we will recover formulas of Göttsche and Zagier [10].

The other computation we perform is for $X=\mathbb{P}^{2}$. This computation depends on techniques of a quite different sort. The main technical tool is a non-holomorphic modular form of weight $3 / 2$, related to Eisenstein series of half-integral weight, that was introduced by Zagier [23, 24]. We will obtain a closed formula for Donaldson invariants of $S U(2)$ bundles on $\mathbb{P}^{2}$, in terms of Hurwitz numbers (essentially class numbers of imaginary quadratic fields). The formula agrees with the special cases that have been computed previously [25] and, when compared with the general expression obtained (via wall crossing and vanishing theorems) by Göttsche [15], yields interesting and perhaps even new formulas for class numbers.

\section{Other u-Plane Integrals}

The basic twisting procedure that relates Donaldson theory to the $S O(3)$ super Yang-Mills theory can be applied to other theories with $d=4, \mathcal{N}=2$ supersymmetry. In the case of the $S U(2)$ theory with "quark" hypermultiplets, the resulting topological field theory involves an integral on the $u$-plane for a family of curves described in [3]. The integral is similar to the case without matter, and can be studied using the techniques discussed above. The results are qualitatively similar for the asymptotically free theories with $N_{f}<4$ flavors. There are some surprises for the asymptotically conformal theories, e.g., for $N_{f}=4$. In this case, there is no wall-crossing for $b_{2}^{+}=1$; a finite set of correlation functions in the theory turn out to be topological, and others vary continuously with the metric.

\section{Organization of the Paper}

This paper is organized as follows. In section two, we present essential physics background. In section three, we work out the detailed form of the $u$-plane integral. In sections four, five, and six we derive the wall-crossing, vanishing, and blow-up properties of this integral. In section seven we use these results to derive the universal form of the SW contributions to the 
Donaldson invariants. In section eight we perform the detailed computation of $Z_{u}$ for four-manifolds whose intersection form contains a summand $H$. In section nine, we compute the $S U(2)$ Donaldson invariants of $\mathbb{P}^{2}$. In section ten we indicate briefly how the results generalize to nonsimply connected manifolds. In section eleven we describe the generalization of these results to topological theories arising from twisting $S U(2) S Y M$ with matter. Some technical details and definitions are collected in appendices A,B,C.

Extension of the $u$-plane integrals considered in the present paper to the case of higher-rank gauge groups (with $I(S)$ still derived from the quadratic Casimir) is under investigation by M. Mariño and G. Moore. Relations between integrable systems and contact terms such as $T(u)$ and its generalizations will be addressed in [26].

\section{Physics Background}

\subsection{Generalities}

We begin with some generalities about $\mathcal{N}=2$ supersymmetric theories in four dimensions. We start out on flat $\mathbb{R}^{4}$, where the double cover Spin(4) of the rotation group is isomorphic to $S U(2)_{-} \times S U(2)_{+}$; the two factors of $S U(2)$ act respectively on the - and + spin representations of $\operatorname{Spin}(4)$, which we call $S_{-}$and $S_{+}$. The $\mathcal{N}=2$ theories of interest here also possess an additional $S U(2)$ group of $R$ symmetries, which we call $S U(2)_{R}$. Under $S U(2)_{-} \times S U(2)_{+} \times S U(2)_{R}$, the supersymmetries transform as $(\mathbf{2}, \mathbf{1}, \mathbf{2}) \oplus$ $(1,2,2)$, where 1 and 2 represent respectively the trivial representation and the two-dimensional representation of $S U(2)$. We introduce $S U(2)_{-}$indices $A, B, C=1,2, S U(2)_{+}$indices $\dot{A}, \dot{B}, \dot{C}=1,2$, and $S U(2)_{R}$ indices $I, J, K=$ 1,2 , and write the supersymmetries as $Q_{A}^{I}$ and $\bar{Q}_{\dot{A} J}$. The coordinates of $\mathbb{R}^{4}$ transform as $(\mathbf{2}, \mathbf{2}, \mathbf{1})$ and will be called $x^{A \dot{A}}$.

The non-zero anticommutators of the $Q$ 's (modulo central terms that will not be important here) are

$$
\left\{Q_{A}^{I}, \bar{Q}_{\dot{A} J}\right\}=4 i \delta^{I} P_{A \dot{A}}
$$

where $P_{A \dot{A}}=\partial / \partial x^{A \dot{A}}$ is the translation generator.

To construct a twisted topological field theory, one introduces a diagonal subgroup $S U(2)^{\prime}$ of $S U(2)_{+} \times S U(2)_{R}$, and one introduces a new action of the Poincaré group of $\mathbb{R}^{4}$ in which rotations act via not $\operatorname{Spin}(4)=S U(2)_{-} \times$ $S U(2)_{+}$but $S p i n(4)^{\prime}=S U(2)_{-} \times S U(2)^{\prime}$. Among the supersymmetries there is the $\operatorname{Spin}(4)^{\prime}$-invariant object $\overline{\mathcal{Q}}=\epsilon^{\dot{A} \dot{B}} \bar{Q}_{\dot{A} \dot{B}}$ and the $\operatorname{Spin}(4)^{\prime}$ vector $K_{A \dot{A}}=-\frac{i}{4} \delta_{A}^{I} Q_{\dot{A} I}$. They obey

$$
\overline{\mathcal{Q}}^{2}=0
$$


and

$$
P_{A \dot{A}}=\left\{\overline{\mathcal{Q}}, K_{A \dot{A}}\right\} .
$$

(2.3) is an integrated version of a formula that asserts locally that the stress tensor $T$ is of the form

$$
T=\{\overline{\mathcal{Q}}, L\},
$$

for some $L$. Note that $K$ obeys

$$
\left\{K_{A \dot{A}}, K_{B \dot{B}}\right\}=0,
$$

on gauge invariant quantities, as a consequence of $(2.1)$.

If the underlying $\mathcal{N}=2$ theory has a $U(2)_{R}$ group of $R$ symmetries (and not just $\left.S U(2)_{R}\right)$, then the center $U(1)_{R}$ is a symmetry of the topologically twisted theory. Under this symmetry, $\mathcal{Q}$ has charge 1 , and $K$ has charge -1 . In Donaldson theory, the $U(1)_{R}$ is a symmetry classically, but quantum mechanically has an anomaly proportional to the dimension of instanton moduli space and is conserved only modulo 8 . The $R$ (or $U(1)_{R}$ ) charge is often called "ghost number" in the context of topological field theory. In the mapping from physical operators to differential forms on instanton moduli space, an operator of ghost number or $R$ charge $q$ is mapped to a $q$-form.

Given a four-dimensional supersymmetric theory with the properties described above, one can aim to formulate the same theory on a general Riemannian four-manifold $X$ in such a fashion that $\overline{\mathcal{Q}}$ is still conserved and (2.2) and (2.4) still hold. This was done for the pure $\mathcal{N}=2$ gauge theory (without hypermultiplets) in [1], and generalized to include hypermultiplets in $[27,28,29]$. The fact that $\overline{\mathcal{Q}}$ is conserved means that one can consistently restrict to $\overline{\mathcal{Q}}$-invariant observables, and the fact that $\overline{\mathcal{Q}}^{2}=0$ means that if one makes this restriction, only the $\overline{\mathcal{Q}}$ cohomology class of a given observable is relevant. The fact that the stress tensor (which is the change in the integrand of the Feynman path integral under a change in metric) is of the form $\{\overline{\mathcal{Q}}, L\}$ means that correlation functions of $\overline{\mathcal{Q}}$-invariant observables are invariant under a change in metric. The theory is therefore a topological field theory.

In constructing the $\overline{\mathcal{Q}}$-invariant observables, an important step is the "descent" procedure, in which one starts with a $\overline{\mathcal{Q}}$-invariant zero-form operator $\mathcal{O}^{(0)}$. By inductively solving the equations

$$
d \mathcal{O}^{(j)}=\left\{\overline{\mathcal{Q}}, \mathcal{O}^{(j+1)}\right\}, \text { for } j=0, \ldots, 3,
$$

one then finds $k$-form valued observables $\mathcal{O}^{(k)}$ for $k=1, \ldots, 4$ which are $\overline{\mathcal{Q}}$ invariant modulo exact forms. This property ensures that for $\Sigma^{(k)}$ a $k$-cycle 
in $X$, the integral

$$
I\left(\Sigma^{(k)}\right)=\int_{\Sigma^{(k)}} \mathcal{O}^{(k)}
$$

is $\overline{\mathcal{Q}}$-invariant and depends only on the homology class of $\Sigma^{(k)}$.

\section{A Canonical Representative}

So far we have merely summarized standard facts about the construction of a certain class of topological field theories. Now we come to a point that is less well-known and is helpful in analyzing the $u$-plane integrals in Donaldson theory. ${ }^{5}$ This is that there is actually a canonical solution of (2.6). That is because of (1.9), which in the twisted topological field theory becomes the statement that there is a one-form valued operator $K$ such that

$$
d=\{\overline{\mathcal{Q}}, K\} .
$$

This means that we can solve (2.6) via

$$
\mathcal{O}^{(j)}=K^{j} \mathcal{O}^{(0)} .
$$

In interpreting the right hand side of (2.9), one understands that $K$ acts on an operator $\mathcal{O}$ by conjugation, that is $K \mathcal{O}$ is shorthand for $[K, \mathcal{O}\}=$ $K \mathcal{O}-(-1)^{\mathcal{O}} \mathcal{O} K$. For $\mathcal{O}$ a zero-form valued operator, the $j$-fold iterated action of $K$ on $\mathcal{O}$ gives an operator, called $K^{j} \mathcal{O}$ in (2.9), that transforms as a $j$-form on $X$; the terms that are not completely antisymmetric (and so do not transform as a $j$-form) vanish according to (2.5).

There are at least two reasons that in the present paper it is useful to have a canonical solution of the descent equations:

(1) The choice of a concrete low energy Lagrangian to describe physics on the $u$-plane is not unique, but is subject to duality transformations that enter the theory in an important way. It is essential to have duality-invariant solutions of the descent equations. The canonical solution, since it can be described without committing oneself to any particular Lagrangian description of the low energy theory, is duality-invariant.

(2) Having this canonical procedure simplifies the task of matching $\overline{\mathcal{Q}}$ invariant operators defined in a microscopic description with $\overline{\mathcal{Q}}$-invariant operators in a macroscopic description. For instance, in the case that the $\mathcal{N}=2$ theory we start with is an $S U(2)$ or $S O(3)$ gauge theory, the basic zero-form observable is $\mathcal{O}=\frac{1}{8 \pi^{2}} \operatorname{Tr} \phi^{2}$, where $\phi$ is a complex field in the adjoint representation that is part of the $\mathcal{N}=2$ vector multiplet. (For gauge theory with a gauge group of rank higher than one, one must also include higher order Casimir invariants of $\phi$.) The expectation value $\langle\mathcal{O}\rangle=2 u$ is the basic order parameter in the low energy theory, ${ }^{6}$ so the operator in the

\footnotetext{
${ }^{5}$ The use of the canonical solution was suggested in this context by N. Seiberg. The existence of a canonical solution to the descent equations was also investigated in [30].

${ }^{6}$ The factor of 2 was explained in a footnote in the introduction.
} 
low energy theory corresponding to the microscopic operator $u$ is "known." Since one also knows what the microscopic supersymmetry generators, and in particular $K$, correspond to in the low energy theory, there is no problem in identifying the descendants $K^{n} u$ as computed in the microscopic theory with corresponding operators in the low energy or macroscopic theory.

\section{Auxiliary Fields}

The last preliminary that we wish to discuss concerns the utility of describing the low energy theory on the $u$-plane in a formalism in which, by including auxiliary fields, the supersymmetry algebra is closed off shell.

In this paper, we will mainly consider simply-connected four-manifolds, so we specialize to the case that the cycles $\Sigma^{(k)}$ of the previous discussion are two-dimensional Riemann surfaces embedded in $X$. We will denote such a Riemann surface as $S$. The existence of a canonical solution of the descent equations enables one to associate with an operator $I(S)=\int_{S} K^{2} u$ of the microscopic theory a corresponding operator $\widetilde{I}(S)=\int_{S} K^{2} u$ in the effective theory on the $u$-plane.

Now we would like to make a similar correspondence for products $I\left(S_{1}\right) I\left(S_{2}\right) \cdots I\left(S_{n}\right)$ with distinct (though perhaps homologous) surfaces $S_{i}$. It is not the case that if a microscopic operator $I\left(S_{i}\right)$ maps to an operator $\widetilde{I}\left(S_{i}\right)$ in the low energy description, then the product $I\left(S_{1}\right) I\left(S_{2}\right) \cdots I\left(S_{n}\right)$ maps to $\widetilde{I}\left(S_{1}\right) \widetilde{I}\left(S_{2}\right) \cdots \widetilde{I}\left(S_{n}\right)$. Rather, at intersections of the $S_{i}$, "contact terms" will appear. ${ }^{7}$ One important simplification is that, as we can pick the $S_{i}$ to have only pairwise intersections, only pairwise contact terms will appear. Moreover, we can assume that the intersections of the $S_{i}$ are generic or "transverse."

The basic structure therefore appears in a product of only two operators:

$$
I\left(S_{1}\right) I\left(S_{2}\right) \rightarrow \widetilde{I}\left(S_{1}\right) \widetilde{I}\left(S_{2}\right)+\sum_{P \in S_{1} \cap S_{2}} \epsilon_{P} T(P) .
$$

Here $T$ is some operator, the sum over $P$ runs over points in the intersection of $S_{1}$ and $S_{2}$, and $\epsilon_{P}$ is \pm 1 depending on whether $S_{1}$ and $S_{2}$ meet with positive or negative orientation at $P$. The operator $T(P)$ must be such that the right hand side of $(2.10)$ is $\overline{\mathcal{Q}}$-invariant and duality-invariant and obeys some more detailed conditions that will be stated later.

If auxiliary fields are included so as to close the supersymmetry algebra off-shell, then the condition for an operator, such as $\widetilde{I}\left(S_{1}\right)$, to be $\overline{\mathcal{Q}}$-invariant is independent of the choice of a specific Lagrangian and in particular is invariant under adding a multiple of $\widetilde{I}\left(S_{2}\right)$ to the action. In that case, if $\widetilde{I}\left(S_{1}\right)$ and $\widetilde{I}\left(S_{2}\right)$ are separately $\overline{\mathcal{Q}}$-invariant, so is their product. If the

\footnotetext{
${ }^{7}$ Such contact terms appeared in [31], for much the same reason, in using $\mathcal{N}=1$ super Yang-Mills theory to compute Donaldson invariants of Kahler surfaces.
} 
supersymmetry algebra is not closed off-shell, then the condition for $\widetilde{I}\left(S_{1}\right)$ to be $\overline{\mathcal{Q}}$-invariant can change if $\widetilde{I}\left(S_{2}\right)$ is added to the action - or, what is closely related, if one takes an operator product with $\widetilde{I}\left(S_{2}\right)$.

Thus, off-shell closure of the supersymmetry algebra makes $\widetilde{I}\left(S_{1}\right) \widetilde{I}\left(S_{2}\right)$ automatically $\overline{\mathcal{Q}}$-invariant, so that the operator $T$ is separately $\overline{\mathcal{Q}}$-invariant. This is a major simplification, and for that reason we will use a formalism in which the supersymmetry algebra is closed off-shell. Of course, in a different formalism, one would obtain equivalent results after lengthier analysis!

In the case of the $u$-plane theory of Donaldson theory, $\overline{\mathcal{Q}}$-invariance of $T$ means (after dropping an irrelevant term of the form $\{\overline{\mathcal{Q}}, \cdot\}$ ) that $T$ is a holomorphic "function" of $u$. We have put the words "function" in quotes for the following reason. We recall from [2] that one of the main points in the understanding of $\mathcal{N}=2$ super Yang-Mills theory is that the low energy theory has many possible Lagrangian descriptions that differ from each other by duality transformations. No one such description is valid globally throughout the $u$-plane. As we will see, the product $\widetilde{I}\left(S_{1}\right) \widetilde{I}\left(S_{2}\right)$, in a formalism with the supersymmetry algebra closed off shell, though $\overline{\mathcal{Q}}$ invariant, is not duality-invariant. As a result, though in any Lagrangian description of the low energy theory $T$ corresponds to a holomorphic function $T(u)$, in order to achieve duality-invariance of the right hand side of (2.10) one must require $T$ to transform non-trivially under duality transformations. After determining the requisite transformation law, we will see that $T$ can be readily and uniquely determined.

Another benefit of holomorphy of $T$ is that it means that in the topological field theory, the point $P$ at which one inserts the operator $T(P)$ (or more precisely $T(u(P)))$ is irrelevant. As a result, once one has determined the object $T$, one can write the formulas in a much more convenient fashion. A useful way to proceed is as follows. Let $S_{i}, i=1, \ldots, b_{2}(X)$ be cycles representing a basis of $H_{2}(X)$; let $\lambda_{i}$ be complex numbers; and let $S$ be a formal sum $S=\sum_{i} \lambda_{i} S_{i}$. Thus $S$ represents an arbitrary element of $H_{2}(X, \mathbf{C})$. We let $S^{2}=\sum_{i, j} \lambda_{i} \lambda_{j} S_{i} \cdot S_{j}$ (where $S_{i} \cdot S_{j}$ is the intersection number of $S_{i}$ and $S_{j}$; thus $S^{2}$ is simply the square of $S$ using the intersection pairing on $H_{2}(X, \mathbf{C})$ ), and we set $I(S)=\sum_{i} \lambda_{i} I\left(S_{i}\right), \widetilde{I}(S)=\sum_{i} \lambda_{i} \widetilde{I}\left(S_{i}\right)$. Then the formula (2.10), together with the absence of higher order contact terms and the separate $\overline{\mathcal{Q}}$-invariance of each term in the formula we are about to write, enables us to put the transformation from microscopic to macroscopic two-observables in its most convenient form:

$$
\exp (I(S)) \rightarrow \exp \left(\widetilde{I}(S)+S^{2} T(u)\right) .
$$

Here the point at which $T$ is inserted is irrelevant, given the $\overline{\mathcal{Q}}$-invariance, so we have written $T(u)$ instead of $T(u(P))$. 


\subsection{The Effective Theory on the $u$-Plane}

We have gotten about as far as we can with generalities. At this point it is time to describe in detail the theory of a single $\mathcal{N}=2$ vector multiplet in four dimensions, of which a special case is the theory of the $u$-plane. (The physical, untwisted model with a general prepotential is described in [32]. The following formulas can be obtained by performing the $\theta$ integrals to reduce to an ordinary Lagrangian and "twisting.")

The bosons in the $\mathcal{N}=2$ vector multiplet are a $U(1)$ gauge field $A$ and a complex scalar $a$ (with its complex conjugate $\bar{a}$ ). The fermions are, in the topologically twisted version of the theory, a zero-form $\eta$, a one-form $\psi$, and a self-dual two-form $\chi$. Under the $U(1)_{R}$ symmetry ("ghost number"), $A$ has charge $0, a$ and $\bar{a}$ have charges 2 and $-2, \eta$ and $\chi$ have charge -1 , and $\psi$ has charge 1 . In order to close the supersymmetry algebra off-shell, one also introduces an auxiliary field $D$; in the topologically twisted theory, $D$ is a self-dual two-form, of $U(1)_{R}$ charge zero. In what follows, given a two-form such as the $U(1)$ field strength $F=d A$, we write $F=F_{+}+F_{-}$, with $F_{+}$and

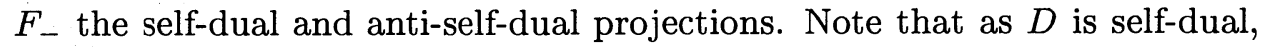
$D=D_{+}$and $D_{-}=0$.

In the topologically twisted model, the $\overline{\mathcal{Q}}$ or BRST transformations are

$$
\begin{aligned}
& {[\overline{\mathcal{Q}}, A]=\psi, \quad[\overline{\mathcal{Q}}, \psi]=4 \sqrt{2} d a,} \\
& {[\overline{\mathcal{Q}}, a]=0, \quad[\overline{\mathcal{Q}}, \bar{a}]=\sqrt{2} i \eta} \\
& {[\overline{\mathcal{Q}}, \eta]=0, \quad[\overline{\mathcal{Q}}, \chi]=i\left(F_{+}-D_{+}\right)} \\
& {[\overline{\mathcal{Q}}, D]=\left(d_{A} \psi\right)_{+},}
\end{aligned}
$$

and the action of $K$ is

$$
\begin{array}{ll}
{[K, a]=\frac{1}{4 \sqrt{2}} \psi,} & {[K, \bar{a}]=0,} \\
{[K, \psi]=-2\left(F_{-}+D\right),} & {[K, A]=-2 i \chi,} \\
{[K, \eta]=-\frac{i \sqrt{2}}{2} d \bar{a},} & {[K, \chi]=-\frac{3 i \sqrt{2}}{4} * d \bar{a},} \\
{[K, D]=-\frac{3 i}{4} * d \eta+\frac{3 i}{2} d \chi .}
\end{array}
$$

The Euclidean Lagrange density is the 4-form:

$$
\begin{aligned}
\frac{i}{6 \pi} K^{4} \mathcal{F}(a)+\frac{1}{16 \pi}\left\{\overline{\mathcal{Q}}, \overline{\mathcal{F}}^{\prime \prime} \chi(D\right. & \left.\left.+F_{+}\right)\right\}-\frac{i \sqrt{2}}{32 \pi}\left\{\overline{\mathcal{Q}}, \overline{\mathcal{F}}^{\prime} d * \psi\right\} \\
& -\frac{\sqrt{2} i}{3 \cdot 2^{5} \pi}\left\{\overline{\mathcal{Q}}, \overline{\mathcal{F}}^{\prime \prime \prime} \chi_{\mu \nu} \chi^{\nu \lambda} \chi_{\lambda}^{\mu}\right\} \sqrt{g} d^{4} x,
\end{aligned}
$$

where $\mathcal{F}(a)$ is a holomorphic function called the prepotential. The free theory (quadratic action) corresponds to the case $\mathcal{F}=\frac{1}{2} \tau_{0} a^{2}$ for some constant $\tau_{0}$. 
Using (2.12) and (2.13) we may expand out (2.14) to get the Lagrange density:

$$
\begin{aligned}
\mathcal{L}= & \frac{i}{16 \pi}\left(\bar{\tau} F_{+} \wedge F_{+}+\tau F_{-} \wedge F_{-}\right)+\frac{1}{2 \pi} \operatorname{Im} \tau d a \wedge * d \bar{a}-\frac{1}{8 \pi}(\operatorname{Im} \tau) D \wedge * D \\
& -\frac{1}{16 \pi} \tau \psi \wedge * d \eta+\frac{1}{16 \pi} \bar{\tau} \eta \wedge d * \psi+\frac{1}{8 \pi} \tau \psi \wedge d \chi-\frac{1}{8 \pi} \bar{\tau} \chi \wedge d \psi+ \\
& +\frac{i \sqrt{2}}{16 \pi} \frac{d \bar{\tau}}{d \bar{a}} \eta \chi \wedge\left(D_{+}+F_{+}\right)-\frac{i \sqrt{2}}{2^{7} \pi} \frac{d \tau}{d a}(\psi \wedge \psi) \wedge\left(F_{-}+D_{+}\right) \\
& +\frac{i}{3 \cdot 2^{11} \pi} \frac{d^{2} \tau}{d a^{2}} \psi \wedge \psi \wedge \psi \wedge \psi-\frac{\sqrt{2} i}{3 \cdot 2^{5} \pi}\left\{\overline{\mathcal{Q}}, \frac{d \bar{\tau}}{d \bar{a}} \chi_{\mu \nu} \chi^{\nu \lambda} \chi_{\lambda}{ }^{\mu}\right\} \sqrt{g} d^{4} x,
\end{aligned}
$$

where $\tau(a)=\mathcal{F}^{\prime \prime}(a)$. In addition there are extra terms

$$
e(u) \operatorname{Tr} R \wedge R^{*}+p(u) \operatorname{Tr} R \wedge R+\frac{i}{4} F \wedge w_{2}(X)
$$

which must be taken into account when coupling to gravity [33]. For the case of the $u$-plane in Donaldson theory, explicit expressions were found for $e(u), p(u)$ in [33]; these expressions are further discussed in section 3.1.

\section{Observables and Contact Term}

We now want to work out the description in the low energy theory of the observable associated with a Riemann surface $S$ and the associated contact term. The mapping of observables from the high energy theory to the low energy theory is

$$
\begin{aligned}
\mathcal{O} \rightarrow 2 u & , \\
I(S) \rightarrow \widetilde{I}(S) & =\frac{i}{\pi \sqrt{2}} \int_{S} K^{2} u \\
& =\frac{i}{\pi \sqrt{2}} \int_{S}\left\{\frac{1}{32} \frac{d^{2} u}{d a^{2}} \psi \wedge \psi-\frac{\sqrt{2}}{4} \frac{d u}{d a}\left(F_{-}+D_{+}\right)\right\} .
\end{aligned}
$$

The two-observable is obtained simply by computing $K^{2} u$, with the above description of $K$ in the twisted theory. The normalization constants in these formulas have been fixed by matching to known results on Donaldson invariants (for instance, the factor of 2 in the first equation is discussed in a footnote in the introduction). In principle, by a more careful understanding of the relation between the $\mathcal{N}=2$ theory as normalized physically and Donaldson theory as defined mathematically, one should be able to make an $a$ priori computation of these normalization factors.

To determine the function called $T(u)$ in (2.11), consider integrating out the auxiliary field $D$ to describe $e^{\widetilde{I}(S)}$ in terms of physical fields only. 
Since the $D$ propagator is $\langle D(x) D(y)\rangle \sim \delta(x, y) / \operatorname{Im} \tau$, this generates a term $\sim(d u / d a)^{2} / \operatorname{Im} \tau$. So after integrating out $D, e^{\widetilde{I}(S)}$ becomes

$$
\exp \left(-\frac{i}{4 \pi} \int_{S}\left(\frac{d u}{d a} F_{-}\right)+\left(S_{+}\right)^{2} \frac{(d u / d a)^{2}}{8 \pi \operatorname{Im} \tau}+\text { fermion terms }\right)
$$

where $S_{+}$is the self-dual part of the cohomology class $S$, and for the moment we need not concern ourselves with the fermion terms.

Equation (2.18) is guaranteed a priori to be $\overline{\mathcal{Q}}$-invariant, but is not modular-invariant. For the terms involving $S_{+}$, the lack of modular invariance is clear in (2.18): it comes because the function $(d u / d a)^{2} / \operatorname{Im} \tau$ is not modular-invariant. However, the full integral involves the contact term discussed previously, and this effectively replaces $e^{\widetilde{I}(S)}$ by

$$
\exp \left(\widetilde{I}(S)+T(u) \cdot S^{2}\right)
$$

where $T(u)$ is a function that will be determined.

The part of the exponent in (2.18) that involves $S_{-}$(the anti-self-dual part of $S)$ is $(d u / d a)\left(S_{-}, F\right)$. The lack of modular invariance here is less obvious. To see it involves analyzing a certain theta function that will enter when we study the $u$-plane integral in detail. We will in due course study this theta function; for now it suffices to note that according to general considerations leading to (2.10) and (2.11), the contact term must, as written in (2.19), be proportional to the intersection number $S^{2}=S_{+}^{2}+S_{-}^{2}$, so that we can determine the contact term by computing the $S_{+}^{2}$ term and then replacing $S_{+}^{2}$ by $S^{2}$.

The contact term will be a holomorphic "function" $T(u)$, appearing in the low energy theory as in (2.19), with the following properties:

(1) In any description by a special coordinate $a$ and photon $A, T(u)$ is a holomorphic function. Under a duality transformation to a different description, $T(u)$ changes in such a way that

$$
\frac{(d u / d a)^{2}}{8 \pi \operatorname{Im} \tau}+T(u)
$$

which is the total coefficient of $S_{+}^{2}$ in the exponent in (2.19), is invariant.

(2) $T$ has no singularity away from cusps in the $u$-plane. The behavior at cusps is as follows. If one works in the appropriate local coordinate near $u= \pm 1$ (for instance, $a_{D}$ at the monopole point, and $a+a_{D}$ at the dyon point) then $T$ has no singularity at $u= \pm 1$. For $u \rightarrow \infty$, if one computes using the special coordinate $a$ which is valid near infinity, then $T / u$ vanishes for $u \rightarrow \infty$.

(3) $T$ is odd under $u \rightarrow-u$. 
The statements in (2) about the behavior of $T$ at cusps are justified as follows. Integrating out massless monopoles or dyons does not produce a singularity in $T$ (we will see in section seven that $T$ coincides with an analogous function $T^{*}$ defined without integrating out the monopoles), so $T$ has no singularity at $u= \pm 1$. The behavior at infinity follows by dimensional analysis and asymptotic freedom. Dimensionally, $T$ takes the form $T=$ $u f\left(\Lambda^{2} / u\right)$, where $\Lambda$ (generally set to 1 in this paper) is the scale parameter of the $\mathcal{N}=2$ theory. $T$ vanishes in the tree approximation, so $f(0)=0$ and hence $T / u$ vanishes for $u \rightarrow \infty$.

Oddness of $T(u)$ under $u \rightarrow-u$ holds because the microscopic $S U(2)$ theory has a classical $U(1)_{R}$ symmetry which is broken to $\mathbf{Z}_{8}$ by a quantum anomaly. Let $w$ be a generator of the $\mathbf{Z}_{8}$ which multiplies a quantum operator of degree (or ghost number, or $R$ charge) $d$ by $e^{2 \pi i d / 8}$. Thus, $w$ maps $u \rightarrow-u$ (because $u=\operatorname{Tr} \phi^{2}$ has $R$ charge four) and $\widetilde{I}(S) \rightarrow i \widetilde{I}(S)$. Thus $T$ must be odd under $w$, that is under $u \rightarrow-u$.

Given these properties, $T$ can be determined as follows. The main point is to determine how the function $G(u)=(d u / d a)^{2} /(8 \pi \operatorname{Im} \tau)$ transforms under $S L(2, \mathbb{Z})$. Under $\tau \rightarrow \tau+1$, that is, $a \rightarrow a$ and $a_{D} \rightarrow a_{D}+a$, clearly $G(u)$ is invariant. Under $\tau \rightarrow-1 / \tau$, we have

$$
\operatorname{Im} \tau \rightarrow \frac{\operatorname{Im} \tau}{\bar{\tau} \tau}
$$

and

$$
\frac{d u}{d a} \rightarrow \frac{d u}{d a_{D}}=\frac{d u / d a}{d a_{D} / d a}=\frac{1}{\tau} \frac{d u}{d a} .
$$

Combining these results, we find that under $\tau \rightarrow-1 / \tau$ we have

$$
G(u) \rightarrow G(u)-\frac{i}{4 \pi \tau}\left(\frac{d u}{d a}\right)^{2} .
$$

Note that the inhomogeneous term in this equation (unlike $G$ itself) is holomorphic in $u$, a crucial property that enables a contact term with the desired properties to exist.

Modular invariance of $G+T$ now amounts to the statement that $T$ is invariant under $\tau \rightarrow \tau+1$ and transforms under $\tau \rightarrow-1 / \tau$ as

$$
T \rightarrow T+\frac{i}{4 \pi \tau}\left(\frac{d u}{d a}\right)^{2} .
$$

A comparison to the standard transformation law for the Eisenstein series $E_{2}(\tau)$ shows that these conditions are equivalent to the statement that

$$
T=-\frac{1}{24} E_{2}(\tau)\left(\frac{d u}{d a}\right)^{2}+H(u)
$$


where $H$ is modular invariant and so is an ordinary holomorphic function of $u$. Conditions (2) and (3) assert that $H$ has no singularities on the finite part of the $u$-plane, grows precisely as $u / 3$ for $u \rightarrow \infty$, and is odd under $u \rightarrow-u$. Hence $H(u)=u / 3$ and

$$
T=-\frac{1}{24}\left(E_{2}(\tau)\left(\frac{d u}{d a}\right)^{2}-8 u\right)
$$

The relation between a microscopic operator $\exp (I(S))$ and macroscopic observables on the $u$-plane is hence

$$
\exp (I(S)) \rightarrow \exp \left(\widetilde{I}(S)+S^{2} T(u)\right)
$$

with $T(u)$ given in (2.26).

\subsection{Vanishing of the $u$-Plane Contribution for $b_{2}^{+}>1$}

We will now establish a fundamental result: the vanishing of the the $u$-plane contribution for four-manifolds with $b_{2}^{+}>1$. In the process we will also learn what contributions do survive for $b_{2}^{+} \leq 1$.

The contribution of the $u$-plane cannot be evaluated by the usual topological field theory technique of reducing to supersymmetric field configurations and then evaluating their contributions. The reduction is usually made by adding to the Lagrangian a term $\lambda\{\overline{\mathcal{Q}}, V\}$, where $\lambda$ is real and $V$ is chosen so that $\{\overline{\mathcal{Q}}, V\}$ vanishes only for supersymmetric configurations, and then taking $\lambda \rightarrow \infty$. In the case of the $u$-plane theory, such a $V$ cannot be chosen in a duality-invariant fashion. In fact, $V$ would have to be chosen to achieve $\tau \rightarrow i \infty$, and this notion is certainly not duality-invariant. (Any choice of $V$ depends on a choice of a particular "photon" multiplet.) Covering the $u$-plane with open sets and using different $V$ 's in different patches would be unhelpful, because the proof of invariance of the correlation functions under addition of $\{\overline{\mathcal{Q}}, V\}$ to the Lagrangian involves an integration by parts in field space which in particular involves integration by parts on the $u$-plane; so one would run into serious complications in the intersections of different patches.

The alternative is to exploit the fact that the theory is expected to be metric-independent (within a chamber, for reasons that will be clear) and to take advantage of this by looking at the behavior in a one-parameter family of metrics $g_{t}=t^{2} g_{0}$, for fixed $g_{0}$, with $t \rightarrow \infty$. What contributions survive as $t \rightarrow \infty$ ? The one-loop determinants of the various fields cancel, by supersymmetry. Almost all Feynman diagram contributions vanish because - the theory on the $u$-plane being unrenormalizable and without marginal or relevant couplings in the renormalization group sense - the vertices scale as negative powers of $t$. We will analyze presently which contributions do 
survive. Finally, in the path integral over abelian connections, one must sum over the various line bundles and the classical solutions (connections with harmonic curvature) on each line bundle. Since free $U(1)$ gauge theory is conformally invariant, the generalized theta function coming from the sum over line bundles survives as $t \rightarrow \infty$; it will be analyzed in some detail later.

To illustrate how the quantum theory works without any technicality, first consider the case that $b_{1}=0$ and $b_{2}^{+}=1$. There is always a single $\eta$ zero mode, with wave-function 1 . For $b_{1}=0$ and $b_{2}^{+}=1$, there are no $\psi$ zero modes and one $\chi$ zero mode. The $\eta$ and $\chi$ zero modes, being zero-forms and two-forms, respectively, are naturally of dimension 0 and 2 . The bosonic fields $F$ and $D$ are of dimension 2. The $\eta$ zero mode and a single $\chi$ zero mode can be soaked up using the terms $\eta \chi D$ or $\eta \chi F_{+}$in the low energy effective action. As these terms are of dimension $0+2+2=4$ and we are in four dimensions, this gives a way to soak up all fermion zero modes with the overall power of $t$ being $t^{0}$. (In doing this, one sets $d \bar{\tau} / d \bar{a}$ to its expectation value at the given point on the $u$-plane.) More explicitly, in performing the path integral, one must sum over line bundles. If $\eta$ and $\chi$ are set equal to harmonic forms, then $\int \eta \wedge \chi \wedge F_{+}$is equal to the integral of the wedge product of the three cohomology classes in question, and is certainly invariant under rescaling of the metric by $g_{0} \rightarrow t^{2} g_{0}$. The $\eta \wedge \chi \wedge D$ term is similar (after integrating out $D$ it will be replaced by $\int_{S} \eta \wedge \chi$, which again depends only on the cohomology classes and not the metric). So these terms give contributions that survive as $t \rightarrow \infty$. As we will see, these are the only contributions that survive.

Suppose that, still with $b_{2}^{+}=1$, we take $b_{1}>0$. We should limit ourselves to the case $b_{1}$ even, since everything vanishes in Donaldson theory unless $1-b_{1}+b_{2}^{+}$is even. There are $b_{1} \psi$ zero modes, and as these are one-forms, they are naturally considered to be of dimension 1 . We can absorb $\psi$ zero modes in groups of four using the interaction vertex $\left(d^{2} \tau / d a^{2}\right) \psi \wedge \psi \wedge \psi \wedge \psi$, and we can absorb $\psi$ zero modes in groups of two using the interaction vertex $(d \tau / d a) \psi \wedge \psi \wedge\left(F_{-}+D\right)$. Either type of vertex gives a factor independent of $t$. Meanwhile the (unique) $\eta$ and $\chi$ zero modes are absorbed by the $\eta \wedge \chi \wedge F$ or $\eta \wedge \chi \wedge D$ terms. So for $b_{2}^{+}=1$ and any even $b_{1}$, there are contributions to the $u$-plane integrand that survive for $t \rightarrow \infty$.

What about $b_{2}^{+}>1$ ? For example, for $b_{1}=0$ and $b_{2}^{+}=3$, one could try to soak up all fermion zero modes using the $\eta \chi^{3}$ term or by using the $\chi^{2}\left(F_{+}-D\right)$ term (along with $\eta \chi\left(F_{+}-D\right)$ ) in the Lagrangian. These additional terms, however, are not topological and in fact scale as $t^{-2}$. Other contributions with the given Betti numbers behave similarly or worse, as we will prove below, so the $u$-plane contribution vanishes for these values of the Betti numbers. The behavior is similar whenever $b_{2}^{+}>1$.

For $b_{2}^{+}=0$ (and $b_{1}$ odd), there are surviving contributions as $t \rightarrow \infty$ 
which actually come from one-loop diagrams. Rather than explaining this in an ad hoc fashion, we will now adopt a more systematic approach.

\section{Scaling}

The non-zero modes in the path integral come in bose-fermi pairs related by $\overline{\mathcal{Q}}$ and so carry a natural measure. Metric independence of the measure thus means that the zero modes (or classical solutions) of the fields $a, A, \eta, \psi, \chi$ should be normalized in a fashion invariant under rescaling of the metric of $X$. (The auxiliary field $D$ has no zero modes.) For example, the expectation value of $a$ determines a point on the $u$-plane; the labeling of such points is completely independent of any metric on $X$. The classical solutions for $A$ (the $U(1)$ connection of the low energy theory) are connections with harmonic curvature on various line bundles over $X$; these are naturally labeled by topological data. The zero modes of $\eta, \psi$, and $\chi$ are harmonic $q$-forms (for $q=0,1$, and 2 respectively) which we take to represent fixed cohomology classes. (We are limited here to speaking of invariance under conformal rescalings of the metric, not under arbitrary changes in metric, since $\chi$ is a self-dual harmonic two-form, whose cohomology class takes values in the self-dual part of $H^{2}(X, \mathbb{R})$, which is invariant under conformal changes of metric on $X$ but not under arbitrary changes.)

Now we expand the various fields as a sum of zero modes plus quantum fluctuations. For instance,

$$
a=a_{0}+a^{\prime}
$$

where $\iota_{0}$ is a constant and $\int_{X} d^{4} x \sqrt{g} a^{\prime}=0$, so that $a^{\prime}$ is orthogonal to the constants or zero modes. Likewise, we set

$$
\begin{aligned}
& \eta=\eta_{0}+\eta^{\prime}, \\
& \psi=\psi_{0}+\psi^{\prime}, \\
& \chi=\chi_{0}+\chi^{\prime}, \\
& A=A_{0}+A^{\prime}
\end{aligned}
$$

where $\eta_{0}, \psi_{0}$, and $\chi_{0}$ are harmonic forms, $A_{0}$ is a connection with harmonic curvature, and $\eta^{\prime}, \psi^{\prime}, \chi^{\prime}$, and $A^{\prime}$ are orthogonal to the space of zero modes.

To analyze the large $t$ behavior, it is convenient to assign dimensions to all fields in such a way that the kinetic energy of all fields has dimension four. There is not a unique way to do this. It is convenient to assign the natural dimensions $1,1,2$ to the bosons $a^{\prime}, A^{\prime}, D$ while assigning dimension 1 to $\psi$ and 2 to $\eta, \chi$. This assignment of dimensions to the fermions differs from the usual choice (dimension $3 / 2$ for all fermions), but still has the property that every term in the fermion kinetic energy is of dimension four.

It is now easy to see that every interaction vertex has dimension at least four, and that every such vertex that contains $\eta$ or $\chi$ fields or contains no 
fermions at all has dimension greater than four. Thus, every dimension four vertex has $\psi$ fields and no other fermions. Since the $\langle\psi \psi\rangle$ propagator vanishes (nonzero fermion propagators are $\langle\eta \psi\rangle$ and $\langle\chi \psi\rangle$ ), all tree and loop diagrams constructed using the quantum fluctuations only vanish for $t \rightarrow \infty$.

What happens if we include insertions of fermion zero modes? (Insertions of bose zero modes just give derivatives with respect to the coupling constants at the various vertices and do not affect the assertions of the last paragraph, which did not depend on details of the couplings.) The zero modes represent fixed cohomology classes and so have geometrical dimensions - dimension $q$ for a $q$-form. Replacing a quantum fluctuation by a fermion zero mode can only help if the zero mode has a smaller dimension than the corresponding quantum fluctuation. The only field for which this is so is $\eta$, which has precisely one zero mode (with constant wave-function). So preciseiy one vertex will be "improved" in its large $t$ behavior by setting $\eta$ equal to a constant. There are three choices for which sort of vertex this might be:

(1) If we set $\eta$ to a constant in the $\eta \chi \chi \chi$ interaction, we find that this interaction still scales as a negative power of $t$. Since all other vertices scale as nonpositive powers of $t$, all contributions of this sort vanish.

(2) We could set $\eta=\eta_{0}$ in the $\eta \wedge \chi \wedge F$ or $\eta \wedge \chi \wedge D$ interaction. These terms then scale as $t^{0}$ regardless of whether for $\chi$ we take a zero mode or a quantum fluctuation. There are now basically three cases:

(a) If $b_{2}^{+}=1$, we must take the $\chi$ field in the $\eta \wedge \chi \wedge F$ or $\eta \wedge \chi \wedge D$ vertex to be a zero mode, since there are no other vertices of dimension four or less that could soak up the $\chi$ zero mode. In the case of the $\eta \wedge \chi \wedge F$ vertex, we must set $F$ equal to a harmonic form; for if $F=d A^{\prime}$ with $A^{\prime}$ a quantum fluctuation while $\eta$ is a constant and $\chi$ is harmonic, then $\int \eta \wedge \chi \wedge F=0$. Otherwise we must use only vertices of dimension four (the alternative being vertices of higher dimension that give vanishing contributions). The only such vertices are $\psi^{4}, \psi^{2} F$, and $\psi^{2} D$; as there is no $\langle\psi \psi\rangle$ propagator, all factors of $\psi$ must be set equal to zero modes (and $F$ to a harmonic form, since if $F=d A^{\prime}$ and the $\psi$ 's are harmonic then $\left.\int \psi \wedge \psi \wedge F=0\right) .{ }^{8}$ This gives the non-vanishing contributions described earlier for four-manifolds with $b_{2}^{+}=1$ and any even $b_{1}$.

(b) If $b_{2}^{+}>1$, there is no way to absorb the $\chi$ zero modes without negative powers of $t$ from vertices of dimension bigger than four.

(c) If $b_{2}^{+}=0$, then in the $\eta \wedge \chi \wedge F$ or $\eta \wedge \chi \wedge D$ vertex, as there are no $\chi$ zero modes, $\chi$ equals a quantum fluctuation $\chi^{\prime}$. (Hence $F$ must likewise be a quantum fluctuation $F=d A^{\prime}$.) There must therefore be additional vertices, and these must be dimension four vertices $\psi^{4}, \psi^{2} F$, or $\psi^{2} D$. As the $\langle\psi \psi\rangle$

\footnotetext{
${ }^{8}$ It can be shown that for $\ddot{b}_{2}^{+}<3$ and all $\psi$ 's equal to zero modes, $\int_{X} \psi^{4}=0$, so this interaction can be dropped.
} 
propagator vanishes, all $\psi$ fields except one must be zero modes. (The $\psi^{4}$ term can hence be dropped for the reason given in the footnote.) By following these rules, one can find several one-loop diagrams that contribute for fourmanifolds with $b_{2}^{+}=0$ and $b_{1}$ odd. These diagrams come from $\eta \chi F \cdot \psi \psi F$ or $\eta \chi D \cdot \psi \psi D$ with a $\langle\chi \eta\rangle$ propagator and either an $\langle F F\rangle$ propagator or a (delta function) $\langle D D\rangle$ propagator.

(3) The remaining possibility is to set $\eta$ to a constant in the $\bar{\tau} \eta d^{*} \psi$ term in the Lagrangian. Since $\int_{X} d^{4} x \sqrt{g} d^{*} \psi=0$, this gives a vanishing contribution if we set $a$ (and hence $\bar{\tau}$ ) to a constant. So the lowest dimension term that arises from this source is a dimension three operator $\overline{a^{\prime}} d^{*} \psi$. Moreover, to get a non-vanishing contribution one must use precisely this vertex, since the dimension four operator $\left(\overline{a^{\prime}}\right)^{2} d^{*} \psi$ would give a negative power of $t$ by the time one absorbs the $\overline{a^{\prime}}$ fields, and higher powers of $\overline{a^{\prime}}$ are of course only worse. So relevant terms of this kind come precisely from the $\overline{a^{\prime}} d^{*} \psi$ operator. In this operator, the $\overline{a^{\prime}}$ field is a quantum fluctuation, of course (rather than a zero mode), and the same is true of $\psi$ as $d^{*} \psi=0$ for $\psi$ a harmonic one-form. So we need to absorb an $\overline{a^{\prime}}$ field and a $\psi^{\prime}$ field using at most one vertex of dimension five (more vertices of dimension five or any of dimension greater than five would give a vanishing contribution for $t \rightarrow \infty$ ) together with dimension four vertices. The only way to do this is to use a dimension five vertex $a^{\prime} d \eta \psi$ (coming from expansion of the $\eta \psi$ kinetic energy in powers of $a^{\prime}$ ) together with any number of dimension four operators $\psi^{4}, \psi^{2} F$, and $\psi^{2} D$. All $\psi$ fields in the dimension five and four vertices just described must be zero modes since there is no $\langle\psi \psi\rangle$ propagator. None of these vertices contain $\chi$ fields, so these terms only contribute for four-manifolds with $b_{2}^{+}=0$ (and any odd $b_{1}$ ). These contributions actually involve the one-loop diagram $\left\langle a^{\prime} d \eta(x) \overline{a^{\prime}} d^{*} \psi(y)\right\rangle$. (The $\psi^{4}$ term can again be dropped.)

In short, certain one-loop diagrams contribute for $b_{2}^{+}=0$, some simple tree diagrams contribute for $b_{2}^{+}=1$, and there are no surviving contributions at all for $b_{2}^{+}>1$. This hierarchy is reminiscent of the progressive simplification found in a certain class of three-dimensional topological field theories as $b_{1}$ is increased [34]. The derivation is also more or less similar.

\section{Explicit Expression for the $u$-Plane Integral}

Our goal in the rest of this paper is to understand the $u$-plane and SW contributions to Donaldson invariants, focussing on the case $b_{1}=0$. (After working out some formal properties of the $u$-plane contributions in sections 4-6, we will then in section 7 analyze the SW contributions to Donaldson invariants.) We would like to calculate the value, in the twisted $\mathcal{N}=2$ theory with gauge group $S O(3)$, of the path integral with an insertion of the 
operator

$$
\exp (p \mathcal{O}+I(S))
$$

where $p$ is a complex number, $\mathcal{O}=\frac{1}{8 \pi^{2}} \operatorname{Tr} \phi^{2}$, and as before $I(S)$ is an arbitrary two-observable. We consider the partition function with this operator inserted summed over $S O(3)$ bundles $E$ with a fixed value of $\xi=w_{2}(E)$. We call this object $\langle\exp (p \mathcal{O}+I(S))\rangle_{\xi}$. It is the generating functional of Donaldson invariants for bundles of the given value of $w_{2}(E)$.

As explained in the introduction, the answer will be the sum of a contribution from the $u$-plane and a contribution from monopole or SW solutions at $u= \pm 1$. In this section, we work out the contribution of the $u$-plane, the analysis of which is the main focus of the present paper. As we have seen, this contribution vanishes for $b_{2}^{+}>1$.

\subsection{Form of the Integral for $b_{2}^{+}=1, b_{1}=0$}

In analyzing the $u$-plane integrals, the first task is simply to write down the $u$-plane integrand for $b_{2}^{+}=1, b_{1}=0$. A number of factors need to be considered, including:

(i) Some interactions that vanish on flat $\mathbb{R}^{4}$ but are present in the twisted theory on a curved four-manifold; and a factor involving the center of the gauge group.

(ii) The integration measure for the zero modes.

(iii) The transformation of (3.1) to the macroscopic theory.

(iv) The path integral of the photons.

(v) The absorption of fermion zero modes and elimination of auxiliary fields.

We consider these factors in turn and then put the pieces together.

\section{Effect of Curved Space}

On the $u$-plane, there are interactions of topological importance that do not appear on flat $\mathbb{R}^{4}$ but do appear if one works on a curved four-manifold $X$. For the case of the topologically twisted theory, these interactions were analyzed in section 3.3 of [33] and multiply the measure by a factor

$$
A^{\chi} B^{\sigma}=\alpha^{\chi} \beta^{\sigma}\left(\left(u^{2}-1\right) \frac{d \tau}{d u}\right)^{\chi / 4}\left(u^{2}-1\right)^{\sigma / 8}
$$

where $\chi$ and $\sigma$ are the Euler characteristic and the signature of $X$, and $\alpha$ and $\beta$ are universal constants (independent of $X$ ) that were not determined in [33]. (These constants could in principle be computed by careful computations in the semiclassical region of large $u$, where asymptotic freedom prevails, but this has not been done.) 
Also, in going from quantum field theory to $S U(2)$ Donaldson theory, there is an extra factor of 2 , because the center of $S U(2)$, which is of order 2 , acts trivially on instanton moduli space; in quantum field theory one divides by this factor of 2 (as part of the Fadde'ev-Popov gauge fixing), but in Donaldson theory it is not customary to do so. ${ }^{9}$

For $b_{1}=0, b_{2}^{+}=1$, we have $\chi+\sigma=4$, so the additional factors in the path integral, including the factor of 2 just mentioned, become

$$
2 \alpha^{\chi} \beta^{\sigma}\left(u^{2}-1\right) \frac{d \tau}{d u}\left(\frac{\left(\frac{d u}{d \tau}\right)^{2}}{u^{2}-1}\right)^{\sigma / 8}
$$

\section{Zero Mode Integration Measure}

With $b_{1}=0$, the bosonic zero modes are purely the choice of a point on the $u$-plane. The metric on the $u$-plane can be read off from the Lagrangian, and is up to a constant multiple $\operatorname{Im} \tau|d a|^{2}$. So the zero mode measure for $a$ is a constant multiple of

\section{$\operatorname{Im} \tau d a d \bar{a}$.}

We need not be precise in determining a universal multiplicative factor here (and similar factors below); this would be part of the determination of the factors $\alpha, \beta$ in (3.3).

There is a single $\eta$ zero mode, with constant wave-function. We write $\eta=$ $\eta_{0}+\eta^{\prime}$, where $\eta_{0}$ is a constant anticommuting $c$-number and $\eta^{\prime}$ is orthogonal to the constants. For $b_{2}^{+}=1$, there is a single $\chi$ zero mode, the wave function being a harmonic self-dual two-form $\omega$ which we normalize so that $\int_{X} \omega \wedge \omega=1$. Note that this condition leaves the sign of $\omega$ undetermined. We write $\chi=\chi_{0} \omega+\chi^{\prime}$, where $\chi_{0}$ is an anticommuting constant and $\chi^{\prime}$ is orthogonal to $\omega$ (we are making a slight change in notation as $\chi_{0} \omega$ was earlier called simply $\chi_{0}$ ). The integration measure for the fermion zero modes is just

$$
\frac{d \eta_{0} d \chi_{0}}{\operatorname{Im} \tau}
$$

The reason for the factor of $\operatorname{Im} \tau$ in the denominator is that the kinetic energy of every field has a factor of $\operatorname{Im} \tau$, which means that the measure for

\footnotetext{
${ }^{9}$ Since we are working on $S O(3)$ bundles of non-zero $w_{2}(E)$, the reader should ask why the center of $S U(2)$ is relevant. The answer is that in standard physical $S O(3)$ gauge theory, one would sum over all values of $w_{2}(E)$; we instead are calculating the value of the path integral for a fixed value of $w_{2}(E)$. This gives a sort of shifted version of $S U(2)$ gauge theory (the standard version of which has $w_{2}(E)$ fixed to be zero). In such a shifted version of the $S U(2)$ theory, the path integral computes the natural topological intersection theory on moduli space, divided by the order of the center of $S U(2)$, just as in the ordinary $S U(2)$ theory.
} 
every bose zero mode has a factor of $(\operatorname{Im} \tau)^{1 / 2}$ and that of every fermion zero mode has a factor of $(\operatorname{Im} \tau)^{-1 / 2}$.

Notice that this measure is odd under a reversal of sign of $\omega$, which changes the sign of $\chi_{0}$. This corresponds to the standard fact $[35,8]$ that defining the sign of the Donaldson invariants requires a choice of a "Donaldson orientation," which is an orientation of $H^{2,+}(X) \otimes H^{1}(X)$. For $b_{1}=0$ and $b_{2}^{+}=1$, a Donaldson orientation is a choice of $\omega$. Our formulas will thus depend on a choice of $\omega$, and will be odd under reversal of sign of $\omega$.

Combining the above, the zero mode measure is simply

$$
d a d \bar{a} d \eta_{0} d \chi_{0}
$$

with no factors of $\operatorname{Im} \tau$.

\section{Observables of the Low Energy Theory}

How to represent the microscopic observable $\exp (p \mathcal{O}+I(S))$ in the low energy theory has already been determined above. The most subtle step was the determination of the contact term $T(u)$ in (2.27). For $b_{1}=0$ and $b_{2}^{+}=1$, the $\psi \psi$ terms in $I(S)$ can be dropped, by arguments similar to those that we gave in explaining the vanishing of the $u$-plane contribution for $b_{2}^{+}>1$. The net result is that

$$
\exp (p \mathcal{O}+I(S)) \rightarrow \exp \left[2 p u-\frac{i}{4 \pi} \int_{S} \frac{d u}{d a}\left(F_{-}+D_{+}\right)+S^{2} T(u)\right],
$$

where the right hand side is to be evaluated in the low energy theory on the $u$-plane.

\section{Photon Path Integral}

An important factor in the $u$-plane integral is the partition function of the free photons. Actually, to be more precise, one will require the photon path integral with an insertion of a certain operator. However, many of the subtleties occur already if one writes simply the photon partition function (which would enter in some physical observables on the $u$-plane, though not in the topological observables considered in the present paper), and we will do this first.

As explained in $[33,36]$, the photon partition function on a four-manifold with $b_{1}=0$ is of the form

$$
Z(\tau)=\frac{\theta_{0}(\tau, \bar{\tau})}{(\operatorname{Im} \tau)^{1 / 2}}
$$

where $\theta_{0}$ is a sort of Siegel-Narain theta function of the lattice $\Gamma=H^{2}(X, \mathbb{Z})$. If one simply took $U(1)$ gauge theory with the action appearing in (2.15) we 
would substitute ${ }^{10} F \rightarrow 4 \pi \lambda$ and this theta function would be

$$
\theta_{0}=\sum_{\lambda \in \Gamma} \exp \left(-i \pi \bar{\tau}\left(\lambda_{+}\right)^{2}-i \pi \tau\left(\lambda_{-}\right)^{2}\right) .
$$

Here $\lambda_{+}$and $\lambda_{-}$are the self-dual and anti-self-dual projections of $\lambda$; hence $\lambda_{+}^{2}>0$ and $\lambda_{-}^{2}<0$. Note that the self-dual projection is explicitly $\lambda_{+}=$ $\omega(\omega, \lambda)$ with $\omega$ the normalized self-dual harmonic two-form introduced above.

To obtain the desired lattice theta function for the $U(1)$ gauge field that appears on the $u$-plane, (3.9) must be modified in two ways. First of all, suppose that we are doing $S U(2)$ gauge theory, spontaneously broken at a generic value of $u$ to $U(1)$. Such breaking means that an underlying $S U(2)$ bundle $W$ reduces to $T \oplus T^{-1}$ where $T$ is a line bundle. The exponent in (3.9) is normalized to be the correct effective action for an $S U(2)$ bundle of this form in Donaldson theory if $\lambda$ is identified as $c_{1}(T)$.

Suppose, however, that one wishes to do $S O(3)$ gauge theory with a bundle $E$ of $w_{2}(E) \neq 0$. In this case, the bundle $W$ does not exist and should be replaced by $E=\operatorname{Sym}^{2}(W)$. However, as far as the low energy theory on the $u$-plane is concerned, the effect of having $w_{2}(E) \neq 0$ is simply that $\lambda$ is no longer an integral cohomology class but is shifted from being an element of $\Gamma$ by $\frac{1}{2} w_{2}(E)$. Thus $\lambda$ is now an element of $\frac{1}{2} \Gamma$ that is congruent to $\frac{1}{2} w_{2}(E)$ modulo $\Gamma$. This shift by itself would turn the theta function into

$$
\theta_{1}=\sum_{\lambda \in \Gamma+\frac{1}{2} w_{2}(E)} \exp \left(-i \pi \bar{\tau}\left(\lambda_{+}\right)^{2}-i \pi \tau\left(\lambda_{-}\right)^{2}\right)
$$

In addition, there is an important phase factor in the lattice sum whose origin was explained in section 4.4 of [33]. This factor may be described as follows. Pick an arbitrary and fixed $\lambda_{0} \in \frac{1}{2} w_{2}(E)+\Gamma$. The factor in question is

$$
(-1)^{\left(\lambda-\lambda_{0}\right) \cdot w_{2}(X)} e^{2 \pi i \lambda_{0}^{2}}
$$

Of course, there is no canonical choice of $\lambda_{0}$ (unless $w_{2}(E)=0$, in which case we take $\lambda_{0}=0$ ). If $\lambda_{0}$ is replaced by $\tilde{\lambda}_{0}$, then (3.11) is multiplied by

$$
(-1)^{\beta \cdot w_{2}(X)}
$$

where $\beta$ is the integral class $\beta=\lambda_{0}-\tilde{\lambda}_{0}$. Thus, with the factor (3.11) included, the overall sign of the Donaldson invariants depends on a choice of $\lambda_{0}$. This fact actually mimics standard facts in Donaldson theory. In Donaldson theory, for $w_{2}(E)=0$, one conventionally orients the instanton

\footnotetext{
${ }^{10}$ Recall that with our conventions, $\lambda \in \frac{1}{2} w_{2}(E)+\Gamma$ is a half-integral class. That is why $F=4 \pi \lambda$ and not $2 \pi \lambda$.
} 
moduli spaces using an orientation of $H^{1}(X) \otimes H^{2,+}(X)$; such an orientation entered our discussion above as a choice of sign for the integration measure of the fermion zero modes on the $u$-plane. When $w_{2}(E) \neq 0$, the conventional way of orienting the instanton moduli spaces depends in addition on a choice of integral lift of $w_{2}(E)$, which in our above notation should be identified with $2 \lambda_{0}$. Moreover, when the integral lift of $w_{2}(E)$ is changed, the usual orientation of the moduli space is multiplied by the factor (3.12). (See [8], pp. 281-3, for a summary of these matters.) Thus, when the factor (3.11) is included, the $u$-plane integral depends on the same choices, and transforms in the same way when the choices are changed, as the orientation of instanton moduli space.

Equally important is the fact that when $\lambda$ is changed by an element of $\Gamma$, the phase factor (3.11) changes by a factor of \pm 1 . Thus, in its dependence on $\lambda$, this phase factor behaves as a sign factor. A field theory explanation of the $\lambda$ dependence of this factor was given in [33], where it was also shown to be crucial in the appearance of $\operatorname{Spin}^{c}$ structures near $u= \pm 1$ after duality. In writing the phase factor precisely as in (3.11), we are fixing an overall, $\lambda$-independent factor that was not analyzed in [33], in such a way as to agree with standard mathematical conventions in Donaldson theory. ${ }^{11}$ Multiplication by a different $\lambda$-independent factor would simply multiply the generating function of the Donaldson invariants by that factor. ${ }^{12}$

Putting these factors together, the photon partition function on the $u$ plane is

$$
Z=\frac{e^{2 \pi i \lambda_{0}^{2}}}{\sqrt{\operatorname{Im} \tau}} \sum_{\lambda \in \Gamma+\frac{1}{2} w_{2}(E)}(-1)^{\left(\lambda-\lambda_{0}\right) \cdot w_{2}(X)} \exp \left(-i \pi \bar{\tau}\left(\lambda_{+}\right)^{2}-i \pi \tau\left(\lambda_{-}\right)^{2}\right)
$$

For application to Donaldson theory, we will actually require not the partition function but the path integral with a certain operator insertion, so it is a related but different function that will appear in the $u$-plane integrals.

\footnotetext{
${ }^{11}$ In particular, that is the reason for the $e^{2 \pi i \lambda_{0}^{2}}$ factor in (3.11). That factor is the same as $e^{i \pi w_{2}(E)^{2} / 2}$, and is completely independent of the choice of $\lambda_{0}$, as $w_{2}(E)^{2}$ has a well-defined value modulo 4 . This factor is included to agree with standard mathematical conventions in Donaldson theory. It would have been equally natural to include instead a factor $e^{-2 \pi i \lambda_{0}^{2}}$. The change would multiply the Donaldson invariants by a sign factor $(-1)^{w_{2}(E)^{2}}$, corresponding to a reversal of the orientation on instanton moduli space. It is necessary to include one or the other factor $e^{ \pm i \pi w_{2}(E)^{2} / 2}$ in order for the Donaldson invariants to come out to be real after performing the $u$-plane integral.

${ }^{12}$ The convention used in [4] actually differed from the present choice (which as we have stated is chosen to agree with standard mathematical conventions) by a sign factor. This sign factor depends on an integral lift of $w_{2}(X)$ and is $(-1)^{\left(2 \lambda_{0}^{2}+\lambda_{0} \cdot w_{2}(X)\right)}$.
} 


\section{Absorption Of Fermion Zero Modes And Elimination Of Auxiliary Field}

For $b_{1}=0$ and $b_{2}^{+}=1$, there are precisely two fermion zero modes. We have already determined in section 2.3 which interactions should be used to absorb these zero modes. The relevant part of the path integral contains precisely one insertion of the $\eta \chi(F+D)$ interaction vertex. This vertex corresponds to a factor in the path integrand that reads

$$
\exp \left[-\frac{i \sqrt{2}}{16 \pi} \int_{X} \frac{d \bar{\tau}}{d \bar{a}} \eta \wedge \chi \wedge\left(F_{+}+D_{+}\right)\right]
$$

Now is a good time to integrate out $D_{+}$and eliminate it from further discussion. The only $D$-dependent factor in the path integral, other than (3.14), appears in (3.7). Combining this with (3.14), the $D$-dependence of the path integral is in a factor

$$
-\exp \left(-\frac{i}{4 \pi} \frac{d u}{d a} \int_{S} D_{+}\right) \cdot\left(\frac{i \sqrt{2}}{16 \pi} \int_{X} \frac{d \bar{\tau}}{d \bar{a}} \eta \wedge \chi \wedge\left(F_{+}+D_{+}\right)\right) .
$$

One can integrate $D$ out of this expression, using the fact that $D$ is a Gaussian field with propagator $\langle D(x) D(y)\rangle \sim \delta(x, y) / \operatorname{Im} \tau$. Upon integrating out $D,(3.15)$ becomes

$$
-\exp \left(S_{+}^{2}\left(\frac{(d u / d a)^{2}}{8 \pi \operatorname{Im} \tau}\right)\right) \cdot\left(\frac{\sqrt{2}}{16 \pi} \int_{X} \frac{d \bar{\tau}}{d \bar{a}} \eta \wedge \chi \wedge\left(F_{+}+i \frac{(d u / d a)}{\operatorname{Im} \tau} S_{+}\right)\right) .
$$

To reduce this further, note that $F_{+}$coincides with $4 \pi \lambda_{+}$. Also, upon integrating over the fermion zero modes, we can replace $\eta$ by 1 and $\chi$ by $\omega$. The resulting factor is hence

$$
-\frac{\sqrt{2}}{4} \frac{d \bar{\tau}}{d \bar{a}} \cdot \exp \left(S_{+}^{2}\left(\frac{(d u / d a)^{2}}{8 \pi y}\right)\right) \cdot\left((\omega, \lambda)+\frac{i}{4 \pi y} \frac{d u}{d a}(\omega, S)\right),
$$

where $\tau=x+i y$.

The first factor in (3.17) depends on the lattice vector $\lambda$ and should be included in defining the lattice sum $\Psi$ that appears in the $u$-plane integrands of Donaldson theory. It is also convenient to include in the definition of $\Psi$ an additional $\lambda$-independent factor of $\exp \left(-S_{-}^{2}(d u / d a)^{2} / 8 \pi y\right)$; this factor is chosen to simplify the modular behavior of $\Psi$. The lattice sum in the $u$-plane integrand is thus

$$
\begin{aligned}
\Psi= & \exp \left[-\frac{1}{8 \pi y}\left(\frac{d u}{d a}\right)^{2} S_{-}^{2}\right] e^{2 \pi i \lambda_{0}^{2}} \sum_{\lambda \in H^{2}+\frac{1}{2} w_{2}(E)}(-1)^{\left(\lambda-\lambda_{0}\right) \cdot w_{2}(X)} \\
& \cdot\left[(\lambda, \omega)+\frac{i}{4 \pi y} \frac{d u}{d a}(S, \omega)\right] \cdot \exp \left[-i \pi \bar{\tau}\left(\lambda_{+}\right)^{2}-i \pi \tau\left(\lambda_{-}\right)^{2}-i \frac{d u}{d a}\left(S, \lambda_{-}\right)\right] .
\end{aligned}
$$


The net effect is that the photon path integral relevant to the $u$-plane integral, combining what appears in (3.8), (3.17), and (3.18), is

$$
Z=-\frac{\sqrt{2}}{4} \frac{d \bar{\tau}}{d \bar{a}} y^{-1 / 2} \exp \left(S^{2} \frac{(d u / d a)^{2}}{8 \pi y}\right) \cdot \Psi .
$$

\section{Putting the Pieces Together}

Combining what we have obtained in (3.6), (3.7), (3.17), and (3.19), the integral over the $u$-plane is simply

$$
Z_{u}=\int_{\mathcal{M}} \frac{d x d y}{y^{1 / 2}} \mu(\tau) e^{2 p u+S^{2} \widehat{T}(u)} \Psi
$$

where $\tau=x+i y, \widehat{T}=T+\frac{1}{8 \pi y}\left(\frac{d u}{d a}\right)^{2}$, and the measure factor is:

$$
\mu(\tau)=-\frac{\sqrt{2}}{2} \frac{d a}{d \tau} A^{\chi} B^{\sigma}=-4 \sqrt{2} i\left(u^{2}-1\right) \frac{d a}{d u}\left(\frac{\left(\frac{2 i}{\pi} \frac{d u}{d \tau}\right)^{2}}{u^{2}-1}\right)^{\sigma / 8} .
$$

We have here fixed the normalization factors $\alpha$ and $\beta$ so as to agree (in the computations that follow) with known results on Donaldson invariants. We have also set $\chi+\sigma=4$, since this is so for four-manifolds of $b_{1}=0, b_{2}^{+}=1$.

Notice that various factors have combined neatly so that the bosonic integration measure $d a d \bar{a}$ in (3.6) is transformed to $d \tau d \bar{\tau}$. This is very convenient since in terms of $a$ there is no reasonable description of the integration region. In terms of $\tau$, however, there is a natural answer: the integration is to be taken over the modular region $\mathcal{M}$ of the group $\Gamma^{0}(4)$, that is, over the quotient $\Gamma^{0}(4) \backslash \mathcal{H}$, where $\mathcal{H}$ is the upper half plane on which the subgroup $\Gamma^{0}(4)$ of $S L(2, \mathbb{Z})$ acts in the usual fashion. This is simply the assertion that the $u$-plane is the modular curve of $\Gamma^{0}(4)$.

The formal proof that this integral (regularized as in the next subsection) is metric-independent follows from the fact that the stress tensor of the twisted theory is of the form $\{\overline{\mathcal{Q}}, \ldots\}$, as a result of which the derivative of the integral with respect to $\omega$ is a total derivative on the $u$-plane. We postpone this argument to section 11.3, where we make this argument in a wider context and show directly that the integral is a locally constant function of $\omega$ with wall-crossing.

\subsection{Definition of the Integral}

At this point we must face the fact that the integral (3.20) does not converge, because of bad behavior near $u=\infty$ and in some cases also near $u= \pm 1$. It is quite clear that if one expands (3.20) in powers of $p$, so as to compute Donaldson invariants of increasing order, then as $u$ diverges at infinity one 
will eventually run into a divergent integral. There is a similar problem near $u= \pm 1$ if $\sigma$ is sufficiently negative.

To complete the definition of the integrals that will be studied in the rest of the paper, we must therefore explain how the divergences will be cut off. We do this in a standard and natural way, as follows. First we expand (3.20) out to a given order in $p$ and $S$, to obtain an integral that should give a Donaldson invariant of some given order. To define that particular integral, after writing $\tau=x+i y$, we perform the integral for $y<y_{0}$, for some cutoff $y_{0}$, and then take the limit as $y_{0} \rightarrow \infty$ only at the end. A similar procedure is followed near the cusps at $u= \pm 1$, introducing the dual $\tau$-parameters at the other cusps and integrating first over $\operatorname{Im} \tau_{D}<y_{0}$, before taking the limit as $y_{0} \rightarrow \infty$. This eliminates the infinities, for the following reason. Set $q=\exp (2 \pi i \tau)$. Then the term in (3.20) that is of any given order in $p$ and $S$ is a sum of terms, each of which is a power of $y$ times a sum of the form

$$
\sum_{\nu, \mu} q^{\nu} \bar{q}^{\mu}
$$

$\nu$ and $\mu$ are not integers (or even rational numbers, in general) but obey $\nu-\mu \in \frac{1}{4} \mathbb{Z}$.

The important point is that, though $\nu$ has no lower bound, $\mu$ is bounded below by zero. The reason for this is that negative exponents in (3.22) come only from factors in $(3.20)$, such as $u$ and $(d \tau / d u)^{-\sigma / 4}$, which are singular at the cusps; these factors are holomorphic and so contribute to $\nu$ but not $\mu$.

Under these conditions, consider an integral of the following form:

$$
\lim _{y_{0} \rightarrow \infty} \int_{y_{1}}^{y_{0}} \frac{d y}{y^{c}} \int_{0}^{k} d x \sum_{\nu, \mu} q^{\nu} \bar{q}^{\mu} .
$$

Here $y_{1}$ is an irrelevant lower cutoff, say $y_{1}=3$, that is included so as to study one cusp while keeping away from others. The interest is in whether the integral converges for $y_{0} \rightarrow \infty$. The $x$ integral runs from 0 to $k$ where (for $\left.\Gamma^{0}(4)\right) k=4$ for the cusp at infinity, and $k=1$ for the other cusps. A detailed examination of (3.20) and of the definition of the function $\Psi$ shows that in all cases either $c>1$ or there are, for a generic metric on $X$ (the metric enters in the definition of $\Psi$ ), no terms with $\nu=\mu=0$. Now integrating first over $x$ projects the sum in (3.23) onto terms with $\nu=\mu$, and hence (as $\mu$ is non-negative) onto terms that vanish exponentially or, if $\nu=\mu:=0$, are constant at infinity. For a generic metric on $X$, the $y$ integral converges as $y_{0} \rightarrow \infty$, since all terms that have survived the $x$ integral have $c>1$ or $\nu, \mu>0$. Via this procedure, the integral becomes for a generic metric a well-defined formal power series in $p, S$. 
For special metrics, on the other hand, there are terms with $c<1$, in fact $c=1 / 2$, and $\nu=\mu=0$. That is where wall-crossing will occur, as we discuss in section 4 .

The cutoff we have given is certainly quite natural and will lead to elegant formulas that agree with Donaldson theory as it has been formulated mathematically. However, one is reluctant to think of any cutoff as fundamental. Since the behavior near $u=\infty$ is linked to the "bubbling" phenomena in Donaldson theory, one might guess that a different but still "reasonable" cutoff might correspond to a different recipe from the one usually used in Donaldson theory for handling the singularities of instanton moduli space. The usual experience in quantum field theory is that upon making a change in the cutoff recipe (within a class of "reasonable" cutoffs) one gets the same theory with a different parametrization. In the present case, for instance, such a reparametrization might mean replacing the function $e^{p u}$ by a function $e^{p u+\alpha(u) \chi+\beta(u) \sigma}$, where $\chi$ and $\sigma$ are the Euler characteristic and signature of $X$ and $\alpha$ and $\beta$ are some universal functions. We will not, however, investigate the extent to which either the cutoff-dependence of the $u$-plane integrals, or the dependence of Donaldson theory on how the singularities are treated, can be so written.

Curiously, the regularization relevant to Donaldson theory also coincides with that needed to define one-loop amplitudes in string theory. In particular (3.20) bears a striking resemblance to threshold corrections in compactifications of heterotic string on $\mathrm{K} 3 \times S^{1}$. It would be interesting to understand this more deeply.

\subsection{Verification of Modular Invariance}

Having defined the integration at the cusps it is still worth checking that the integral actually makes sense, namely, that the integrand is single-valued. This is equivalent to checking modular invariance of the integrand under $\Gamma^{0}(4)$. Verifying this is a test of our calculation since the underlying $S U(2)$ gauge theory is intrinsically defined, but to compute the explicit $u$-plane integrand we have had to use an effective low energy $U(1)$ description that is only uniquely determined up to a modular transformation.

Modular invariance is most readily checked by relating $\Psi$ in (3.20) to the standard Siegel-Narain theta functions which transform simply under modular transformations. Our notation is explained in Appendix B. We introduce the theta function

$$
\Theta=\kappa^{-\left(w_{2}(X), w_{2}(E)\right)} \Theta_{H^{2}}\left(\tau, \frac{1}{2} w_{2}(X), \frac{1}{2} w_{2}(E) ; P_{\omega}, \xi\right),
$$


with $\kappa=e^{2 \pi i / 8}$ and

$$
\xi=\rho y \frac{d \bar{a}}{d \bar{u}} \omega+\frac{1}{2 \pi} \frac{d \bar{u}}{d \bar{a}} S_{-} .
$$

Defining

$$
\widehat{f}(p, S, \tau, y) \equiv \mathcal{N}\left(\left(u^{2}-1\right) \frac{d \tau}{d u}\right)^{\chi / 4}\left(u^{2}-1\right)^{\sigma / 8} \frac{d u}{d \tau} \exp \left[2 p u+S^{2} \widehat{T}(u)\right]
$$

for an appropriate normalization constant $\mathcal{N}$, we now introduce the auxiliary integral $\mathcal{G}(\rho)$

$$
\mathcal{G}(\rho) \equiv \int_{\Gamma^{0}(4) \backslash \mathcal{H}} \frac{d x d y}{y^{3 / 2}} \widehat{f}(p, S, \tau, y) \bar{\Theta},
$$

related to the Coulomb partition function by:

$$
Z_{u}=\left.(S, \omega) \mathcal{G}(\rho)\right|_{\rho=0}+\left.2 \frac{d \mathcal{G}}{d \rho}\right|_{\rho=0} .
$$

Denote the integrand of (3.27) by $\frac{d x d y}{y^{2}} \mathcal{J}$, where $\mathcal{J}=\widehat{f} \cdot y^{1 / 2} \bar{\Theta}$. We obtain a fundamental domain for $\Gamma^{0}(4)$ from a fundamental domain $\mathcal{F}$ for $P S L(2, \mathbb{Z})$ by

$$
\Gamma^{0}(4) \backslash \mathcal{H} \cong\left[\mathcal{F} \cup T \cdot \mathcal{F} \cup T^{2} \cdot \mathcal{F} \cup T^{3} \cdot \mathcal{F}\right] \cup S \cdot \mathcal{F} \cup T^{2} S \cdot \mathcal{F}
$$

The first four domains give the region of the cusp at $\tau \rightarrow i \infty$ and correspond to the semiclassical region. The region $S \cdot \mathcal{F}$ surrounds the cusp near $\tau=0$ and will be referred to as the monopole cusp. The region $T^{2} S \cdot \mathcal{F}$ surrounds the cusp near $\tau=2$ and corresponds to the massless dyon.

Mapping the integrand in these 6 regions to the domain $\mathcal{F}$ we get six functions:

$$
\begin{aligned}
\mathcal{J}_{(\infty, 0)}(\tau) & \equiv \mathcal{J}(\tau) \\
\mathcal{J}_{(\infty, 1)}(\tau) & \equiv \mathcal{J}(\tau+1) \\
\mathcal{J}_{(\infty, 2)}(\tau) & \equiv \mathcal{J}(\tau+2) \\
\mathcal{J}_{(\infty, 3)}(\tau) & \equiv \mathcal{J}(\tau+3) \\
\mathcal{J}_{M}(\tau) & \equiv \mathcal{J}(-1 / \tau) \\
\mathcal{J}_{D}(\tau) & \equiv \mathcal{J}(2-1 / \tau)
\end{aligned}
$$

In general we will denote $\Gamma^{0}(4)$-modular forms $F$ transformed as in (3.30) by $F_{I}$ where

$$
I=(\infty, 0),(\infty, 1),(\infty, 2),(\infty, 3), M, D
$$


These will form representations of the permutation group $S_{3} \cong \bar{\Gamma} / \Gamma^{0}(4) \cdot{ }^{13}$

It is now straightforward to bring the integral to the form

$$
\mathcal{G}(\rho)=\int_{\mathcal{F}} \frac{d x d y}{y^{3 / 2}} \sum_{I} \widehat{f}_{I}(p, S, \tau) \bar{\Theta}_{I}
$$

where

$$
\Theta_{I}=e^{i \phi_{I}} \Theta_{H^{2}}\left(\tau, \alpha_{I}, \beta_{I} ; \xi_{I}\right),
$$

are the transforms of the Siegel-Narain theta function implied by (3.30). It is easy to check that $\widehat{f}_{I}$ and $\Theta_{I}$ transform in the same unitary representation of the modular group. Hence $\mathcal{G}$ is modular invariant, and therefore, so is $Z_{u}$.

\subsection{The Four Basic Properties}

Here and in sections 4-6 we will examine, in light of what we have learned, the basic formal properties mentioned in the introduction.

The homotopy invariance of the $u$-plane integral is manifest from the form of the integrand in (3.20). The $u$-plane integral for a simply-connected four-manifold is completely determined by the lattice $\Gamma=H^{2}(X, \mathbb{Z})$ with its intersection pairing. Thus, these integrals, while extremely subtle, capture only elementary topological information. Only because there are additional contributions from $u= \pm 1$ is this compatible with the fact that Donaldson invariants of four-manifolds contain further information beyond the intersection form. Those contributions involve the SW invariants and will be the subject of section 7 .

As explained in the introduction, beyond homotopy invariance, the $u$ plane integrals possess three additional formal properties that determine them completely. These are the chamber dependence, the vanishing in certain chambers, and the blow-up formulas. The next three sections are devoted to these properties in turn.

\section{Chamber-Dependence of $Z_{u}$ and Wall-Crossing Formulae}

We are now in a position to give a comparatively simple explanation of the wall-crossing formula for the $u$-plane integrals. It is useful to think in terms of the analogy of (3.20) to one-loop integrals in string theory. In this analogy the wall-crossing discontinuities arise when - in the language of

\footnotetext{
${ }^{13}$ Actually, one encounters modular forms of half-integer weight and hence occasionally one must work with the metaplectic double-cover.
} 
string amplitudes - a massive particle becomes massless on a subvariety of Narain moduli space, leading to an infrared divergence in the integral.

In the present integral, there are three cusps $\tau=i \infty, \tau=0, \tau=2$. The first cusp leads to the Donaldson wall-crossing formulae. The other two lead to the SW wall-crossing formulae.

As explained in section 3.2, any discontinuity in $Z_{u}$ arises from a finite number: of terms in $\Psi$ and from negative powers of $q$ in the Fourier expansion of the nearly holomorphic modular forms. The relevant terms are of the form

$$
\mathcal{I}(\omega) \equiv \int_{\mathcal{F}} \frac{d x d y}{y^{1 / 2}} c(d) e^{2 \pi i x d-2 \pi y d} e^{-i \pi x\left(\lambda_{+}^{2}+\lambda_{-}^{2}\right)} e^{-\pi y\left(\lambda_{+}^{2}-\lambda_{-}^{2}\right)}(\omega, \lambda),
$$

for some integer $d$ and some $\lambda$. (The $u$-plane integrand also contains additional terms proportional to $y^{-3 / 2}$ instead of $y^{-1 / 2}$ and lacking the factor $(\omega, \lambda)$. It will, however, become clear that such terms produce no singularity.) In (4.1), $c(d)$ is the coefficient of some modular object. It is also a function of $p$ and $\left(S_{-}, \lambda_{-}\right),\left(S_{+}, \lambda_{+}\right)$, but this has not been indicated explicitly.

We want to study the integral in (4.1) for fixed $\lambda$ as the decomposition $\lambda=\lambda_{+}+\lambda_{-}$varies. We recall that $\lambda_{+}=\omega(\omega, \lambda)$, so this decomposition is determined by $\omega$. The issue is to find a discontinuity in $\mathcal{I}(\omega)$ when a "wall" is crossed. Such a discontinuity occurs only if $\lambda^{2}<0$, since otherwise the integral in (4.1) (with the regularization described in section 3.2) is too well-behaved to produce a discontinuity. When, however, $\lambda^{2}<0$, there is a discontinuity at $\lambda_{+}=0$. This can be computed as follows. Upon doing the $x$ integral, one projects onto $d$ such that $2 d=\lambda^{2}$. For this value of $d$, the $y$ integral looks like

$$
\int_{y_{1}}^{\infty} \frac{d y}{y^{1 / 2}} c\left(\lambda^{2} / 2\right) e^{-2 \pi y \lambda_{+}^{2}} \lambda_{+}
$$

This is an elementary integral (if one replaces $y_{1}$ by 0 ) and converges for all non-zero $\lambda_{+}$, but is discontinuous at $\lambda_{+}=0$. The discontinuity comes from the large $y$ part of the integral and so is independent of $y_{1}$. The discontinuity in $\mathcal{I}(\omega)$ as $\omega$ crosses from $(\omega, \lambda)=0^{-}$to $(\omega, \lambda)=0^{+}$is easily computed to $\mathrm{be}^{14}$

$$
\mathcal{I}\left(\omega_{+}\right)-\mathcal{I}\left(\omega_{-}\right)=\sqrt{2} c(d)=\sqrt{2}\left[q^{-\lambda^{2} / 2} c(q)\right]_{q^{0}} .
$$

The notation $[\cdot]_{q^{0}}$ indicates the constant term in a Laurent expansion in powers of $q$. It may also be expressed as a residue. Since $\lambda_{+}=0$ we may put $\left(S_{+}, \lambda_{+}\right)=0$ and $\left(S_{-}, \lambda_{-}\right)=(S, \lambda)$ in the function $c(q)$.

\footnotetext{
${ }^{14}$ This is the contribution of a single copy of the $S L(2, \mathbb{Z})$ fundamental domain near the cusp. For the cusp at infinity, there is an extra factor of four from the summation over four copies of the fundamental domain, or equivalently from the fact that $x$ runs from 0 to 4 .
} 
The conditions $\lambda^{2}<0, \lambda_{+}=0$ for a discontinuity are very familiar in Donaldson theory. They are the conditions that the line bundle with Chern class $\lambda$ admits an instanton connection, which (upon embedding of $U(1)$ in $S U(2)$ or $S O(3))$ appears as a singular point in instanton moduli space. The discontinuity of $Z_{D}$ is usually computed by studying the behavior near this singularity. The conditions $\lambda^{2}<0, \lambda_{+}=0$ for a wall are also very natural in string theory. They are the conditions that in toroidal compactification of the heterotic string, a massive particle with Narain vector $\lambda$ becomes massless and gives an infrared singularity. The above computation exhibiting the discontinuity has a direct analog in heterotic string threshold computations. See, for examples, $[13,37]$.

The equation (4.3) is of central importance. It shows (modulo an analysis we give presently showing that the contributions of the different cusps do not cancel) that the partition function $Z_{u}$ is not topologically invariant. Indeed, the conditions $\lambda^{2}<0, \lambda_{+}=0$ define chambers in the forward light cone $V_{+}=\left\{\omega \in H^{2}(X ; \mathbb{R}): \omega^{2}>0\right\}$. Any $\lambda$ with $\lambda^{2}<0$ defines a wall in $V_{+}$by

$$
W_{\lambda} \equiv\{\omega: \lambda \cdot \omega=0\} .
$$

The chambers are the complements of the walls.

When $\omega$ crosses such a wall there is a discontinuity in $Z_{u}$ given by (4.3) for an appropriate $c(q)$. Any given correlation function $\sim\left\langle\mathcal{O}^{\ell} \mathcal{I}(S)^{r}\right\rangle$ will involve an integral with a holomorphic form with pole growing linearly with $r, \ell$. Thus, any such correlation function will only be piecewise constant as a function of $\omega$. The number of chambers for such a correlator grows with $r, \ell$. Let us now examine in more detail the chamber-dependence coming from the singularities at the three cusps of $\Gamma^{0}(4) \backslash \mathcal{H}$.

\section{Comparison to Donaldson Theory Wall-Crossing Formulas}

The four cosets forming the cusp at $\tau \rightarrow i \infty$ contribute the semiclassical wall-crossing formula. As explained in the introduction, this contribution should coincide with the wall-crossing formula for the Donaldson invariants.

For these cosets, the shifts $\beta_{I}$ defined in (3.33) are all given by $\beta_{I}=$ $\frac{1}{2} w_{2}(E)$ and the formula (4.3) for the quantity $\delta_{u, \infty}$ of equation (1.10) becomes:

$$
\begin{aligned}
& Z_{u,+}-Z_{u,-}=-32 i(-1)^{\left(\lambda-\lambda_{0}, w_{2}(X)\right)} e^{2 \pi i \lambda_{0}^{2}} \\
& \cdot\left[q^{-\lambda^{2} / 2}\left(u^{2}-1\right) h(\tau)\left(\frac{\left(\frac{2 i}{\pi} \frac{d u}{d \tau}\right)^{2}}{u^{2}-1}\right)^{\sigma / 8} \exp \left\{2 p u+S^{2} T(u)-i(\lambda, S) / h\right\}\right]_{q^{0}} .
\end{aligned}
$$

Here $h \equiv \frac{d a}{d u}$. In appendix A we give expressions for the various modular forms in (4.5) in terms of Jacobi $\vartheta$-functions. Using these expressions (4.5) 
simplifies to: ${ }^{15}$

$$
\begin{aligned}
Z_{u,+}-Z_{u,-}= & -\frac{i}{2}(-1)^{\left(\lambda-\lambda_{0}, w_{2}(X)\right)} e^{2 \pi i \lambda_{0}^{2}} \\
& \cdot\left[q^{-\lambda^{2} / 2} \frac{\vartheta_{4}^{8+\sigma}}{h(\tau)^{3}} \exp \left\{2 p u+S^{2} T(u)-i(\lambda, S) / h\right\}\right]_{q^{0}},
\end{aligned}
$$

with $h=\frac{1}{2} \vartheta_{2} \vartheta_{3}$ and

$$
u=\frac{1}{2} \frac{\vartheta_{2}^{4}+\vartheta_{3}^{4}}{\left(\vartheta_{2} \vartheta_{3}\right)^{2}} .
$$

In fact, the semiclassical wall-crossing formula (4.6) is identical to the wall-crossing formula given in $[15,10]$ for Donaldson invariants of fourmanifolds with $b_{1}=0, b_{2}^{+}=1$. In making this comparison we must note the following. Modular forms not of $\Gamma^{0}(4)$ but of a group conjugate to it by $\tau \rightarrow \tau+1$ are used in $[15,10]$, and one must make this transformation in (4.6) before matching the modular forms. (The shift $\tau \rightarrow \tau+1$ does not affect the residue or $q^{0}$ coefficient in (4.6).)

\section{SW Wall Crossing}

We will now analyze the wall-crossing behavior associated with the monopole and dyon cusps at $\tau=0,2$. As explained in the introduction, this wallcrossing contribution is related to the wall-crossing behavior of SW invariants - a connection that we will explore further in section 7 .

The "monopoles" that appear near $u=1$ are not spinors (sections of a spin bundle $S_{+}$) but are sections of a $\operatorname{Spin}^{c}$ bundle which we write somewhat symbolically as $S_{+} \otimes L$, where $L$ does not exist as a line bundle but $L^{\otimes 2}$ does. We define $\lambda=\frac{1}{2} c_{1}\left(L^{2}\right)$; thus, $\lambda \in \frac{1}{2} w_{2}(X)+\Gamma$. Hence, in the previous notation, $\beta=-\frac{1}{2} w_{2}(X)$ for these cusps. The walls are still defined by

$$
\begin{aligned}
\lambda^{2} & <0, \\
(\lambda, \omega) & =0 .
\end{aligned}
$$

At the monopole cusp, the formula (4.3) for the discontinuity $\delta_{u, 1}$ of equation (1.10) becomes:

$$
\begin{aligned}
Z_{u,+}-Z_{u,-} & =-\frac{i}{8} e^{2 i \pi\left(\lambda_{0} \cdot \lambda+\lambda_{0}^{2}\right)} \\
\cdot & {\left[q^{-\lambda^{2} / 2} \frac{\vartheta_{2}^{8+\sigma}}{\left(h_{M}\right)^{3}} \exp \left\{2 p u_{M}+S^{2} T_{M}-i(\lambda, S) / h_{M}\right\}\right]_{q^{0}} }
\end{aligned}
$$

\footnotetext{
${ }^{15}$ The right hand side of (4.5) is odd under $\lambda \rightarrow-\lambda$. Therefore at any wall the contributions of $\pm \lambda$ to the discontinuity in $Z_{u}$ add, rather than cancel because this is a formula for $Z_{+}-Z_{-}$.
} 
where

$$
\begin{aligned}
h_{M} & =\frac{1}{2 i} \vartheta_{3} \vartheta_{4}, \\
u_{M} & =\frac{1}{2} \frac{\vartheta_{3}^{4}+\vartheta_{4}^{4}}{\left(\vartheta_{3} \vartheta_{4}\right)^{2}}, \\
T_{M} & =-\frac{1}{24}\left[\frac{E_{2}}{h_{M}(\tau)^{2}}-8 u_{M}\right] .
\end{aligned}
$$

There is a similar expression for the dyon cusp. Recall that $\lambda_{0}$ is a fixed element of $\frac{1}{2} w_{2}(E)+\Gamma$ (or in other words that $2 \lambda_{0}$ is a fixed integral lift of $w_{2}(E)$ ) which entered in defining the $u$-plane integrals in section 3 (and which in the usual mathematical theory enters in orienting the instanton moduli spaces).

We would like to stress that the functions $u, h, T$ in (4.8) are the same functions occuring in the integrand of $Z_{u}$; however, they are most usefully expressed in terms of the expansion relevant to the cusp at $\tau=0$, namely the expansion in powers of $q_{D} \equiv \exp \left(2 \pi i \tau_{D}\right)$ where $\tau_{D} \equiv-1 / \tau$. To avoid cluttering the notation, we have simply written $\tau, q$ in (4.8).

It is interesting to derive a condition for (4.8) to be nonvanishing. Defining

$$
d_{\lambda}=\frac{1}{4}\left[(2 \lambda)^{2}-\left(9-b_{-}\right)\right]
$$

we see that the leading power of $q$ in (4.8) is $q^{-\frac{1}{2} d_{\lambda}}$. Thus,

$$
d_{\lambda} \geq 0
$$

is a necessary condition for SW wall-crossing. In particular, note that we must have $8+\sigma=9-b_{-}<0$. As we explain in section 7 below, (4.11) is in perfect correspondence with SW theory. Indeed, $d_{\lambda}$ is the virtual dimension of SW moduli space. We will show that a knowledge of the wall crossing formula for the cusps at $u= \pm 1$ even allows us to learn about Donaldson invariants for (hypothetical) four-manifolds of any $b_{2}^{+}$that are not of simple type.

\section{Vanishing in Certain Chambers}

Another important property of the $u$-plane integrals is that they vanish for certain four-manifolds $X$ in certain chambers, for $S O(3)$ bundles $E$ with certain values of $w_{2}(E)$. In conjunction with the wall-crossing formulae explored in section 4 , this determines the values of $Z_{u}$ for such $X, E$. 
We recall from the introduction that the appropriate four-folds $X$ are "rationally ruled surfaces," which map to a two-dimensional base $B$, with generic fiber $F$ of genus zero. The required bundles are bundles such that $\left(w_{2}(E), F\right) \neq 0$. The vanishing chamber is defined by the requirement that the area of the fibers goes to zero. As we will see, the vanishing occurs because the lattice theta function vanishes in this limit, and hence the $u$ plane integrand vanishes pointwise.

To fix ideas, we will focus on the Hirzebruch surface $\mathbb{F}_{1}$. The general case is similar. Let us set up some notation. We regard $X=\mathbb{F}_{1}$ as a blow-up of projective space $\mathbb{F}_{1}=B l_{P}\left(\mathbb{P}^{2}\right)$. The blow-up produces an exceptional divisor $B$. $X$ fibers over $\mathbb{P}^{1}$ with $\mathbb{P}^{1}$ fibers which we call $F$, and $B$ is a section of this fibration. There are two natural bases for $H^{2}(X ; \mathbb{Z})$. One basis consists of the pair $\langle F, B\rangle$. In this basis, the intersection form is:

$$
\left(\begin{array}{cc}
0 & 1 \\
1 & -1
\end{array}\right)
$$

Alternatively, we can introduce $H=B+F$, the pullback of a hyperplane class on $\mathbb{P}^{2}$. In the basis $\langle H, B\rangle$, the intersection form is

$$
\left(\begin{array}{cc}
1 & 0 \\
0 & -1
\end{array}\right)
$$

We choose an integral lift of $w_{2}(X)$ by setting $w_{2}(X)=F$. The Kahler cone is $\{x B+y H: x \leq 0, x+y \geq 0\}$. Any Kahler metric of unit volume has a Kahler class of the form

$$
\omega=\cosh \theta H-\sinh \theta B, \quad 0 \leq \theta<\infty,
$$

$\omega$ is a self-dual harmonic two-form with $(\omega, \omega)=1$. Define $\epsilon \equiv e^{-\theta}$. We are interested in the limit

$$
\begin{aligned}
& \omega \cdot F=\epsilon \rightarrow 0, \\
& \omega \cdot B=\sinh \theta \rightarrow \infty,
\end{aligned}
$$

in which the area of the fibers becomes small. This is the limit in which vanishing will occur.

The basic reason for the vanishing is the following. Suppose that instead of $\mathbb{F}_{1}$ we took $X=\mathbb{P}^{1} \times \mathbb{P}^{1}=B \times F$. (This choice of $X$ - being fibered over $B=\mathbb{P}^{1}$ by the projection of $B \times F$ to the first factor - is in any case a perfectly acceptable example of the class of manifolds for which the vanishing result holds.) To analyze the lattice theta function in this case, note that if $B \times F$ is given a product metric, then a harmonic two-form on $B \times F$ is 
the sum of a pullback from $B$ and a pullback from $F$. The Maxwell action is easily seen to be:

$$
\int_{B \times F} F \wedge * F=\frac{\operatorname{vol}(B)}{\operatorname{vol}(F)}\left(\int_{F} F\right)^{2}+\frac{\operatorname{vol}(F)}{\operatorname{vol}(B)}\left(\int_{B} F\right)^{2} .
$$

Therefore, if the flux $\int_{F} F$ is forced to be nonvanishing, which will be the case if $\left(w_{2}(E), F\right) \neq 0$, and $\frac{\operatorname{vol}(B)}{\operatorname{vol}(F)} \rightarrow \infty$, then the action goes to infinity and the path-integral is suppressed. Confining a nonzero magnetic flux in a small fiber costs a lot of action.

For $\mathbb{F}_{1}$ or a more general rationally or elliptically fibered manifold, the metric is not such a simple product. Nevertheless, the same basic idea holds. For instance, in the case of $\mathbb{F}_{1}$, if we take $w_{2}(E)=B$ (a special case of $\left.\left(w_{2}(E), F\right) \neq 0\right)$, then upon reduction to the abelian theory on the $u$-plane one gets line bundles of first Chern class

$$
\lambda=n H+\left(m+\frac{1}{2}\right) B, \quad \text { with } n, m \in \mathbb{Z} .
$$

The gauge theory action for such a line bundle is:

$$
\begin{aligned}
& \exp \left[-i \pi \bar{\tau} \lambda_{+}^{2}-i \pi \tau \lambda_{-}^{2}\right]= \\
& \quad \exp \left[-\pi y\left[n^{2}+\left(m+\frac{1}{2}\right)^{2}\right] \cosh 2 \theta-i \pi x\left[n^{2}-\left(m+\frac{1}{2}\right)^{2}\right]\right] .
\end{aligned}
$$

Since $n, m$ are integral, (5.7) always leads to an exponential suppression in the limit (5.4). The only other metric dependence in the integrand comes from the terms:

$$
\exp \left[\frac{1}{8 \pi y} S_{+}^{2} / h^{2}\right] \exp \left[-i\left(S_{-}, \lambda\right) / h\right][(S, \omega)+4 \pi i y(\lambda, \omega) / h] .
$$

Now,

$$
\begin{aligned}
(S, \omega) & =\cosh \theta S \cdot F+e^{-\theta} S \cdot B . \\
\left(S, \omega^{\perp}\right) & =-\sinh \theta S \cdot F+e^{-\theta} S \cdot B .
\end{aligned}
$$

To any given order in $S$, this extra metric dependence contributes at most a power $1 / \epsilon^{N}$. This is killed by the exponential suppression of the terms (5.7) in the $\Psi$-function. Therefore, the contribution of the integration over any compact region vanishes for $\epsilon \rightarrow 0$.

To show vanishing of the $u$-plane integral in the given limit, pointwise vanishing of the integrand is not quite enough. This is because the integration region is noncompact and the convergence is not uniform throughout 
the $u$-plane. Therefore, to complete the argument, we must make some more careful estimates at the three cusps.

The cusp at $\infty$ is easily handled. To study the behavior near the cusp, we can replace the integral over $\mathcal{F}$ by the integral over the strip $-\frac{1}{2} \leq x \leq$ $+\frac{1}{2}, y \geq 1$. We focus on a term giving a fixed power $p^{\ell} S^{r}$. The expression (5.7) multiplies a modular form times a polynomial in $1 / y$. After the projection $\int d x$ the integral has an absolute value bounded above by:

$$
\begin{aligned}
\sum_{n, m \in \mathbb{Z}}|c(d(n, m))| & \sum_{N, M} \frac{a_{N, M}}{\epsilon^{M}} \int_{1}^{\infty} \frac{d y}{y^{1 / 2}} y^{-N} \\
\cdot & \exp \left[-2 \pi y n^{2}(\cosh \theta)^{2}-2 \pi y\left(m+\frac{1}{2}\right)^{2}(\sinh \theta)^{2}\right],
\end{aligned}
$$

where the modular form is $\sum c(d) q^{d}, 2 d(n, m)=n^{2}-\left(m+\frac{1}{2}\right)^{2}, N, M$ are nonnegative integers, and the number of terms in the sum $\sum_{N, M}$ is bounded by $r$ for the contribution to $p^{\ell} S^{r}$. Now we simply use the estimate:

$$
\int_{1}^{\infty} \frac{d y}{y^{1 / 2}} y^{-N} \epsilon^{-M} \exp \left[-y \frac{K}{\epsilon^{2}}\right] \sim \frac{1}{\epsilon^{M-2}} \exp \left[-\frac{K}{\epsilon^{2}}\right]\left(1+\mathcal{O}\left(\epsilon^{2}\right)\right) .
$$

The contribution of the monopole and dyon cusps requires a little more care. The modular transformation exchanges $w_{2}(X) \leftrightarrow-w_{2}(E)$. It is more useful to work in the basis $\langle F, B\rangle$ for $H^{2}(X)$, so we now have a sum over Chern classes:

$$
\lambda=n B+\left(m-\frac{1}{2}\right) F, \quad n, m \in \mathbb{Z} .
$$

The gauge action now becomes:

$$
\begin{aligned}
& \exp \left[-i \pi \bar{\tau} \lambda_{+}^{2}-i \pi \tau \lambda_{-}^{2}\right]= \\
& \exp \left[-\pi y\left[n^{2} \cosh 2 \theta+2\left(m-\frac{1}{2}\right)^{2} e^{-2 \theta}\right]-i \pi x\left[n^{2}-2 n\left(m-\frac{1}{2}\right)\right]\right]
\end{aligned}
$$

The metric dependence is as in (5.8) with $h \rightarrow h_{M}$ etc. For $n \neq 0$, only a finite number of terms contribute to the integral and the argument is identical to that used for the cusp at $\infty$. However, for $n=0$ the entire sum on $m$ survives the projection by $\int d x$. Notice that in this dual way of writing the theta function, the pointwise vanishing as $\epsilon \rightarrow 0$ comes not because each term in the sum vanishes, but because once we set $n$ to zero, the sum over $m$ is strongly oscillatory. In fact, the sum on $m$ is a derivative of $\vartheta_{1}$ and we can use the estimate:

$$
\sum_{m \in \mathbb{Z}} e^{-2 \pi y \epsilon^{2}\left(m-\frac{1}{2}\right)^{2}} e^{i \pi\left(m-\frac{1}{2}\right)}\left(m-\frac{1}{2}\right)^{k} \sim \text { const } \cdot\left(\frac{1}{\epsilon^{2} y}\right)^{k+1 / 2} e^{-\pi /\left(8 \epsilon^{2} y\right)} .
$$


(The constant vanishes for $k$ even.)

Working at fixed order in $S$, the integral after the projection $\int d x$ becomes a finite sum of terms of the form:

$$
\begin{aligned}
\int_{1}^{\infty} \frac{d y}{y^{3 / 2}}[\widetilde{\mu}(\tau)]_{q^{0}} \sum_{m \in \mathbb{Z}} e^{-2 \pi y \epsilon^{2}\left(m-\frac{1}{2}\right)^{2}} e^{i \pi\left(m-\frac{1}{2}\right)}\left(\frac{(S \cdot F)^{2}}{y \epsilon^{2}}\right)^{t_{1}} \\
\left((S \cdot F)\left(m-\frac{1}{2}\right)\right)^{t_{2}} \cdot\left[K_{1}(S \cdot F) / \epsilon+K_{2} y\left(m-\frac{1}{2}\right) \epsilon\right] .
\end{aligned}
$$

Here $t_{1}, t_{2}$ are nonnegative integers, $\widetilde{\mu}(\tau)$ is a certain modular form, and $K_{1}, K_{2}$ are constants. But $\widetilde{\mu}(\tau) \sim \vartheta_{2}^{8} /\left(\vartheta_{3} \vartheta_{4}\right)^{4} e^{2 p u_{M}+S^{2} T_{M}} \sim q+\cdots$ actually vanishes at the cusp, so in fact $[\widetilde{\mu}(\tau)]_{q^{0}}$ vanishes. This completes the proof of the vanishing theorem. With the vanishing factor removed, the rest of (5.15) behaves like the integral:

$$
\frac{1}{\epsilon} \int_{1}^{\infty} \frac{d y}{y^{3 / 2}}\left(\frac{1}{\epsilon^{2} y}\right)^{t_{1}}\left(\frac{1}{\epsilon^{2} y}\right)^{t_{2}+\frac{1}{2}} e^{-\pi /\left(8 \epsilon^{2} y\right)} .
$$

Making the change of variables $z=\epsilon^{2} y$ shows that this particular integral is non-zero and finite as $\epsilon \rightarrow 0$.

A similar reasoning applies for other rational ruled surfaces. A dangerous looking factor $\vartheta_{2}^{\sigma} \sim q_{D}^{\sigma / 8}$ coming from the $A^{\chi} B^{\sigma}$ measure factor, which can be singular if $\sigma$ is negative enough (and is responsible for SW wall-crossing) is canceled by a vanishing of the theta function near $q_{D}=0$.

In the somewhat analogous case that $X$ is an elliptic surface of $b_{2}^{+}=$ 1 , which maps to a two-dimensional base (necessarily of genus zero) with generic fiber $F$ of genus one, a slightly different situation holds. In a chamber with nearly zero area for $F$ and with a bundle such that $\left(w_{2}(E), F\right) \neq 0$, the Donaldson invariants do not vanish but obey a simple type condition [18] (the SW invariants are likewise not zero). The $u$-plane integrals hence must obey a simple type condition. In fact, an analysis as above shows a pointwise vanishing of the $u$-plane integrand as the area of $F$ goes to zero, but study of the behavior near $u= \pm 1$ shows a surviving contribution from that region, as a result of which the $u$-plane integral does not vanish. Indeed, for $b_{2}^{-}=9$, the factor $\left[\widetilde{\mu}_{q^{0}}=1+\mathcal{O}(q)\right.$ in (5.15) and since (5.16) is nonzero, there can indeed be nonzero contributions. However, acting on $Z_{u}$ by the operator $\left[\frac{\partial^{2}}{\partial p^{2}}-4\right]$, relevant to the simple type condition, is equivalent to an insertion of

$$
4\left(u^{2}-1\right)=\frac{\vartheta_{4}^{8}}{\left(\vartheta_{2} \vartheta_{3}\right)^{2}}
$$

in the integral. This factor increases the order of the zero in $\widetilde{\mu}$ by one and hence, the integral for $b_{2}^{-}=9$ obeys a simple type condition, in accordance with [18]. 


\section{The Blow-Up Formula}

The blow-up formula compares the Donaldson invariants of a four-manifold $X$ to those of a four-manifold $\widehat{X}$ that is obtained by blowing up a point in $X$. Let $\pi: \hat{X} \rightarrow X$ be the blow-down map. Let $b$ be the exceptional curve contracted by $\pi, I(b)$ the corresponding two-observable, and $t$ a complex number. In the blow-up formula, one seeks to compute

$$
\langle\exp (2 p u+I(S)+t I(b))\rangle_{\widehat{\xi}, \widehat{X}},
$$

in a limit in which the area of $b$ is small, in terms of

$$
\langle\exp (2 p u+I(S))\rangle_{\xi, X}
$$

In trying to do so, one assumes that $\widehat{\xi}$ is a class that coincides with $\pi^{*}(\xi)$ away from $b$. The last condition means that $\widehat{\xi}=\pi^{*}(\xi)+j b$ for $j=0$ or 1 . Also, we are identifying a surface $S$ in $X$ (which we can assume does not pass through the point that is to be blown up) with its pullback to $\widehat{X}$.

Let us first discuss on very general grounds why a blow-up formula exists and what its general form would be. We scale up the metric of $X$ by $g \rightarrow t^{2} g$, with very large $t$. Then we blow up a point in $X$, producing an "impurity" that is supposed to be very small, since in the blow-up formula the area of $b$ is supposed to be small. To a distant observer, it must be possible to simulate the effect of the impurity by some local, $\overline{\mathcal{Q}}$-invariant observable. But in the twisted $\mathcal{N}=2$ theory, any local $\overline{\mathcal{Q}}$-invariant observable (supported at a point as opposed to the $k$-form descendants) is a holomorphic function of $u$. There must thus be holomorphic functions $F_{j}(u, t)$, for $j=0,1$, such that

$$
\langle\exp (2 p u+I(S)+t I(b))\rangle_{\widehat{\xi}, \widehat{X}}=\left\langle\exp \left(2 p u+I(S)+F_{j}(u, t)\right)\right\rangle_{\xi, X} .
$$

Thus, the blow-up formula is very similar to the replacement in conformal field theory of a disk or handle by a sum of local operators. The blow-up formula replaces a small region in $X$ which has been modified by the blow-up to produce $\widehat{X}$ by a local operator on $X$.

By applying the same sort of reasoning to the $u$-plane integrals, which simply measure the contributions of certain vacua to the correlation functions, we see that the $u$-plane integrals should obey a formula of the same structure. Moreover, for reasons given in the introduction, the functions appearing in the blow-up formula on the $u$-plane are precisely the functions appearing in the blow-up formula for the Donaldson invariants.

Actually computing the functions $F_{j}$ amounts to comparing the $u$-plane integrands for $X$ with those for $\widehat{X}$. Let $B$ denote the cohomology class dual to $b$. We work in a chamber $B_{+}=0$ (more precisely, for any given correlator, 
in a chamber $B_{+}<\epsilon$ for sufficiently small $\epsilon$ ). Take $\widetilde{S}=S+t b$ and substitute into the theta function (3.18) and use the condition $B_{+}=0$ to obtain:

$$
\Psi_{\widehat{X}}=\Psi_{X} \exp \left[\frac{\pi t^{2}}{8 \pi y h^{2}}\right] \sum_{n \in \mathbb{Z}+\frac{1}{2} w_{2}(\tilde{E}) \cdot B} \exp \left[i \pi \tau n^{2}+i n t / h\right] e^{-i \pi n}
$$

Similarly, the measure factor for the blown up manifold is related to that of the original manifold by:

$$
\widehat{f}_{\widehat{X}}=\widehat{f}_{X} \vartheta_{4}^{-1} \exp \left[-t^{2} \widehat{T}(u)\right]
$$

The $\vartheta_{4}^{-1}$ factor comes because the blow-up changes $\chi$ and $\sigma$, and the other factor comes because $\widetilde{S}^{2}=S^{2}-t^{2}$. Now, taking $\tau \rightarrow \tau+1$ on this expression (so as to facilitate comparison to [10], where a subgroup of $S L(2, \mathbb{Z})$ that differs from $\Gamma^{0}(4)$ by conjugation by $\tau \rightarrow \tau+1$ is used), we see that the blowup has the effect of modifying the integrand in a way which is equivalent to the substitution

$$
e^{2 p u_{(\infty, 1)}} \rightarrow e^{2 p u_{(\infty, 1)}} \exp \left[\frac{t^{2}}{24}\left(\frac{E_{2}}{\left(h_{(\infty, 1)}\right)^{2}}-8 u_{(\infty, 1)}\right)\right] \frac{\vartheta_{3}\left(\frac{t}{2 \pi h_{(\infty, 1)}} \mid \tau\right)}{\vartheta_{3}(0 \mid \tau)}
$$

in the case where $w_{2}(\widetilde{E}) \cdot B=0 \bmod 2$. Likewise, it is equivalent to the substitution

$$
e^{2 p u_{(\infty, 1)}} \rightarrow-\kappa e^{2 p u_{(\infty, 1)}} \exp \left[\frac{t^{2}}{24}\left(\frac{E_{2}}{\left(h_{(\infty, 1)}\right)^{2}}-8 u_{(\infty, 1)}\right)\right] \frac{\vartheta_{1}\left(\frac{t}{2 \pi h_{(\infty, 1)}} \mid \tau\right)}{\vartheta_{3}(0 \mid \tau)}
$$

when $w_{2}(\widetilde{E}) \cdot B=1 \bmod 2$. The equations (6.6), (6.7) are equivalent to the expressions in [10], eqs. 4.5.1, 4.5.2. (Note there is a misprint in these formulae; they should have $\left.G(\tau) / f^{2}(\tau)\right)$.

In order to interpret (6.6) and (6.7), it is helpful to expand the expressions in powers of $t$ and then re-express the modular functions of $\tau$ as power series in the function $u_{(\infty, 1)}$. The resulting expression is a power series in $t$ whose coefficients are polynomials in $u_{(\infty, 1)}$. For example, when $w_{2}(\widetilde{E}) \cdot B=$ $1 \bmod 2$ we have

$$
(-\kappa) \exp \left[\frac{t^{2}}{24}\left(\frac{E_{2}}{\left(h_{(\infty, 1)}\right)^{2}}-8 u_{(\infty, 1)}\right] \frac{\vartheta_{1}\left(\frac{t}{2 \pi h_{(\infty, 1)}} \mid \tau\right)}{\vartheta_{3}(0 \mid \tau)}=\sum_{k \geq 1} t^{k} \mathcal{B}_{k}\left(u_{(\infty, 1)}\right)\right.
$$


where (as we will see shortly) $\mathcal{B}_{k}$ is a polynomial. Since $u_{(\infty, 1)}$ multiplies $p$ in the integral representation we arrive at the relation between invariants:

$$
\begin{aligned}
& \langle\exp [I(S)+t I(B)+p \mathcal{O}]\rangle_{\widehat{X}, \widehat{w_{2}(E)}=w_{2}(E)+B}= \\
& \sum_{k \geq 1} t^{k} \mathcal{B}_{k}\left(\frac{\partial}{\partial p}\right) \Phi_{X, w_{2}(E)}(S, p)=\sum_{k \geq 1} t^{k}\left\langle\exp [I(S)+p \mathcal{O}] \mathcal{B}_{k}(\mathcal{O})\right\rangle_{X, w_{2}(E)}
\end{aligned}
$$

We will now determine the polynomials $\mathcal{B}_{k}$. We use the formula:

$$
\frac{\vartheta_{1}(z \mid \tau)}{2 \pi \eta^{3}(\tau)}=-z \exp \left[-\sum_{k=1}^{\infty} \frac{G_{2 k}(\tau)}{2 k} z^{2 k}\right]
$$

where $G_{2 k}=2 \zeta(2 k) E_{2 k}$ are Eisenstein functions, and $E_{2 k}$ are normalized Eisenstein series of weight $2 k$. Using $\vartheta_{1}^{\prime}(0 \mid \tau)=-2 \pi \eta^{3}=-\pi \vartheta_{2} \vartheta_{3} \vartheta_{4}$ we rewrite (6.8) as:

$$
\sum_{k \geq 1} t^{k} \mathcal{B}_{k}\left(u_{(\infty, 1)}\right)=t \exp \left[-\frac{t^{2}}{3} u_{(\infty, 1)}-\sum_{k=2}^{\infty} \frac{t^{2 k}}{2 k} \frac{G_{2 k}(\tau)}{\left(2 \pi h_{(\infty, 1)}\right)^{2 k}}\right] .
$$

Now we note that the Eisenstein functions $G_{2 k}$ can be expressed as:

$$
G_{2 k}=\sum_{4 s+6 t=2 k} c_{k, s, t}\left(G_{4}\right)^{s}\left(G_{6}\right)^{t}
$$

where $c_{k, s, t}$ are rational numbers. Using the expression for Eisenstein series in terms of theta functions, we now show:

$$
\begin{aligned}
& \frac{G_{4}}{\left(2 \pi h_{(\infty, 1)}\right)^{4}}=\frac{1}{45}\left(4 u_{(\infty, 1)}^{2}-3\right), \\
& \frac{G_{6}}{\left(2 \pi h_{(\infty, 1)}\right)^{6}}=\frac{2}{945}\left(8 u_{(\infty, 1)}^{3}-9 u_{(\infty, 1)}\right),
\end{aligned}
$$

and hence the $\mathcal{B}_{k}$ are polynomials in $u_{(\infty, 1)}$ with rational coefficients. The ratio of $\vartheta$ functions can be expressed in terms of Weierstrass $\sigma$ functions and this is the form in which the blow-up formula was originally stated, e.g. in [19].

As a simple example of (6.9), we may consider the first term in the expansion:

$$
\mathcal{D} \widehat{\widehat{X}} \frac{w_{2}(E)}{=w_{2}(E)+B}\left(S^{n}, p^{m}, B\right)=\mathcal{D}_{w_{2}(E)}^{X}\left(S^{n}, p^{m}\right) .
$$


For the other case one must expand (6.6) to order 4. One finds:

$$
\mathcal{D}_{\widehat{w_{2}(E)}=w_{2}(E)}^{\widehat{X}}\left(S^{n}, p^{m}, B^{4}\right)=-2 \mathcal{D}_{w_{2}(E)}^{X}\left(S^{n}, p^{m}\right) .
$$

The factor of 2 in this formula is the reason that in Donaldson theory, invariants outside the so-called "stable range" are not integral but have factors of $\frac{1}{2}$.

\section{Universal Form of SW Contributions}

We now analyze the SW contributions to $Z_{D}$, that is, the contributions of the special vacua at $u= \pm 1$. First, we outline the basic mechanism by which the contributions of the $u= \pm 1$ vacua are computed in terms of SW invariants. Then we obtain precise formulas, without assuming a simple type condition, using the $u$-plane wall crossing formulas of section 4 to determine some universal functions that will be required.

Near $u=1$, there is a distinguished special coordinate on the $u$-plane, namely $a_{D}$. It is part of a vector multiplet that also contains a distinguished photon $A_{D}$, and fermions $\eta_{D}, \psi_{D}, \chi_{D}$ (which in the twisted theory are a zeroform, a one-form, and a self-dual two-form). The theory near $u=1$ has a $U(1)_{R}$ symmetry (violated quantum mechanically by an anomaly involving the dimension of SW moduli space) under which $A_{D}$ is invariant and $a_{D}$ has $R$ charge 2 .

Because there is a distinguished special coordinate near $u=1$ and no issue of duality symmetry, the theory near $u=1$ can be analyzed as a topological quantum field theory of a standard sort. By picking a suitable functional $V$ and adding to the Lagrangian a term $\lambda\{\overline{\mathcal{Q}}, V\}$ with $\lambda \rightarrow \infty$, one localizes on supersymmetric configurations (solutions of the SW equations) which must be counted, in a suitable way, to give the correlation functions. We have explained in section 2.3 that duality presents an obstruction to such a treatment of the $u$-plane contribution.

The theory at $u=1$ has, in addition to the vector multiplet, a massless hypermultiplet whose bosonic part will be called $M$. In the topologically twisted theory on a four-manifold $X$, for reasons explained in section 4.2 of [33], the dual $U(1)$ gauge theory with connection $A_{D}$ involves not quite a connection on a line bundle but a $\operatorname{Spin}^{c}$ connection. For our purposes, we symbolically associate a $\operatorname{Spin}^{c}$ structure with a bundle $S_{+} \otimes L$, where $S_{+}$is the positive spin bundle and $L^{2}$ is a line bundle such that $c_{1}\left(L^{2}\right)$ is congruent to $w_{2}(X)$ modulo 2 . (The factors $S_{+}$and $L$ in $S_{+} \otimes L$ do not really exist separately.) We will use the symbol $\lambda$ to denote $\frac{1}{2} c_{1}\left(L^{2}\right)$, regarded as an element of $\frac{1}{2} \Gamma$ (as before $\Gamma=H^{2}(X, \mathbb{Z})$ ) that is congruent to $\frac{1}{2} w_{2}(X)$ modulo 1. We call $\lambda$ the first Chern class of the $\operatorname{Spin}^{c}$ structure, and we identify a 
$\operatorname{Spin}^{c}$ structure with its first Chern class. To avoid cluttering the formulas, we will write in this section simply $F$, instead of $F_{D}$, for the curvature of $A_{D}$, and $\lambda$, instead of $\lambda_{D}$, for the first Chern class of the $\operatorname{Spin}^{c}$ structure; likewise we will omit the subscript $D$ for the fermions $\eta, \psi, \chi$. In the twisted theory, the bosonic part of the charged hypermultiplet is a section $M$ of the $\operatorname{Spin}^{c}$ bundle $S_{+} \otimes L$.

The supersymmetric configurations on which the contribution at $u=1$ is localized can be described as follows. The supermultiplet that contains $a_{D}$ and $A_{D}$ also contains, along with the fermions $\eta, \psi, \chi$, the familiar auxiliary field $D$. In the presence of the hypermultiplet, the equation of motion for $D$ (keeping only the bosonic terms) says that $D$ is equal to the hyper-Kahler moment map of the hypermultiplets; we write this as $D=(\bar{M} M)_{+}$. The supersymmetry transformation law of $\chi$ is $\delta \chi=i\left(F_{+}-D\right)$, or, with $D$ integrated out, $\delta \chi=i\left(F_{+}-(\bar{M} M)_{+}\right)$. So for a bosonic configuration to be supersymmetric, it must satisfy

$$
F_{+}=(\bar{M} M)_{+} .
$$

This is one of the SW equations. The other SW equation, which is the Dirac equation

$$
\not D M=0,
$$

arises because the hypermultiplet contains a fermi field $\zeta$ whose $\overline{\mathcal{Q}}$-variation is $\delta \zeta=\not D M$. So supersymmetric configurations are simply solutions of the SW equations. Let $\mathcal{M}_{\lambda}$ be the moduli space of SW solutions of given $\lambda$.

The dimension of $\mathcal{M}_{\lambda}$ is (according to an index theorem)

$$
d_{\lambda}=-\frac{2 \chi+3 \sigma}{4}+\lambda^{2}
$$

In the special case that $b_{1}=0, b_{2}^{+}=1$, this amounts to

$$
d_{\lambda}=-2-\frac{\sigma}{4}+\lambda^{2} .
$$

The basic topological observable in the $u=1$ theory is simply the zeroform operator $\mathcal{O}^{(0)}=a_{D}$, which has $R$ charge two and so is associated topologically with a two-dimensional class on $\mathcal{M}_{\lambda}$. It has a one-form descendant, which is simply $\psi$, and a two-form descendant, which is simply $F$. The correlation functions of the two-form descendant hence measure simply the first Chern class $\lambda$, and the one-form descendant of course does not enter if $b_{1}(X)=0$. In that case, therefore, the only relevant quantum topological 
observable is $a_{D}$ itself. If $d_{\lambda}=2 n$, the SW invariant is defined as ${ }^{16}$

$$
S W(\lambda)=\left\langle a_{D}^{n}\right\rangle_{\lambda}=\int_{\mathcal{M}_{\lambda}} a_{D}^{n} .
$$

(In all known cases with $b_{2}^{+}>1, S W(\lambda)$ is non-zero only for $\lambda$ such that $n=0$. But all values of $n$ contribute for $b_{2}^{+}=1$. This is a consequence of the fact that, for sufficiently negative $\sigma$, wall crossing can occur for $\lambda$ with arbitrarily large $n$.)

When we speak of the SW contribution to Donaldson invariants from $u=1$, we mean by definition the contribution of SW solutions with $M \neq 0$. Solutions with $M=0$ can be "confused" with the continuous contribution of the $u$-plane. The restriction to solutions with $M \neq 0$ is, except for $b_{2}^{+} \leq 1$, a mild one in the following sense. A solution with $M=0$ is an abelian instanton, and is possible only if $\lambda_{+}=0$. In this case $2 \lambda$ is an integral and anti-self-dual cohomology class. For $b_{2}^{+}>0$, there is no such class for a generic metric, ${ }^{17}$ so the SW contributions with $M \neq 0$ and the continuous contribution of the $u$-plane are well-separated. However, for $b_{2}^{+}=1$, the condition that a given $\lambda$ obeys $\lambda_{+}=0$ puts only one condition on the metric. Hence in a generic one-parameter family of metrics, one may "cross" a metric for which $\lambda_{+}=0$. It is known that $S W(\lambda)$ jumps by \pm 1 in crossing such a wall. Such wall-crossing does not occur for $b_{2}^{+}>1$, because in that case $\lambda_{+}=0$ puts more than one condition on the metric, so that in a generic one-parameter family of metrics, the SW and $u$-plane contributions never meet.

The intuitive picture of SW wall crossing that we want to justify is that in crossing a wall, some SW solutions move to (or from) $M=0$, and their contributions to the Donaldson invariants disappear from the $u=1$ vacuum and move onto the $u$ plane. Thus, the jumping of the SW invariant in crossing a wall, if suitably measured, will just cancel the $u=1$ part of the wall-crossing formula of the $u$-plane integral. A full justification of this will occupy the rest of this section, but let us first make the following simple remarks. For $d_{\lambda}<0, \mathcal{M}_{\lambda}$ is generically empty and the SW invariant vanishes. For $M$ to go to zero in an SW solution requires, as we saw above, $\lambda_{+}=0$.

\footnotetext{
${ }^{16}$ Mathematically, there is a tautological $U(1)$ bundle over $\mathcal{M}_{\lambda}$ defined by dividing the space of solutions to the SW equations using only based gauge transformations. $a_{D}$ is then, up to a normalization, the first Chern class of this $U(1)$ bundle.

${ }^{17}$ Except $\lambda=0$. On the $u$-plane, the computation in section 4 shows that, at least in the simply-connected case, there is never wall-crossing associated with $\lambda=0$; the wallcrossing came from a term $(\lambda, \omega) / y^{1 / 2}$ that vanishes for $\lambda=0$. In SW theory, $\lambda=0$ never contributes for a simply-connected four-manifold $X$ with $b_{2}^{+}=1$. In fact, $\lambda=0$ is only possible if $X$ is spin, in which case for $b_{2}^{+}=1$, a theorem of Donaldson shows that $b_{2}^{-}=1$ and the intersection form is that of $\mathbb{P}^{1} \times \mathbb{P}^{1}$. But for $b_{1}=0, b_{2}^{+}=b_{2}^{-}=1$, and $\lambda=0$, the virtual dimension of the SW moduli space is negative, so $S W(\lambda=0)=0$.
} 
So $4 \lambda^{2}$ is a negative integer with

$$
4 \lambda^{2}-(8+\sigma) \geq 0
$$

These then are the standard conditions for SW wall-crossing. They also are exactly the conditions (4.11) we found in section 4 for a contribution to $u$-plane wall-crossing at $u= \pm 1$. This is a first indication that SW wall-crossing and the $u= \pm 1$ wall-crossing of the $u$-plane integrals can be matched up.

Effective Couplings Of The $u=1$ Theory

The Lagrangian of the twisted theory near $u=1$ has the form

$$
L=\{\overline{\mathcal{Q}}, W\}+\int_{X}(c(u) F \wedge F+p(u) \operatorname{Tr} R \wedge R+\ell(u) \operatorname{Tr} R \wedge \widetilde{R}+\cdots)
$$

In fact, the most general $\overline{\mathcal{Q}}$-invariant Lagrangian that can be constructed with the multiplets in question takes this form, with some $W$ and some holomorphic functions $c, p$, and $\ell$. The terms involving $c, p$, and $\ell$ are close cousins of terms studied in section 2 on the $u$-plane. The $c(u) F \wedge F$ term is the fourth descendant of a zero-form observable $\mathcal{O}^{(0)}=\widetilde{\mathcal{F}}_{M}(u)$ which is quite analogous to the prepotential $\mathcal{F}$ of equation (2.14). (To be more precise, this descendant contains additional terms involving $\psi$ which do not contribute for $b_{1}==0$ and are omitted in (7.7).) In fact, one can think of $\widetilde{\mathcal{F}}_{M}(u)$ as the prepotential of the effective theory of dual photons and monopoles near $u=1$. In the other terms, $\operatorname{Tr} R \wedge R$ and $\operatorname{Tr} R \wedge \widetilde{R}$ are differential forms whose integrals are multiples of the signature $\sigma$ and Euler characteristic $\chi$ of $X$. Upon exponentiation in the path integral, these interactions give factors of the general form $A^{\chi} B^{\sigma}$ familiar from section 2 .

The three couplings indicated explicitly in (7.7) give rise in the path integral for a given $\operatorname{Spin}^{c}$ structure $\lambda$ to factors

$$
C(u)^{\lambda^{2} / 2} P(u)^{\sigma / 8} L(u)^{\chi / 4}
$$

where the functions $C, P$, and $L$ are essentially exponentials of $c, p$, and $\ell$. We have seen such factors before; the first corresponds to a factor in the lattice theta function on the $u$-plane, and the other two are clearly analogous to the functions in equations (2.16) and (3.2).

One might ask why the functions $c, p$, and $\ell$, or $C, P$, and $L$, do not precisely equal the analogous functions in the $u$-plane calculation. The answer is that in the $u$-plane analysis, one deals with an effective action in which the monopole hypermultiplet has been integrated out, while in the present discussion we are using an effective Lagrangian that includes the monopole 
fields. In particular, the functions $C, P$, and $L$ should be completely nonsingular (and non-zero) at $u=1$, as the singularities in the $u$-plane description come from integrating out the massless hypermultiplet. It is quite conceivable that there is a simple recipe to relate the couplings with the hypermultiplets present to couplings with hypermultiplets integrated out, but in the present paper we will simply determine the functions $C, P$, and $L$ by comparison to the wall-crossing formula.

An analogous issue arises in the mapping from the microscopic observable $\exp (p \mathcal{O}+I(S))$ to an effective interaction in the theory at $u=1$. The general considerations are as in the discussion on the $u$-plane. The microscopic operator $\exp (p \mathcal{O})$ simply maps to $\exp (2 p u)$ in the $u$-plane description. The operator $I(S)$ maps to $\int_{S}\left(\frac{i}{4 \pi} \frac{d u}{d a_{D}}\left(F_{-}+D_{+}\right)-\frac{i}{32 \sqrt{2} \pi} \frac{d^{2} u}{d a_{D}^{2}} \psi \wedge \psi\right)$. This computation is in fact precisely as it was on the $u$-plane, except now using the vector multiplet that contains $a_{D}, A_{D}$. Because of the existence of a fermi field $\chi$ with $\delta \chi=i\left(F_{+}-D_{+}\right)$and the fact that the $u=1$ computation is done by localization on supersymmetric fields, we actually can replace $D_{+}$here by $F_{+}$, so that $I(S)$ maps to the familiar topological field theory expression $\int_{S}\left(\frac{i}{4 \pi} \frac{d u}{d a_{D}} F-\frac{i}{32 \sqrt{2} \pi} \frac{d^{2} u}{d a_{D}^{2}} \psi \wedge \psi\right)$; also, the terms involving $\psi$ can be dropped if $b_{1}(X)=0$. In reducing $\exp (I(S))$ to the $u=1$ description, there is a contact term, governed by the same general logic that applied on the $u$-plane. So we get (assuming $b_{1}(X)=0$ )

$$
\exp (p \mathcal{O}+I(S)) \rightarrow \exp \left(2 p u+\frac{i}{4 \pi} \int_{S} \frac{d u}{d a_{D}} F+S^{2} T^{*}(u)\right) .
$$

A priori the function $T^{*}(u)$ might differ from the analogous function $T(u)$ on the $u$-plane, since $T(u)$ is obtained after integrating out the hypermultiplet. However, we will see that $T^{*}=T$ - this particular coupling is unchanged by integrating out the monopoles. (Therefore $T$ has no singularity at $u=1$ or -1 , an assumption that was made in determining $T$.)

\section{Form of the Path Integral}

We want to analyze the contribution from the monopole vacuum at $u=1$ to the path integral that defines the generating function $\left\langle e^{p \mathcal{O}+I(S)}\right\rangle_{\xi}$ (where as in the introduction, we sum over all classes of $S O(3)$ bundles $E$ of fixed $\left.\xi=w_{2}(E)\right)$. The path integral in this vacuum includes a sum over $\operatorname{Spin}^{c}$ structures $\lambda$, that is over the "line bundle" of the dual photon $A_{D}$. We will write $\left\langle e^{p \mathcal{O}+I(S)}\right\rangle_{\xi, u=1}$ for the contribution of the $u=1$ vacuum to the generating function, and $\left\langle e^{p \mathcal{O}+I(S)}\right\rangle_{\xi, 1, \lambda}$ for the contribution in the $u=1$ vacuum from a particular $\operatorname{Spin}^{c}$ structure $\lambda$. Thus

$$
\left\langle e^{p \mathcal{O}+I(S)}\right\rangle_{\xi, u=1}=\sum_{\lambda}\left\langle e^{p \mathcal{O}+I(S)}\right\rangle_{\xi, 1, \lambda}
$$


Our goal here is to work out a formula for $\left\langle e^{p \mathcal{O}+I(S)}\right\rangle_{\xi, 1, \lambda}$ in terms of the invariant $S W(\lambda)$.

The requisite path integral has several factors. We must use the formula (7.9) by which $e^{p \mathcal{O}+I(S)}$ is translated to an effective operator in the $u=1$ vacuum, the factors in (7.8), and two additional factors. The first is a factor of 2, because in defining the Donaldson invariants (and in particular $\left.\left\langle e^{p \mathcal{O}+I(S)}\right\rangle_{\xi}\right)$, it is not customary to divide by the order of the center of $S U(2)$. Also, upon making the duality transformation to the natural description at $u=1$, one gets an analog of the phase factor (3.11). This phase factor is $e^{2 i \pi\left(\lambda_{0} \cdot \lambda+\lambda_{0}^{2}\right)}$, where $\lambda_{0}$ is the fixed element of $\frac{1}{2} w_{2}(E)+\Gamma$ that entered in (3.11). (A path integral explanation of the $\lambda$ dependence of this factor is in [33].)

After multiplying these factors together, we get a function of $a_{D}$ that must be integrated over $\mathcal{M}_{\lambda}$ to get the contribution of the $\operatorname{Spin}^{c}$ structure $\lambda$ to the Donaldson invariants. So we have

$$
\begin{aligned}
\left\langle e^{p \mathcal{O}+I(S)}\right\rangle_{\xi, 1, \lambda}=\int_{\mathcal{M}_{\lambda}} 2 e^{2 i \pi\left(\lambda_{0} \cdot \lambda+\lambda_{0}^{2}\right)} \exp & \left(2 p u+\frac{i}{4 \pi} \int_{S} \frac{d u}{d a_{D}} F+S^{2} T^{*}(u)\right) \\
\cdot C(u)^{\lambda^{2} / 2} P(u)^{\sigma / 8} L(u)^{\chi / 4} \cdot & (7.11)
\end{aligned}
$$

What it means to integrate over $\mathcal{M}_{\lambda}$ is that one must expand in powers of $a_{D}$ - which represents a two-dimensional cohomology class on $\mathcal{M}_{\lambda}-$ near $a_{D}=$ 0 , and extract the coefficient of $a_{D}^{n}$, where $n=d_{\lambda} / 2=-(2 \chi+3 \sigma) / 8+\lambda^{2} / 2$. Then we integrate over $\mathcal{M}_{\lambda}$, using $\int_{\mathcal{M}_{\lambda}} a_{D}^{n}=S W(\lambda)$. We can write this as a residue,

$$
\begin{aligned}
& \left\langle e^{p \mathcal{O}+I(S)}\right\rangle_{\xi, 1, \lambda}=S W(\lambda) \cdot \operatorname{Res}_{a_{D}=0} \frac{d a_{D}}{a_{D}^{1-(2 \chi+3 \sigma) / 8+\lambda^{2} / 2}} \cdot 2 e^{2 i \pi\left(\lambda_{0} \cdot \lambda+\lambda_{0}^{2}\right)} \\
& \cdot \exp \left(2 p u+\frac{i}{4 \pi} \int_{S} \frac{d u}{d a_{D}} F+S^{2} T^{*}(u)\right) \cdot C(u)^{\lambda^{2} / 2} P(u)^{\sigma / 8} L(u)^{\chi / 4}
\end{aligned}
$$

Note that we have not been careful to normalize the operator $a_{D}$ topologically; it is not necessary to do so as a change in the normalization could be absorbed in a rescaling of the yet-undetermined functions $C, P$, and $L$.

\section{Determination of $C, P$, and $L$}

To determine those factors, we will compare to the wall crossing formulas in the special case $b_{2}^{+}=1$, that is $\chi+\sigma=4$. Assuming that $\lambda$ is such that $n \geq 0$ (otherwise (7.12) vanishes), SW wall crossing occurs at walls at which $\lambda_{+}=0$. It is known topologically that at such a wall, $S W(\lambda)$ changes by \pm 1 (when $\left.b_{1}=0\right)$. The sign depends on the direction in which one crosses the wall, and it will not be necessary to keep track of it in order to determine the unknown functions. 
The change in (7.12) is hence

$$
\begin{aligned}
& \Delta\left\langle e^{p \mathcal{O}+I(S)}\right\rangle_{\xi, 1, \lambda}= \pm \operatorname{Res}_{a_{D}=0} \frac{d a_{D}}{a_{D}^{-\sigma / 8+\lambda^{2} / 2}} \cdot 2 e^{2 i \pi\left(\lambda_{0} \cdot \lambda+\lambda_{0}^{2}\right)} \\
& \cdot \exp \left(2 p u+\frac{i}{4 \pi} \int_{S} \frac{d u}{d a_{D}} F+S^{2} T^{*}(u)\right) \cdot C(u)^{\lambda^{2} / 2} P(u)^{\sigma / 8} L(u)^{1-\sigma / 4} .
\end{aligned}
$$

On the other hand, the contribution of $u=1$ to the wall-crossing formula for the $u$-plane integral was given in equation (4.8). In order to compare these formulae note first that $d a_{D} / d u=-h_{M}\left(\tau_{D}\right)=\frac{i}{2} \vartheta_{3} \vartheta_{4}\left(\tau_{D}\right){ }^{18}$ Similarly we must have $T^{*}(u)=T(u)$, since the $q_{D}$ expansion of $T(u)$ around the monopole cusp $\tau=0$ is exactly $T_{M}\left(q_{D}\right)$ defined in (4.9). ${ }^{19}$ In a similar way we find that the unknown functions $C, P$, and $L$ can be uniquely determined in order for the formulas to agree:

$$
\begin{aligned}
& C=\frac{a_{D}}{q_{D}}, \\
& P=-\frac{4 \vartheta_{2}\left(\tau_{D}\right)^{8}}{h_{M}^{4}} a_{D}^{-1}, \\
& L=-\frac{2 i}{h_{M}^{2}},
\end{aligned}
$$

where we have used the identity:

$$
q_{D} \frac{d a_{D}}{d q_{D}}=\frac{1}{16 i} \frac{\vartheta_{2}^{8}}{\vartheta_{3} \vartheta_{4}} .
$$

In particular, the prepotential of the theory is obtained from

$$
\frac{d^{2}}{d a_{D}^{2}} \widetilde{\mathcal{F}}_{M}\left(a_{D}\right)=\tau_{D}-\frac{1}{2 \pi i} \log a_{D} .
$$

Substituting (7.14) in (7.12) we get a formula for the contribution of a $\operatorname{Spin}^{c}$ structure $\lambda$ at $u=1$ to the Donaldson invariants:

$$
\begin{aligned}
&\left\langle e^{p \mathcal{O}+I(S)}\right\rangle_{\xi, 1, \lambda}=\frac{S W(\lambda)}{16} \cdot e^{2 i \pi\left(\lambda_{0} \cdot \lambda+\lambda_{0}^{2}\right)} \\
& \cdot \operatorname{Res}_{q_{D}=0}\left[\frac{d q_{D}}{q_{D}} q_{D}^{-\lambda^{2} / 2} \frac{\vartheta_{2}^{8+\sigma}}{a_{D} h_{M}}\left(2 i \frac{a_{D}}{h_{M}^{2}}\right)^{(\chi+\sigma) / 4}\right. \\
&\left.\cdot \exp \left[2 p u_{M}-i(\lambda, S) / h_{M}+S^{2} T_{M}\right]\right] .
\end{aligned}
$$

\footnotetext{
${ }^{18}$ There is a subtle minus sign here related to the fact that $h$ is a form of weight 1 .

${ }^{19}$ If we follow our notation to its logical conclusion then we should speak of a $q_{M}$ expansion and $a_{M}$, etc. However, we do not do this since the notation $a_{D}$ is standard.
} 
In order to complete the result we must say how to expand $a_{D}$ in $q_{D}$. This is a simple exercise in elliptic functions. We know from $[2,3]$ that $a_{D}(u)$ is given by $a_{D}(u)=\left(\frac{1-u}{2 i}\right)_{2} F_{1}\left(\frac{1}{2}, \frac{1}{2} ; 2 ; \frac{1-u}{2}\right)$. This may be expressed in terms of complete elliptic integrals which themselves may be expressed in terms of modular functions. The result is that:

$$
a_{D}=-\frac{i}{6}\left(\frac{2 E_{2}-\vartheta_{3}^{4}-\vartheta_{4}^{4}}{\vartheta_{3} \vartheta_{4}}\right)
$$

as a function of $q_{D}$.

The analogous contribution from $u=-1$ follows almost immediately, as the theory when formulated on flat $\mathbb{R}^{4}$ has a symmetry under $u \leftrightarrow-u$. One need only replace $u$ by $-u$, and $a_{D}$ by $a+a_{D}$ (which is the appropriate local parameter near $u=-1$ ), to get the formula analogous to (7.17) for the contribution of the vacuum at $u=-1$ to the Donaldson invariants. As in eqn. (2.66) of [31], this replacement, apart from $u \rightarrow-u$, introduces a multiplicative factor of $i(\chi+\sigma) / 4-\xi^{2}$ that reflects the fact that the transformation $u \rightarrow-u$, though a symmetry on flat $\mathbb{R}^{4}$, has an anomaly on a four-manifold.

As a simple but important example of the above procedure, one easily recovers equation (2.17) of [4] in the simple type case when $d_{\lambda}=0$. In this case we need only take the leading terms in the $q$-expansions in (7.17). Using $u_{M}=1+\cdots, T_{M}=1 / 2+\cdots, h_{M}=1 /(2 i)+\cdots$ and $a_{D}=16 i q_{D}+\cdots$ we find that (7.17) reduces to

$$
2^{1+\frac{7 \chi}{4}+\frac{11 \sigma}{4}} e^{2 p+S^{2} / 2} e^{2(S, \lambda)} e^{2 i \pi\left(\lambda_{0} \cdot \lambda+\lambda_{0}^{2}\right)} .
$$

Identifying $2 \lambda$ with the variable called $x$ in [4], equation (2.17), we get perfect agreement. ${ }^{20}$

More generally, the above formula for $\left\langle e^{p \mathcal{O}+I(S)}\right\rangle_{\xi, 1, \lambda}$ implies that fourmanifolds of $b_{1}=0, b_{2}^{+}>1$ (for which the $u$-plane integral vanishes, so everything comes from the SW contributions at $u= \pm 1$ ) are of simple type in the generalized sense that $\left(\frac{\partial^{2}}{\partial p^{2}}-4\right)^{r}\left\langle e^{p \mathcal{O}+I(S)}\right\rangle_{\xi, 1, \lambda}=0$ for some $r$. One simply takes $r$ to exceed the maximum value of $n=d_{\lambda} / 2$ that arises for any $\lambda$ with $S W(\lambda) \neq 0$. (There is such a maximum, since it is known that $S W(\lambda)=0$ for all but finitely many $\lambda$.) The formula (7.17) entails an expansion in powers of $(u-1)$ (or $\left.a_{D}\right)$ in which one only "sees" terms of order at most $(u-1)^{n}$, hence

$$
\left(\frac{\partial^{2}}{\partial p^{2}}-4\right)^{r} Z_{D}=0
$$

if $r$ exceeds the maximum possible value of $n$. Hence the manifold is of generalized simple type in the sense formulated by Kronheimer and Mrowka.

\footnotetext{
${ }^{20}$ We recall from the footnote just before eqn. (3.13) that, to agree with the mathematical literature, we have modified the orientation convention of [4] by a factor $(-1)^{\frac{1}{2}\left(w_{2}(E)^{2}+w_{2}(E) \cdot w_{2}(X)\right)}$.
} 


\section{Evaluation in Certain Chambers}

As explained in the introduction, the wall-crossing, blow-up, and vanishing properties of $Z_{u}$ completely determine its value for all $X$ of $b_{1}=0, b_{2}^{+}=1$. However, as explained in the introduction, it is desirable to have a more effective formula.

We derive such a formula here in the case $b_{-}>1$ and $w_{2}(E)=0$. We follow the general calculation of Borcherds [11] (which in turn is an improvement on calculations done in the literature on quantum string corrections, such as $[22,13])$.

We let $I^{p, q}$ be the lattice with quadratic form $\sum_{i=1}^{p} x_{i}^{2}-\sum_{j=1}^{q} y_{j}^{2}$. The lattice $H^{2}(X, \mathbb{Z})$ is isomorphic to $I^{1, b_{2}-1}$. If we let $I I^{1,1}$ be the even unimodular rank two lattice (often called $H$ ) with intersection form $\left(\begin{array}{ll}0 & 1 \\ 1 & 0\end{array}\right)$, then $I^{1, b_{2}-1}$ is isomorphic to $I I^{1,1} \oplus I^{0, b_{2}-2}$. Our computation will depend on a choice of such a decomposition (and for each such decomposition, we will compute the $u$-plane integral in a certain chamber). Fixing such a decomposition, we choose a basis of primitive null vectors $z, z^{\prime}$, with $\left(z, z^{\prime}\right)=1$, which generate the summand $I I^{1,1}$ of $H^{2}(X, \mathbb{Z})$. The second summand $I^{0, b_{2}-1}$ will be called $K . K$ can be identified as

$$
K=z^{\perp} /\langle z\rangle \text {, }
$$

and with this definition depends only on the choice of a primitive null vector $z$ and not on $z^{\prime}$.

An example of the situation considered here is that $X$ is a rational ruled surface, and $z$ is the class of the fiber. The situation we are considering is thus very close to that of section 5 , except that $w_{2}(E)$ is zero.

\subsection{The Lattice $K$ and the Reduction Formula}

Our goal is to reduce the computation of the $u$-plane integrals to one involving theta functions for the lattice $K$.

We let $P_{ \pm}$be the orthogonal projections of $H^{2}(X, \mathbb{Z})$ to self-dual and anti-selfdual parts, and and let $\widetilde{P}_{ \pm}$be the orthogonal projections onto the orthogonal complement of $z_{ \pm}=P_{ \pm}(z)$ within the self-dual and anti-self-dual subspaces of $H^{2}(X, \mathbb{Z})$. In particular, $z_{+}=(z, \omega) \omega$, and $\widetilde{P}_{+}=0$ since the self-dual subspace of $H^{2}(X, \mathbb{Z})$ is one-dimensional, generated by $z_{+}$. We have

$$
\begin{aligned}
& \lambda_{+}=\frac{\left(\lambda_{+}, z_{+}\right)}{z_{+}^{2}} z_{+}=(\lambda, \omega) \omega, \\
& \lambda_{-}=\frac{\left(\lambda_{-}, z_{-}\right)}{z_{-}^{2}} z_{-}+\widetilde{P}_{-}(\lambda),
\end{aligned}
$$


and the second line is an orthogonal sum in $\mathbb{R}^{0, b_{-}}:\left(z_{-}, \widetilde{P}_{-}(\lambda)\right)=0$.

The reduction formula is now obtained by writing

$$
\lambda=\lambda^{K}+c z^{\prime}+n z,
$$

with $c, n \in \mathbb{Z}$ and $\lambda^{K} \in K$, and then doing a Poisson summation formula on $n$. Using the isomorphism $H^{2}(X, \mathbb{Z}) \cong I^{1, b_{-}} \cong I I^{1,1} \oplus I^{0, b_{-}-1}$ we can take

$$
\begin{aligned}
z & =(1,0 ; \overrightarrow{0}), \\
z^{\prime} & =(0,1 ; \overrightarrow{0}), \\
w_{2}(X) & =(0,0 ; 1, \cdots, 1) .
\end{aligned}
$$

Moreover, since $w_{2}(E)=0$ we have $\left(\alpha_{I}, z\right)=\left(\beta_{I}, z\right)=0$ for all $I\left(\alpha_{I}\right.$ and $\beta_{I}$ were introduced in equation (3.33)).

The net result of these manipulations is that the integral $\mathcal{G}(\rho)$ of $(3.32)$ can be written as:

$$
\begin{aligned}
& \frac{1}{\sqrt{2 z_{+}^{2}}} \int_{\mathcal{F}} \frac{d x d y}{y^{2}} \sum_{I} \widehat{f}_{I} \sum_{c, d \in \mathbb{Z}} \exp \left[-\frac{\pi}{2 y z_{+}^{2}}|c \tau+d|^{2}-\frac{\pi}{y z_{+}^{2}}\left(\bar{\xi}_{+}^{I}, z_{+}\right)\left(\bar{\xi}_{-}^{I}, z_{-}\right)\right] \\
& \cdot \exp \left[-\frac{\pi\left(\bar{\xi}_{+}^{I}, z_{+}\right)}{z_{+}^{2}}\left(\frac{c \tau+d}{y}\right)-\frac{\pi\left(\bar{\xi}_{-}^{I}, z_{-}\right)}{z_{+}^{2}}\left(\frac{c \bar{\tau}+d}{y}\right)\right. \\
& \left.+2 \pi i \frac{\left(\alpha_{+}^{I}, z_{+}\right)}{z_{+}^{2}} c-i \pi\left(\mu, \alpha^{I}\right) c\right] \\
& \text {. } \bar{\Theta}_{\left(H^{2}+\beta^{I}\right) \cap z^{\perp} / z}\left(\tau, \mu d+\alpha^{I},-\mu c ; \widetilde{P}\left(\xi^{I}\right)\right),
\end{aligned}
$$

where the vector $\xi$ is defined in (3.25). Following Borcherds, we have introduced a vector $\mu \in K$ as follows:

$$
\mu \equiv-z^{\prime}+\frac{z_{+}}{2 z_{+}^{2}}+\frac{z_{-}}{2 z_{-}^{2}}=-z^{\prime}+\frac{z_{+}-z_{-}}{2 z_{+}^{2}} .
$$

This vector satisfies $(\mu, z)=0$ and thus descends to a vector in $K$. Note that this vector is metric dependent.

We now apply the unfolding technique to the integral (8.5). One looks at the action of $S L(2, \mathbb{Z})$ on $c$ and $d$. The degenerate orbit with $c=d=0$ gets special treatment. That contribution to the theta function is modularinvariant by itself, and gives the integral over the fundamental domain of a holomorphic form divided by a power of $y$. Such an integral can be done in a standard way by integrating by parts, picking up a contribution at infinity. Orbits with $c$ and $d$ not both zero can be transformed by $S L(2, \mathbb{Z})$ to have $d=0$, giving an integral over a strip $0 \leq x \leq 1$ in the upper half plane (rather than a modular region), together with a sum on $c$ from $-\infty$ to $+\infty$, 
omitting zero. The integral over the strip is straightforward, if tedious. We give some details of the derivation in appendix $\mathrm{C}$.

In order to write the final result, we define:

$$
S^{K} \equiv S-(S, z) z^{\prime}
$$

and

$$
\psi_{I} \equiv \frac{1}{h_{I}}\left[\left(\lambda+\beta_{I}, S^{K}\right)-\left[\left(\lambda+\beta_{I}, \mu\right)\right](S, z)\right],
$$

where [.] is the greatest integer function. The final result is then (recall the notation (3.31)):

$$
\begin{aligned}
Z_{u} & =-4 \sqrt{2} \pi i\left\{4\left[\frac{f_{\infty} h_{\infty}}{1-e^{-i(S, z) / h_{\infty}}} \widetilde{\Theta}_{K, \infty}^{(\mu)}(S)\right]_{q^{0}}\right. \\
+ & {\left.\left[\frac{f_{M} h_{M}}{1-e^{-i(S, z) / h_{M}}} \widetilde{\Theta}_{K, M}^{(\mu)}(S)\right]_{q^{0}}+\left[\frac{f_{D} h_{D}}{1-e^{-i(S, z) / h_{D}}} \widetilde{\Theta}_{K, D}^{(\mu)}(S)\right]_{q^{0}}\right\}, }
\end{aligned}
$$

where $\widetilde{\Theta}$ is a theta-function-like object

$$
\begin{array}{r}
\widetilde{\Theta}_{K, I}^{(\mu)}(S)=\sum_{\lambda \in K} \exp \left\{-i \pi \tau\left(\lambda+\beta_{I}\right)^{2}+2 \pi i\left(\lambda+\beta_{I}, \alpha_{I}\right)-i\left(\lambda+\beta_{I}, S^{K}\right) / h_{I}\right\} \\
\cdot \exp \left\{i \frac{(S, z)}{h_{I}}\left[\left(\lambda+\beta_{I}, \mu\right)\right]\right\}
\end{array}
$$

and $f_{I}$ is the holomorphic version of the function $\widehat{f}_{I}$ introduced in (3.26). (That is, we replace $\widehat{T} \rightarrow T$ in (3.26).)

There are several remarks which should be made about this result.

1. In order to apply the unfolding method we must regard the expressions as power series in $p, S$. The decay from the $\Theta$ function $\sim \exp \left[-\frac{\pi}{2 z_{+}^{2}} \frac{1}{y}\right]$ in (8.5) must not be overwhelmed by the "tachyon" divergence of the modular functions. At order $p^{\ell} S^{r}$ the modular functions diverge like:

$$
\exp \left[-\frac{2 \pi}{y}(8+\sigma)\right]
$$

in the regions $(\infty, 0),(\infty, 2)$ and like

$$
\exp \left[\frac{2 \pi}{8 y}(r+2 \ell+3)\right]
$$


in the regions $(\infty, 1),(\infty, 3), M, D$. Therefore, for $b_{-} \leq 9$ we have the condition:

$$
z_{+}^{2}<\frac{2}{r+2 \ell+3}
$$

and for $b_{-}>9$ we have an extra condition:

$$
z_{+}^{2}<\frac{1}{4\left(b_{-}-9\right)}
$$

for the validity of (8.9).

2. There are two sources of chamber dependence in the answer. First, the expression is only valid in an appropriate set of chambers defined above. Second, the factor $\left[\left(\lambda+\beta_{I}, \mu\right)\right]$ prevents $\widetilde{\Theta}_{K, I}^{(\mu)}(S)$ from being a lattice theta function. Note that the vector $\mu$ depends continuously on the metric. However, $\mu$ only enters through the greatest integer function, so the expression is metric-independent within a chamber.

3. The result (8.9) bears some similarity with Conjecture 4.12 of [10].

\subsection{Example: $X=\mathbb{P}^{1} \times \mathbb{P}^{1}$}

As a special case of the above formula we write the invariants for $X=\mathbb{P}^{1} \times \mathbb{P}^{1}$. In this case $K=0$ and the expressions simplify somewhat. Moreover, only the semiclassical cusp contributes (this is related to the fact that the SW invariants vanish, since $X$ admits a metric of positive scalar curvature).

The expression (8.9) thus simplifies to:

$$
(-8) \sqrt{2} \pi i\left[f h \operatorname{coth}\left(i \frac{(S, z)}{2 h}\right)\right]_{q^{0}} \text {. }
$$

This agrees with the expression found in [10].

At first order in $S$ we have:

$$
Z_{D}=-(S, z)\left[1+\frac{17}{2^{4}} \frac{p^{2}}{2 !}+\frac{71}{2^{5}} \frac{p^{4}}{4 !}+\frac{23505}{2^{12}} \frac{p^{6}}{6 !}+\cdots\right]
$$

Similarly, we can easily extract the first few Donaldson polynomials in $S$. To do this, recall the relation between the Donaldson polynomials and the generating function of equations (1.2) and (1.3). Using this relation, we 
have:

$$
\begin{aligned}
\mathcal{D}_{2}= & -(S, z) \\
\mathcal{D}_{10}= & 5 S^{4}(S, z)-\frac{5}{2} S^{2}(S, z)^{3}+(S, z)^{5} \\
\mathcal{D}_{18}= & 252 S^{6}(S, z)^{3}-216 S^{4}(S, z)^{5}+108 S^{2}(S, z)^{7}-40(S, z)^{9} \\
\mathcal{D}_{26}= & 102960 S^{8}(S, z)^{5}-108108 S^{6}(S, z)^{7} \\
& +63180 S^{4}(S, z)^{9}-26949 S^{2}(S, z)^{11}+9345(S, z)^{13}
\end{aligned}
$$

\section{Donaldson Iinvariants of the Projective Plane}

In this section we consider the Donaldson invariants for $\mathbb{P}^{2}$. Since the SW invariants vanish (as $\mathbb{P}^{2}$ admits a metric of positive scalar curvature), the Donaldson invariants coincide with the $u$-plane integral. $\mathbb{P}^{2}$ is therefore, in a sense, as far as possible from being of simple type.

For simplicity we focus on the case $w_{2}(E)=0$. Under this condition, the integral that must be evaluated is:

$$
\frac{(S, \omega)}{32 \sqrt{2} \pi} \int_{\Gamma^{0}(4) \backslash \mathcal{H}} \frac{d x d y}{y^{3 / 2}} \frac{\vartheta_{4}^{9}}{\left(\frac{1}{2} \vartheta_{2} \vartheta_{3}\right)^{4}} \exp \left\{2 p u-\frac{1}{24} S^{2}\left[\frac{\widehat{E}_{2}}{h(\tau)^{2}}-8 u\right]\right\} \bar{\vartheta}_{4} .
$$

If we try to do this integral using the unfolding technique on, say, the lattice theta function $\vartheta_{4} \bar{\vartheta}_{4}$, we find that we are always outside the range of validity of this method. The reason is that there is no parameter $z_{+}^{2}$ to vary; indeed, it is effectively always equal to one, hence always outside the domain of validity defined by (8.13). Therefore, we need another approach. One approach is to write the integral as a total divergence and pick up the constant term at infinity. This can be done using a nonholomorphic modular form of weight $(3 / 2,0)$ introduced by Zagier. Similar integrals have been done using this form by Borcherds [11].

\subsection{Zagier's Form and Related Forms}

Zagier's nonholomorphic modular form $[23,24]$ of weight $(3 / 2,0)$ for $\Gamma_{0}(4)$ is given by

$$
\begin{aligned}
\mathbf{G}(\tau, y) & =\sum_{n \geq 0} \mathcal{H}(n) q^{n}+\sum_{f=-\infty}^{\infty} q^{-f^{2}} \frac{1}{16 \pi y^{1 / 2}} \int_{1}^{\infty} e^{-4 \pi f^{2} u y} \frac{d u}{u^{3 / 2}} \\
& =\sum_{n \geq 0} \mathcal{H}(n) q^{n}+y^{-1 / 2} \sum_{f=-\infty}^{\infty} q^{-f^{2}} \beta\left(4 \pi f^{2} y\right)
\end{aligned}
$$


where

$$
\beta(t)=\frac{1}{16 \pi} \int_{1}^{\infty} e^{-u t} \frac{d u}{u^{3 / 2}}
$$

We define Fourier coefficients by:

$$
\mathbf{G}(\tau, y) \equiv \sum_{n \in \mathbb{Z}} c(n, y) e^{2 \pi i n x}
$$

In (9.2), the $\mathcal{H}(n)$ are Hurwitz class numbers, which are closely related to the clasis numbers of imaginary quadratic fields. The $\mathcal{H}(n)$ for small $n$ are given by

$$
\begin{aligned}
\mathcal{H}(\tau)= & \sum_{n \geq 0} \mathcal{H}(n) q^{n} \\
= & -1 / 12+q^{3} / 3+q^{4} / 2+q^{7}+q^{8}+q^{11}+(4 / 3) q^{12} \\
& +2 q^{15}+3 / 2 q^{16}+q^{19}+2 q^{20}+3 q^{23}+2 q^{24} \\
& +(4 / 3) q^{27}+2 q^{28}+3 q^{31}+3 q^{32}+2 q^{35} \\
& +(5 / 2) q^{36}+4 q^{39}+2 q^{40}+\cdots
\end{aligned}
$$

The form $\mathbf{G}$ satisfies the equation:

$$
\frac{\partial}{\partial \bar{\tau}} \mathbf{G}=\frac{1}{32 \pi i} \frac{1}{y^{3 / 2}} \overline{\vartheta_{3}(2 \tau)}
$$

From Zagier's form we construct a two-dimensional representation of the modular group:

$$
\begin{aligned}
\mathbf{G}_{\mathbf{1}} & =\sum_{n \in \mathbb{Z}} c(4 n, y / 4) e^{2 \pi i n x} \\
& =\sum_{n \geq 0} \mathcal{H}(4 n) q^{n}+2 y^{-1 / 2} \sum_{f=-\infty}^{\infty} q^{-f^{2}} \beta\left(4 \pi f^{2} y\right) \\
\mathbf{G}_{\mathbf{2}} & =\sum_{n \in \mathbb{Z}} c(4 n-1, y / 4) e^{2 \pi i(n-1 / 4) x} \\
& =\sum_{n>0} \mathcal{H}(4 n-1) q^{n-1 / 4}+2 y^{-1 / 2} \sum_{f=-\infty}^{\infty} q^{-\left(f+\frac{1}{2}\right)^{2}} \beta\left(4 \pi\left(f+\frac{1}{2}\right)^{2} y\right)
\end{aligned}
$$

These form a weight $(3 / 2,0)$ representation of the modular group with ( [38], 
Theorem 5.4, [11]): ${ }^{21}$

$$
\begin{aligned}
\left(\begin{array}{l}
\mathbf{G}_{\mathbf{1}}(\tau+1) \\
\mathbf{G}_{\mathbf{2}}(\tau+1)
\end{array}\right) & =\left(\begin{array}{c}
\mathbf{G}_{\mathbf{1}}(\tau) \\
-i \mathbf{G}_{\mathbf{2}}(\tau)
\end{array}\right) \\
\left(\begin{array}{l}
\mathbf{G}_{\mathbf{1}}(-1 / \tau) \\
\mathbf{G}_{\mathbf{2}}(-1 / \tau)
\end{array}\right) & =\frac{1+i}{2} \tau^{3 / 2}\left(\begin{array}{cc}
1 & 1 \\
1 & -1
\end{array}\right)\left(\begin{array}{l}
\mathbf{G}_{\mathbf{1}}(\tau) \\
\mathbf{G}_{\mathbf{2}}(\tau)
\end{array}\right) \\
& =-\frac{1}{\sqrt{2}}(-i \tau)^{3 / 2}\left(\begin{array}{cc}
1 & 1 \\
1 & -1
\end{array}\right)\left(\begin{array}{l}
\mathbf{G}_{\mathbf{1}}(\tau) \\
\mathbf{G}_{\mathbf{2}}(\tau)
\end{array}\right)
\end{aligned}
$$

Note that

$$
\begin{aligned}
\mathbf{G}(\tau) & =\mathbf{G}_{\mathbf{1}}(4 \tau)+\mathbf{G}_{\mathbf{2}}(4 \tau) \\
\frac{\partial}{\partial \bar{\tau}} \mathbf{G}_{1} & =\frac{1}{16 \pi i} \frac{1}{y^{3 / 2}} \overline{\vartheta_{3}(2 \tau)} \\
\frac{\partial}{\partial \bar{\tau}} \mathbf{G}_{2} & =\frac{1}{16 \pi i} \frac{1}{y^{3 / 2}} \overline{\vartheta_{2}(2 \tau)}
\end{aligned}
$$

We would like to construct a form for $\Gamma^{0}(4)$ such that we can integrate by parts in (9.1). Such a form can be constructed by noting that $\mathbf{G}((\tau+1) / 2)$ will be modular for $\Gamma(2)$. Now $\Gamma(2) \cap \Gamma^{0}(4)$ is index two in $\Gamma^{0}(4)$, a coset representative being $\tau \rightarrow \frac{\tau}{\tau+1}$. Adding the transform we obtain the desired form:

$$
Q_{(\infty, 0)} \equiv \mathbf{G}\left(\frac{\tau+1}{2}\right)+\frac{1}{2} \mathbf{G}_{\mathbf{1}}\left(\frac{\tau+1}{2}\right)
$$

Thus, $Q_{(\infty, 0)}$ is modular for $\Gamma^{0}(4)$ and obeys

$$
\frac{\partial}{\partial \bar{\tau}} Q_{(\infty, 0)}=\frac{1}{8 \sqrt{2} \pi i} \frac{1}{y^{3 / 2}} \overline{\vartheta_{4}(\tau)}
$$

The sum over cosets will now involve the functions:

$$
\begin{gathered}
Q_{(\infty, 0)}=Q_{(\infty, 2)}=\mathbf{G}_{\mathbf{1}}(2 \tau)-\mathbf{G}_{\mathbf{2}}(2 \tau)+\frac{1}{2} \mathbf{G}_{\mathbf{1}}\left(\frac{\tau+1}{2}\right) \\
Q_{(\infty, 1)}=Q_{(\infty, 3)}=\mathbf{G}_{\mathbf{1}}(2 \tau)+\mathbf{G}_{\mathbf{2}}(2 \tau)+\frac{1}{2} \mathbf{G}_{\mathbf{1}}\left(\frac{\tau}{2}\right) \\
Q_{M}=Q_{D}=\frac{1}{2}\left[\mathbf{G}_{\mathbf{2}}\left(\frac{\tau}{2}\right)+\kappa \mathbf{G}_{\mathbf{2}}\left(\frac{\tau+1}{2}\right)\right]
\end{gathered}
$$

where $\kappa=e^{2 \pi i / 8}$.

\footnotetext{
${ }^{21}$ This means the functions transform like the third power of a $\vartheta$ function. We use the principal branch of the logarithm, and define $\sqrt{z}$ to have its argument in $(-\pi / 2, \pi / 2]$ for $z$ in the complex plane cut along $(-\infty, 0]$.
} 
The integral in (9.1) now can be written:

$$
\mathcal{I}=8 \pi i \sqrt{2}(S, \omega) \int_{\bar{\Gamma} \backslash \mathcal{H}} d x d y \sum_{I} \widehat{f}_{I} \frac{\partial}{\partial \bar{\tau}} Q_{I}
$$

Like Zagier's functions the $Q_{I}$ can be written as

$$
Q_{I}(\tau, y)=\mathcal{H}_{I}(\tau)+\frac{c n s t .}{y^{1 / 2}}+\mathcal{F}_{I}(\tau, y)
$$

where $\mathcal{F}_{I}(\tau, y)$ has exponential decay for $y \rightarrow \infty$ and only negative Fourier components in $x$ (which will prevent it from contributing in the computation below) and $\mathcal{H}_{I}(\tau)$ is expressed in terms of class numbers analogous to (9.5).

We would like to integrate by parts and use the rule:

$$
\int_{\mathcal{F}} d x d y \frac{\partial}{\partial \bar{\tau}} F(x, y)=+\frac{i}{2} \lim _{\Lambda \rightarrow \infty} \int_{-\frac{1}{2}}^{+\frac{1}{2}} d x F(x, \Lambda) .
$$

However, we need to take into account the nonholomorphic dependence in $\widehat{f}$. Thus, we need to generalize Zagier's form, that is, we need (nonholomorphic) modular forms $\mathbf{G}^{(\ell)}$ for $\Gamma_{0}(4)$ of weight $\left(\frac{3}{2}+2 \ell, 0\right)$ such that

$$
\frac{\partial}{\partial \bar{\tau}} \mathbf{G}^{(\ell)}=\frac{1}{32 \pi i} \frac{1}{y^{3 / 2}} \overline{\vartheta_{3}(2 \tau)} \widehat{E}_{2}^{\ell}
$$

This may be done with the aid of the following.

Lemma. Suppose $g(\tau, \bar{\tau})$ is modular of weight $(3 / 2,0)$ for some congruence subgroup $\Gamma^{\prime}$. Suppose moreover that

$$
\frac{\partial}{\partial \bar{\tau}} g(\tau, \bar{\tau})=\frac{h(\bar{\tau})}{y^{3 / 2}}
$$

Then

$$
\mathcal{E}^{\ell}[g]=\sum_{j=0}^{\ell}\left(\begin{array}{l}
\ell \\
j
\end{array}\right) \frac{\Gamma(3 / 2)}{\Gamma(3 / 2+j)}\left(\frac{6 i}{\pi}\right)^{j} E_{2}^{\ell-j}\left(\frac{d}{d \tau}\right)^{j} g
$$

is modular for $\Gamma^{\prime}$ of weight $\left(2 \ell+\frac{3}{2}, 0\right)$ and satisfies:

$$
\frac{\partial}{\partial \bar{\tau}} \mathcal{E}^{\ell}[g]=\frac{h(\bar{\tau})}{y^{3 / 2}} \widehat{E}_{2}^{\ell} .
$$

Proof: It is straightforward to show (9.19) by differentiating and using the fact that $h(\bar{\tau})$ does not depend on $\tau$. It is not obvious that the expression in (9.1.8) transforms well under modular transformations. However, (9.18) 
may be expressed in terms of Cohen's operator [39], which is essentially just a product of covariant derivatives normal-ordered. Cohen's operator is:

$$
\mathcal{F}_{r}[f]=\sum_{j=0}^{r}\left(\begin{array}{l}
r \\
j
\end{array}\right) \frac{\Gamma(k+r)}{\Gamma(k+j)}\left(\frac{1}{2 i y}\right)^{r-j}\left(\frac{d}{d \tau}\right)^{j} f,
$$

If $f$ is modular of weight $(k, 0)$ then $\mathcal{F}_{r}[f]$ is modular of weight $(k+2 r, 0)$. It is straightforward to check that

$$
\mathcal{E}^{\ell}[g]=\sum_{t=0}^{\ell}\left(\begin{array}{l}
\ell \\
t
\end{array}\right) \frac{\Gamma(3 / 2)}{\Gamma(3 / 2+\ell-t)}\left(\frac{6 i}{\pi}\right)^{\ell-t} \widehat{E}_{2}^{t} \mathcal{F}_{\ell-t}[g]
$$

and thus $\mathcal{E}^{\ell}[g]$ is modular.

\subsection{Answer for $\mathbb{P}^{2}$}

It is now straightforward to evaluate the integral for $\mathbb{P}^{2}$. One expands the exponential $\exp \left[-S^{2} \widehat{E}_{2} /\left(24 h^{2}\right)\right]$ in powers of $S^{2}$ and expresses each term as a total derivative using the lemma. Then integrating by parts, and keeping the zeroth Fourier coefficient, one obtains a double sum. This is easily rewritten so that one can re-exponentiate one infinite sum to get a factor $\exp \left[-S^{2} E_{2} /\left(24 h^{2}\right)\right]$. The result of all these manipulations is the formula:

$$
Z_{D}=-4 \sqrt{2} \pi(S, \omega) \sum_{j=0}^{\infty} \frac{\Gamma(3 / 2)}{j ! \Gamma(3 / 2+j)}\left(\frac{S^{2}}{2}\right)^{j}\left[\sum_{I} \frac{f_{I}}{h_{I}^{2 j}}\left(q \frac{d}{d q}\right)^{j} \mathcal{H}_{I}(\tau)\right]_{q^{0}} .
$$

Examining the sum over cosets we find the entire contribution comes from the semiclassical cusp. Consequently, the $S U(2)$ Donaldson polynomials for $\mathbb{P}^{2}$ are given by:

$$
\begin{aligned}
\mathcal{D}_{w_{2}(E)=0}(S, p)= & \sum_{x, t} S^{2 x+1} p^{t} d_{2 x+1, t} \\
d_{2 x+1, t}= & -2^{t-1}(2 x+1) ! \sum_{j=0}^{x} \frac{1}{(x-j) !} \cdot \frac{\Gamma(3 / 2)}{j ! \Gamma(3 / 2+j)}\left(\frac{1}{2}\right)^{j} \\
& \cdot\left[\frac{\vartheta_{4}^{9}}{h^{4+2 j}} T^{x-j} u^{t} \mathcal{H}_{(\infty, 0)}(q, j)\right]_{q^{0}}, \\
\mathcal{H}_{(\infty, 0)}(q, j) \equiv & \left(q \frac{d}{d q}\right)^{j} \mathcal{H}_{(\infty, 0)}(q), \\
\mathcal{H}_{(\infty, 0)}= & \sum_{n \geq 0} H(4 n) q^{2 n}+\frac{1}{2} \sum_{n \geq 0} H(4 n) q^{n / 2}(-1)^{n} \\
& -\sum_{n>0} H(4 n-1) q^{2 n-1 / 2} .
\end{aligned}
$$


Expanding, we find that the first four polynomials are:

$$
\begin{aligned}
\mathcal{D}_{2}= & -\frac{3}{2} S \\
\mathcal{D}_{10}= & S^{5}-p S^{3}-\frac{13}{8} p^{2} S \\
\mathcal{D}_{18}= & 3 S^{9}+\frac{15}{4} S^{7} p-\frac{11}{16} S^{5} p^{2}-\frac{141}{64} S^{3} p^{3}-\frac{879}{256} S p^{4} \\
\mathcal{D}_{26}= & 54 S^{13}+24 S^{11} p+\frac{159}{8} S^{9} p^{2} \\
& +\frac{51}{16} S^{7} p^{3}-\frac{459}{128} S^{5} p^{4}-\frac{1515}{256} S^{3} p^{5}-\frac{36675}{4096} S p^{6}
\end{aligned}
$$

in agreement with the results obtained previously in $[25,15]$.

\subsection{Class number relations}

In [15], Göttsche gave a closed expression for the Donaldson invariants for $\mathbb{P}^{2}$. His expression differs from the above, and comparing the two implies relations on class numbers similar to the famous relations of Kronecker, Weber, and Zagier. See, for instance [23, 24].

The answer obtained above in terms of class numbers is:

$$
\operatorname{Res}_{q=0}\left(\frac{d q}{q} \cdot\left(-\frac{1}{2}\right) \sum_{j=0}^{\infty} \frac{\Gamma(3 / 2)}{j ! \Gamma(3 / 2+j)} \frac{S^{2 j+1}}{2^{j}}\left[e^{2 p u+S^{2} T} \frac{\vartheta_{4}^{9}}{h^{4+2 j}} \mathcal{H}_{(\infty, 0)}(q, j)\right]\right) \text {. }
$$

In [15], Göttsche first blows up $\mathbb{P}^{2}$ to $\mathbb{F}_{1}$ and (in the notation of section 5) considers an $S O(3)$ bundle $\widetilde{E}$ with $w_{2}(\widetilde{E})=F$ in the chamber $\omega \cdot B=0$, indeed, $\omega=H$. This is on the wrong side of the Kähler cone to apply the vanishing theorem. He then adds all the wall crossings in the Kähler cone to go to $\omega=F$ where the vanishing theorem applies. The walls are $W_{\lambda}$ for $\pm \lambda=\left(n_{2}+\frac{1}{2}\right) B-n_{1} H$ with $n_{1}>0, n_{2} \geq n_{1}$. The answer obtained for the generating function of $\mathbb{P}^{2}$ Donaldson invariants is

$$
\operatorname{Res}_{q=0}\left(\frac{d q}{q} \cdot\left(-\frac{1}{2}\right) \sum_{j=0}^{\infty} \frac{(-1)^{j} S^{2 j+1}}{(2 j+1) !}\left[e^{2 p u+S^{2} T} \frac{\vartheta_{4}^{8}}{h^{4}} V(q, j)\right]\right)
$$

where

$$
V(q, j)=\sum_{n_{1}>0, n_{2} \geq n_{1}}(-1)^{n_{1}+n_{2}}\left(2 n_{2}+1\right) n_{1}^{2 j+1} \frac{q^{\frac{1}{2}\left(n_{2}\left(n_{2}+1\right)-n_{1}^{2}\right)+1 / 8}}{h^{2 j+1}} .
$$


It is tempting to try to cancel the common factors $e^{2 p u+S^{2} T} \frac{\vartheta_{4}^{8}}{h^{4}}$ in $(9.25)$ and (9.26) and then equate the power series in $S, q$. This gives false formulae because the function $e^{2 p u+S^{2} T} \frac{\vartheta_{4}^{8}}{h^{4}}$ is not sufficiently generic. A safe way to proceed is to note that the function $e^{2 p u}$, when expanded in powers of $p$, generates arbitrary non-negative powers of $u$. Hence, it is helpful to change variables from $q$ to $u$, and replace the extraction of residues at $q=0$ with residues at $u=\infty$, using the relation

$$
\frac{d q}{q}=\frac{d u}{u} \cdot \frac{u}{q} \frac{d q}{d u} .
$$

Then, equality of residues at $u=\infty$ for all $p$ means that the functions multiplying $e^{2 p u}$ in the two expressions have the same non-positive terms in their Laurent expansions near $u=\infty$. Thus:

$$
\begin{aligned}
{\left[\left(\frac{d q}{d u} \frac{u}{q}\right) e^{S^{2} T} \frac{\vartheta_{4}^{8}}{h^{4}} \sum_{j=0}^{\infty}\right.} & \left.\frac{\Gamma(3 / 2)}{j ! \Gamma(3 / 2+j)} \frac{S^{2 j+1}}{2^{j} h^{2 j}} \vartheta_{4} \mathcal{H}_{(\infty, 0)}(q, j)\right]_{\leq 0}= \\
& {\left[\left(\frac{d q}{d u} \frac{u}{q}\right) e^{S^{2} T} \frac{\vartheta_{4}^{8}}{h^{4}} \sum_{j=0}^{\infty} \frac{(-1)^{j} S^{2 j+1}}{(2 j+1) !} V(q, j)\right]_{\leq 0} . }
\end{aligned}
$$

We need care here, since $T(u)$ and other functions appearing here have series expansions in inverse powers of $u$, but they are multiplying expressions with series in positive powers of $u$, so we cannot cancel these factors.

Nevertheless, (9.29) does imply some very interesting results on class numbers. As an example of these relations we take the term at first order in $S$. Equation (9.29) means that the functions on the left and right differ by an entire function of $u$. Taking only the linear term in $S$, we get on the left a function that is constant at $u=\infty$ and on the right a function that vanishes there, so the functions on the left and right actually differ by a constant. Using this information together with the relation

$$
\frac{1}{u} q \frac{d u}{d q}=-\frac{1}{8 u} \frac{\vartheta_{4}^{8}}{\left(\vartheta_{2} \vartheta_{3}\right)^{2}}
$$

we obtain an explicit formula for class numbers:

$$
\mathcal{H}_{(\infty, 0)}=\sum_{n_{1}>0, n_{2} \geq n_{1}}(-1)^{n_{1}+n_{2}}\left(2 n_{2}+1\right) n_{1} \frac{q^{\frac{1}{2}\left(n_{2}\left(n_{2}+1\right)-n_{1}^{2}\right)+1 / 8}}{\eta^{3}}-\frac{\vartheta_{2}^{4}+\vartheta_{3}^{4}}{8 \vartheta_{4}} .
$$

We have checked this numerically to order $q^{11 / 2}$. It would be very interesting to see if this relation leads to improved estimates on the asymptotic behavior of class numbers. 


\section{Extension to $\pi_{1}(X) \neq 0$}

We indicate here how some of the above results generalize to Donaldson theory on a four-manifold $X$ that is not simply-connected and which in general has $b_{1} \neq 0$. For simplicity, we assume that there is no two-torsion in $H^{2}(X, \mathbb{Z})$, so that $w_{2}(E)$ has an integral lift and some of our formulas which involve dividing by two still make sense.

When $b_{1}(X) \neq 0$, we can add new operators to the microscopic correlation function, namely $\int_{\gamma} \operatorname{Tr} \phi \psi=\int_{\gamma} K u$ for a one-cycle $\gamma$ and $\int_{L} K^{3} u$ for a three-cycle $L$. To compute correlation functions of such operators, one will have to determine several new contact term corrections. For simplicity we omit these operators.

On general grounds the photon partition function always involves a sum over all line bundles. Line bundles are classified topologically by $c_{1} \in$ $H^{2}(X ; \mathbb{Z})$. Moreover, on each class we must integrate over the moduli space of harmonic connections. This moduli space is a torus of dimension $b_{1}(X)$.

Proceeding as in section three, we reduce the evaluation of the $u$-plane contribution to the path integral to the finite-dimensional integral:

$$
\begin{gathered}
Z_{u}=2 \int[d a d \bar{a} d \eta d \chi] \int_{P i c(X)} d \psi \int d D A^{\chi} B^{\sigma} y^{-1 / 2} \\
\cdot \exp \left[\frac{1}{8 \pi} \int(\operatorname{Im} \tau) D \wedge * D\right] \exp \left[-i \pi \bar{\tau} \lambda_{+}^{2}-i \pi \tau \lambda_{-}^{2}+\pi i\left(\lambda, w_{2}(X)\right)\right] \\
\cdot \exp \left[-\frac{i \sqrt{2}}{16 \pi} \int \frac{d \bar{\tau}}{d \bar{a}} \eta \chi \wedge\left(D_{+}+4 \pi \lambda_{+}\right)+\frac{i \sqrt{2}}{2^{7} \pi} \int \frac{d \tau}{d a}(\psi \wedge \psi) \wedge\left(4 \pi \lambda_{-}+D_{+}\right)\right. \\
\left.+\frac{1}{3 \cdot 2^{11} \pi i} \int \frac{d^{2} \tau}{d a^{2}} \psi \wedge \psi \wedge \psi \wedge \psi+2 p u+\frac{i}{\sqrt{2} \pi} \int_{S} K^{2} u+S^{2} T(u)\right] \cdot
\end{gathered}
$$

Here $\int_{P i c(X)}$ is the sum/integral over all pairs consisting of a complex line bundle on $X$ together with a harmonic connection. These bundles have first Chern class $2 \lambda \in H^{2}(X ; \mathbb{Z})$ which is congruent to $w_{2}(E)$ modulo two. In other words, $\int_{P i c(X)}$ is a sum over $H^{2}(X, \mathbb{Z})$ times an integral over a $b_{1}(X)$-dimensional torus. The $\psi$ zero modes are tangent to $\operatorname{Pic}(X)$, so the integration over them is naturally understood as the integral of a differential form on Pic $(X)$. As for the $\eta$ and $\chi$ zero modes, they are normalized as in section 2.3 to represent fixed cohomology classes (which means in practice that the zero mode wave function for $\eta$ is $\eta_{0}=1$, and for $\chi$ we use a basis of orthonormal self-dual harmonic two-forms). The integral over $D$ is over the space of self-dual two-forms. The "integral" over $D$ is really a Gaussian integral with an exponent of the wrong sign and is to be interpreted 
algebraically. (Also, the $D$ determinant should be ignored; this is part of the supersymmetric cancellation of all the bose and fermi determinants.)

The integrals in (10.1) are most readily done by first doing the formal Gaussian integral on the auxiliary field $D$ and then soaking up the fermion zero modes. The resulting expressions may be seen to be modular invariant if we treat $\psi$ as weight $(1,0)$. Indeed, making the simple redefinition

$$
\widetilde{S} \equiv S-\frac{\sqrt{2}}{32} \frac{d \tau}{d u} \psi \wedge \psi
$$

we get an expression with the same $F, D, \chi, \eta$ dependence as in the simply connected case. Therefore we can say without further ado that the $u$-plane integral is:

$$
Z_{u}=\int_{\Gamma^{0}(4) \backslash \mathcal{H}} \frac{d x d y}{y^{1 / 2}} \mu(\tau) \int_{P i c(X)} d \psi e^{2 p u+\widetilde{S}^{2} \widehat{T}(u)+\left(\widetilde{S}, \psi^{2}\right) H(u)+\psi^{4} K(u)} \Psi(\widetilde{S})
$$

where

$$
\begin{aligned}
\mu(\tau)=- & \frac{\sqrt{2}}{2} \frac{d a}{d \tau} A^{\chi} B^{\sigma} \\
\Psi(\widetilde{S})= & \exp \left(2 i \pi \lambda_{0}^{2}\right) \exp \left[-\frac{1}{8 \pi y}\left(\frac{d u}{d a}\right)^{2} \widetilde{S}_{-}^{2}\right] \\
& \cdot \sum_{\lambda \in H^{2}+\frac{1}{2} w_{2}(E)} \exp \left[-i \pi \bar{\tau}\left(\lambda_{+}\right)^{2}-i \pi \tau\left(\lambda_{-}\right)^{2}+\pi i\left(\lambda-\lambda_{0}, w_{2}(X)\right)\right] \\
& \cdot \exp \left[-i \frac{d u}{d a}\left(\widetilde{S}_{-}, \lambda_{-}\right)\right]\left[\left(\lambda_{+}, \omega\right)+\frac{i}{4 \pi y} \frac{d u}{d a}\left(\widetilde{S}_{+}, \omega\right)\right] \\
H(u)= & \frac{i \sqrt{2}}{64 \pi}\left(\frac{d^{2} u}{d a^{2}}-4 \pi i \frac{d \tau}{d u} T(u)\right) \\
K(u)=- & \frac{i}{3 \cdot 2^{11} \pi}\left(\frac{d^{2} \tau}{d a^{2}}-6 \frac{d \tau}{d u} \frac{d^{2} u}{d a^{2}}+12 \pi i\left(\frac{d \tau}{d u}\right)^{2} T(u)\right)
\end{aligned}
$$

It is easy to check that, although $T(u)$ transforms by a shift under modular transformations, $H(u)$ and $K(u)$ transform covariantly with weights $(-2,0),(-4,0)$ respectively. Indeed, using

$$
a=\frac{1}{6}\left(\frac{2 E_{2}+\vartheta_{2}^{4}+\vartheta_{3}^{4}}{\vartheta_{2} \vartheta_{3}}\right)
$$


one can derive the explicit $q$-expansions:

$$
\begin{aligned}
& H(u)=\frac{i \sqrt{2}}{16 \pi} \frac{\vartheta_{2}^{4}+\vartheta_{3}^{4}}{\vartheta_{4}^{8}} \\
& K(u)=-\frac{7}{3 \cdot 2^{7} \pi^{2}} \frac{\left(\vartheta_{2} \vartheta_{3}\right)^{2}\left(\vartheta_{2}^{4}+\vartheta_{3}^{4}\right)}{\vartheta_{4}^{16}} .
\end{aligned}
$$

This allows us to extend immediately all the above results on wall-crossing, blow-up, explicit evaluations, and the like, to the case $b_{1}(X)>0$, since the integral has the same form. In fact, the above formulae can be further simplified, since for any four elements $\psi_{1}, \ldots, \psi_{4}$ of $H^{1}(X ; \mathbb{R})$ we have $\psi_{1} \wedge$ $\cdots \wedge \psi_{4}=0$ when $b_{2}^{+}<3$. Hence we may drop the $\psi^{4}$ terms. (We have given the formulae for $K$ since they are likely to be useful in related contexts.)

The discussion of section 7 can also be extended to the non-simplyconnected case. Define

$$
\widetilde{S}_{M} \equiv S-\frac{\sqrt{2}}{32} \frac{d \tau_{D}}{d u} \psi \wedge \psi .
$$

Then, using (7.16) one finds the generalization of (7.11) to be

$$
\begin{aligned}
\left\langle e^{p \mathcal{O}+I(S)}\right\rangle_{\xi, u=1}= & \int_{P i c(X)} d \psi \int_{\mathcal{M}_{\lambda}} 2 e^{2 i \pi\left(\lambda_{0} \cdot \lambda+\lambda_{0}^{2}\right)} C(u)^{\lambda^{2} / 2} P(u)^{\sigma / 8} L(u)^{\chi / 4} \\
& \cdot \exp \left(H_{M}\left(\widetilde{S}_{M}, \psi^{2}\right)+\frac{\sqrt{2}}{2^{6} \pi^{2}} \frac{1}{a_{D}}\left(\psi^{2}, \lambda\right)\right) \\
& \cdot \exp \left(2 p u+i \frac{d u}{d a_{D}}\left(\widetilde{S}_{M}, \lambda\right)+\widetilde{S}_{M}^{2} T_{M}(u)\right),
\end{aligned}
$$

where the line bundles in $\operatorname{Pic}(X)$ now have $2 \lambda \in H^{2}(X ; \mathbb{Z})$ congruent to $w_{2}(X)$ modulo two, and we have dropped two $\psi^{4}$ terms since $b_{2}^{+}=1$.

We can now turn the reasoning of section seven around and use (10.3) and (10.8) to give a new derivation of the generalized wall-crossing formulae for Seiberg-Witten invariants given in [40, 41].

\section{Incorporation of Matter}

$\mathcal{N}=2$ supersymmetric gauge theories in four dimensions can be generalized to include hypermultiplets in some representation of the gauge group. Insuring that the beta function should be zero or negative places a restriction on the possible representations. For the case that the gauge group is $S U(2)$, the 
possibilities are that the hypermultiplets consist of $2 N_{f}$ copies of the twodimensional representation of $S U(2)$, for $N_{f} \leq 4,{ }^{22}$ or a single copy of the adjoint representation. These theories allow hypermultiplet bare masses and all have the $S U(2)_{R}$ group of $R$ symmetries. An additional $U(1)_{R}$ symmetry group is generally anomalous and is also explicitly violated by hypermultiplet bare masses. The theory with the adjoint hypermultiplet actually has more symmetry $\left(\mathcal{N}=4\right.$ supersymmetry and $S O(6)_{R}$ in the absence of a hypermultiplet bare mass, broken to $\mathcal{N}=2$ and $S O(4)_{R}$ if there is a bare mass); we will call it the $\mathcal{N}=4$ theory (though we are mainly interested in the case in which the $\mathcal{N}=4$ is softly broken to $\mathcal{N}=2$ by the bare mass).

After including hypermultiplets, the Coulomb branch of vacua is still parametrized by a copy of the $u$-plane (where $u$ is related to $\left\langle\operatorname{Tr} \phi^{2}\right\rangle$ in the underlying theory), but now the $u$-plane parametrizes a different family of elliptic curves. The appropriate families (which depend on the hypermultiplet bare masses) were determined in [3]. They have the form:

$$
y^{2}=x^{3}+a_{2} x^{2}+a_{4} x+a_{6},
$$

where $a_{2}, a_{4}, a_{6}$ are polynomials in $u$ and in the masses $m_{i}$. They are also polynomials in the scale $\Lambda$ of the theory for $N_{f}<4$, or of certain modular functions $e_{i}\left(\tau_{0}\right)$ for $N_{f}=4$ or for $\mathcal{N}=4$. Here $\tau_{0}$ will refer to the coupling as measured at $u=\infty$ in the $N_{f}=4$ or $\mathcal{N}=4$ theory. In this paper we have put $\Lambda=1$ for $N_{f}=0$.

Any of these theories can be twisted to obtain a topological field theory. We will consider here only the standard twist, which as reviewed in section 2 is obtained by decomposing the four-dimensional rotation group as $\operatorname{Spin}(4)=S U(2)_{-} \times S U(2)_{+}$and then picking a diagonal subgroup of $S U(2)_{+} \times S U(2)_{R}$. Some additional twists are possible, using the $\operatorname{Spin}\left(2 N_{f}\right)$ global symmetry in the case of matter multiplets in the two-dimensional representation and picking a homomorphism of $S U(2)_{+}$to $\operatorname{Spin}\left(2 N_{f}\right)$, or using the extended symmetry of the $\mathcal{N}=4$ theory. (An alternative twist in the latter case, related to the Euler characteristic of instanton moduli space, was explored in [42].)

The invariants of smooth four-manifolds associated with these twisted theories with hypermultiplets could be computed at short distances in terms of the underlying $S U(2)$ gauge theory with matter. Such an analysis would proceed roughly along the lines in $[42,28]$ and will not be explored here. Our goal will be to compute at long distances in terms of the physical vacua. We

\footnotetext{
${ }^{22}$ The number of copies of the two-dimensional representation must be even; otherwise the quantum theory is inconsistent because of a global anomaly. A single copy of the two-dimensional representation gives what is sometimes called a half-hypermultiplet. A pair of half-hypermultiplets is sometimes called a quark or a quark flavor, a terminology we will sometimes use below.
} 
will consider mainly the case that the hypermultiplet bare masses are generic, so that there is no Higgs branch of vacua, and the entire contribution comes from the Coulomb branch. On the Coulomb branch, there is a finite set of exceptional points at which a massless charged hypermultiplet appears. There will be SW contributions from these points, which can be analyzed rather as in section 7 . There is also a continuous $u$-plane integral, similar to the ones we have already studied but with some differences that we will analyze.

We will obtain the analogue of (3.20) for the theories with hypermultiplets. It is given by equation (11.8) below. Then we will discuss special properties of the integrals and of the various models.

\subsection{The Measure Factor and the Contact Term}

First we analyze the measure factor $A^{\chi} B^{\sigma}$ for the $u$-plane integral with hypermultiplets.

The factor $B^{\sigma}$, for the theory without hypermultiplets, was determined in [33] as follows. We will express the argument in a way that carries over immediately to the general case. This factor has neither zeroes nor poles on the $u$-plane except at points $u_{i}$ at which there is a massless charged hypermultiplet. Integrating out the light hypermultiplet produces a singularity $B^{\sigma} \sim\left(u-u_{i}\right)^{\sigma / 8}$. Hence, if we $\operatorname{set}^{23}$

$$
\Delta=\prod_{i}\left(u-u_{i}\right)
$$

then up to a constant multiplicative factor one has

$$
B^{\sigma}=\Delta^{\sigma / 8} .
$$

The case treated in [33] was the case $\Delta=u^{2}-1$. There is no way to determine an overall multiplicative factor in (11.3) except by comparing to a precise definition of the theory at short distances; we have done so earlier in this paper for the pure gauge theory, but will not do so for the theories with hypermultiplets.

Just as in [33], this formula can be checked by looking at the behavior near $u=\infty$. Near $\infty$, the function $B^{\sigma}$ should behave as a power of $u$, in a way that reproduces the anomaly of those of the elementary fields

\footnotetext{
${ }^{23}$ We recall from [3] that for the theory with doublet hypermultiplets, one uses a formalism that generalizes the $\Gamma^{0}(4)$ formalism of the pure gauge theory. In this description, the discriminant of the family of elliptic curves is up to a constant multiple the function $\Delta$ defined in the next equation. For the $\mathcal{N}=4$ theory, it is more convenient to use instead a formalism related to $\Gamma(2)$, and then the discriminant is the square of what we are here calling $\Delta$.
} 
that are massive at large $u$ and have been integrated out to produce the $B$ function. (Possible hypermultiplet bare masses which break the $U(1)_{R}$ symmetry explicitly are irrelevant at large $u$.) The charged components of the vector multiplet give an anomaly that corresponds to a behavior at infinity $B^{\sigma} \sim u^{\sigma / 4}$, as in eqn. (3.5) of [33]. Including the contributions of charged components of the hypermultiplets, the behavior at infinity should be $B^{\sigma} \sim u^{\left(2+N_{f}\right) \sigma / 8}$ for the theory with $2 N_{f}$ doublets, or $u^{3 \sigma / 8}$ for the theory with an adjoint hypermultiplet. This agrees with (11.3), since [3] $\Delta$ is a polynomial of degree $2+N_{f}$ or of degree 3 in the two cases.

Now we consider the "function" $A^{\chi}$. This quantity was determined in [33] from the following properties:

(1) It is actually not a function in the ordinary sense, but transforms under duality transformations in the low energy theory like a holomorphic modular form of weight $-\chi / 2$.

(2) In the appropriate local description of the low energy theory, it has neither zeroes nor poles at zeroes of $\Delta$ or elsewhere on the $u$-plane.

(3) It behaves near $u=\infty$ as $u^{\chi / 4}$.

All of these properties are unchanged by the incorporation of hypermultiplets. In fact, the anomalies of the elementary hypermultiplets involve only $\sigma$ and not $\chi$, so incorporation of such hypermultiplets does not modify assertion (3). Likewise, massless charged hypermultiplets of the low energy theory have an anomaly that is independent of $\chi$, which is the reason for the assertion in (2) that $A^{\chi}$ is regular and non-zero even at zeroes of $\Delta$. Finally, because the hypermultiplet kinetic energy has no explicit $\tau$-dependence, the analysis of the modular weights proceeds just as in [33], leading to assertion (1).

One would therefore expect that in some sense $A^{\chi}$ would be the same as in the theory without hypermultiplets. That is so, but some care is required. The result in [33] was $A^{\chi}=\left(\left(u^{2}-1\right) d \tau / d u\right)^{\chi / 4}$.

The most obvious thing to do is to replace $u^{2}-1$ by $\Delta$. However, it is not true that $A^{\chi}$ is equal to $(\Delta d \tau / d u)^{\chi / 4}$. This fails to obey property (2), which fails at zeroes of $d \tau / d u$. (For the theory without elementary hypermultiplets, there are no such zeroes, as shown on p. 398 of [33] by an argument that does not carry over when hypermultiplets are included.) But we can proceed as follows. For the theory without hypermultiplets, there is an identity [43]:

$$
\left(u^{2}-1\right) \frac{d \tau}{d u}=\frac{i}{4 \pi}\left(\frac{d u}{d a}\right)^{2} .
$$

Hence the result in [33] could have been written

$$
A^{\chi}=\left(\frac{d u}{d a}\right)^{\frac{\chi}{2}} \text {. }
$$


This expression obeys properties (1)-(3), irrespective of the presence of matter hypermultiplets. Property (1) is verified using the fact that $d u / d a_{D}=$ $\left(d a / d a_{D}\right) d u / d a=(1 / \tau) d u / d a$. As regards $(2)$, the absence of zeroes or poles of $A^{\chi}$ away from zeroes of $\Delta$ follows from the fact that $d a / d u$ is a period of a holomorphic differential and so is never zero. Regularity at zeroes of $\Delta$ follows from the fact that, in the appropriate local description, $a$ is a good local coordinate at such a zero so $d u / d a \neq 0$. Finally, (3) is a consequence of the fact that near infinity $a \sim \sqrt{u}$.

To summarize our results so far, the measure factor is

$$
A^{\chi} B^{\sigma}=\left(\frac{d u}{d a}\right)^{\chi / 2} \Delta^{\sigma / 8}
$$

up to multiplicative constants that depend on a precise microscopic definition of the theory.

The other somewhat similar function that must be determined is the contact function $T(u)$ that arises in the product of two-observables. The derivation in section 2.2 , which led in equation (2.25) to the general structure

$$
T=-\frac{1}{24} E_{2}(\tau)\left(\frac{d u}{d a}\right)^{2}+H(u)
$$

carries over here. We recall that $H$ is here an ordinary holomorphic function of $u$. Moreover the determination of the function $H$ that was given in section 2.2 for the theory without hypermultiplets carries over with only small modifications to the general case. One modification is that in general the theory with hypermultiplets has no symmetry under $u \rightarrow-u$. An examination of the determination of $H$ for $N_{f}=0$ shows that the same result that is, $H(u)=u / 3$ - follows without any assumption of this symmetry if $T$ vanishes for $u \rightarrow \infty$. This is so by asymptotic freedom for doublet hypermultiplets with $N_{f}=1,2,3$, so the form of $T(u)$ obtained previously for $N_{f}=0$ carries over to these cases. For $N_{f}=4$, or for $\mathcal{N}=4$, instead of asymptotic freedom one has conformal invariance near $u=\infty$. In those cases, instead of vanishing near infinity, $T$ might approach a constant (independent of $u$, but depending on the bare coupling constant or more precisely on the coupling constant $\tau_{0}$ measured at $u=\infty$ ). Thus for $N_{f}=4$ or $\mathcal{N}=4$, we have $H(u)=E_{2}\left(\tau_{0}\right) \cdot u / 3$ up to a possible additive constant. The coefficient of $u$ has been chosen to cancel a pole in $T$ at $u=\infty$. Since topological invariance would not be spoiled by adding a constant to $T$, the constant term in $T(u)$ can only be determined in these examples by comparing to a microscopic definition of the theories, or possibly by using $S$-duality and holomorphy in $\tau$. 


\subsection{Expression for the $u$-Plane Integrals}

We now consider the twisted theories with hypermultiplets on a four-manifold $X$ with $b_{1}=0, b_{2}^{+}=1$. For the theories with doublet hypermultiplets, one must set $\xi=w_{2}(E)=w_{2}(X)$. The reason is that the hypermultiplets, being doublets, transform non-trivially under the center of the gauge group $S U(2)$ and, being spinors, also transform under the center of the cover of the Lorentz group. (For some alternative twists that use suitable homomorphisms of $S U(2)_{+}$to $\operatorname{Spin}\left(2 N_{f}\right)$, for $N_{f}=2,4$, this restriction would be modified.) For the case of the adjoint hypermultiplet, the hypermultiplets transform trivially under the center of $S U(2)$ but are still spinors with respect to the Lorentz group. Thus these theories only make sense for $w_{2}(X)=0$, i.e., for spin manifolds.

Apart from factors examined in the last subsection, the derivation of the $u$-plane integrand in these theories proceeds rather as in section 3. In particular, the definition of the photon path integral $Z$ of eqn. (3.19) is unchanged. One important difference, which leads to some complications in actually performing integrals, is that the $u$-plane is generically not a modular curve, and hence one cannot conveniently map the integration region to a fundamental domain in the upper half $\tau$ plane, as we did in the theory without elementary hypermultiplets.

Putting together all the above remarks, we conclude that the $u$-plane integral for all values of $N_{f}$ is given by:

$$
Z_{u}\left(p, S ; m_{i}, \tau_{0}\right)=\int_{\mathbb{C}} \frac{d u d \bar{u}}{y^{1 / 2}} \mu(\tau) e^{2 p u+S^{2} \widehat{T}(u)} \Psi .
$$

(For $N_{f}<4$ we replace $\tau_{0} \rightarrow \Lambda$ on the left hand side.) The function $\Psi$ is exactly the same as (3.18). The measure is now:

$$
\mu(\tau)=2 \alpha^{\chi} \beta^{\sigma} \frac{d \bar{\tau}}{d \bar{u}}\left(\frac{d a}{d u}\right)^{1-\frac{1}{2} \chi} \Delta^{\sigma / 8} .
$$

Here $\alpha, \beta$ are functions of $m_{i}, \Lambda$ for $N_{f}<4$ and functions of $m_{i}, \tau_{0}$ for $N_{f}=$ 4. It is possible that they can be determined by constraints of symmetry, holomorphy, and RG flow. (We hope to return to this in future work.) Of course, the definition of $Z_{u}$ also depends implicitly on a choice of metric through a choice of period point $\omega$. We will study the dependence on $\omega$ presently.

As for $N_{f}=0$, the integral (11.8) requires precise definition. There are singularities in the integral near the zeroes of $\Delta$ and near $u=\infty$. Near the zeroes of $\Delta$ one can express the integrand in terms of the appropriate $\tau$ parameter and use the definition discussed in section 3.2. This also works at $u=\infty$ for $N_{f}<4$. 
For $N_{f}=4$ (or similarly $\mathcal{N}=4$ ) the $\tau$ parameter behaves at $u \rightarrow \infty$ like:

$$
\tau\left(u ; m_{i}, \tau_{0}\right)=\tau_{0}+\mathcal{O}(1 / u)
$$

We also have:

$$
\frac{d a}{d u} \rightarrow \frac{1}{\sqrt{8 u}}\left(1+\mathcal{O}\left(\frac{1}{u}\right)\right)
$$

Finally, $\Delta$ is a sixth order polynomial in $u$. Hence the measure behaves as

$$
F\left(m_{i}, \tau_{0}\right) d u d \bar{u} \frac{1}{\bar{u}^{2}} u^{-\frac{1}{2}+\chi / 4+3 \sigma / 4}\left(1+\mathcal{O}\left(\frac{1}{u}, \frac{1}{\bar{u}}\right)\right),
$$

for some function $F$. The series in $\frac{1}{\bar{u}}$ comes from the expansion of $y=\operatorname{Im} \tau$ and $d \bar{\tau} / d \bar{u}$. An operator insertion of ghost number $Q$ modifies the integrand at $u \rightarrow \infty$ by an insertion of $u^{Q / 4}$. This is always holomorphic. Therefore, in order to define the integral at infinity we first integrate over the region $|u|<R$ and then take $R \rightarrow \infty .{ }^{24}$ The integrand of (11.8) has an expansion in terms $\sum_{\mu, \nu} u^{\mu} \bar{u}^{\nu}$, where the largest possible value of $\nu$ is -2 . Hence, upon integrating over a large circle in the $u$-plane with $|u|$ fixed and then integrating over $|u|$, the dangerous terms vanish and one gets a convergent result.

\subsection{Topological Invariance of the Integrals}

We will now investigate the topological invariance of the integral (11.8). Actually, anticipating that wall-crossing at zeroes of $\Delta$ will cancel similar behavior of SW contributions, we will focus on the behavior near $u=\infty$. Given the delicate convergence of the integrals at large $u$, the topological invariance is not obvious. We will discover that for $N_{f}<4$, the integrals are locally constant as a function of the metric and have a wall-crossing behavior similar to that of Donaldson theory. In marked contrast, for $N_{f}=4$ (and $\mathcal{N}=4$ ) the integrals have no wall-crossing, but instead have a continuous dependence on the period point $\omega$. Nevertheless, the $N_{f}=4$ theory does have a truly topological subsector. This is defined by correlation functions of observables satisfying an upper bound on the ghost number which we derive below. For these observables the integrals are absolutely convergent at infinity and have no metric dependence at all, even for $b_{2}^{+}=1$.

\footnotetext{
${ }^{24}$ In principle other regularizations are possible. For instance, one could use the coordinate $a$ at infinity and make a similar definition. We expect that any difference between the two answers could be interpreted in terms of a redefinition of the observables, along the lines suggested in section 3.2 .
} 
Let us consider a family of period points $\omega(t)$ and study $Z_{u}(\omega(t))$. The variation of the path integral with respect to the metric is given by the one-point function of the energy momentum tensor $T$. Since we have a topological field theory, the energy momentum tensor is BRST exact; that is, $T=\{\overline{\mathcal{Q}}, L\}$ for some local and duality invariant quantity $L$. When the path integral is reduced to an integral on the $u$-plane, the BRST exact integrand is expected to become a total derivative in $\bar{u}$. We will now exhibit this behavior directly from the expression (11.8).

The nonholomorphic and metric dependent factors in the integrand of (11.8) are all contained in the expression:

$$
\begin{aligned}
& \widetilde{\Psi} \equiv \frac{d \bar{\tau}}{d \bar{u}} \exp \left[\frac{1}{8 \pi y}\left(\frac{d u}{d a}\right)^{2} S_{+}^{2}\right] e^{2 \pi i \lambda_{0}^{2}} \sum_{\lambda \in H^{2}+\frac{1}{2} w_{2}(X)}(-1)^{\left(\lambda-\lambda_{0}\right) \cdot w_{2}(X)} \\
& \cdot\left[\frac{(\lambda, \omega)}{y^{1 / 2}}+\frac{i}{4 \pi y^{3 / 2}} \frac{d u}{d a}(S, \omega)\right] \cdot \exp \left[-i \pi \bar{\tau}\left(\lambda_{+}\right)^{2}-i \pi \tau\left(\lambda_{-}\right)^{2}-i \frac{d u}{d a}\left(S, \lambda_{-}\right)\right]
\end{aligned}
$$

The derivative of $\widetilde{\Psi}(\omega(t))$ with respect to $t$ can be rewritten as a total derivative $d / d \bar{u}$ :

$$
\frac{d}{d t} \widetilde{\Psi}(\omega(t))=\frac{d}{d \bar{u}} \Upsilon
$$

The explicit formula is:

$$
\begin{gathered}
\Upsilon=-4 i \exp \left[\frac{1}{8 \pi y}\left(\frac{d u}{d a}\right)^{2} S_{+}^{2}\right] e^{2 \pi i \lambda_{0}^{2}} \sum_{\lambda \in H^{2}+\frac{1}{2} w_{2}(X)}(-1)^{\left(\lambda-\lambda_{0}\right) \cdot w_{2}(X)} \\
\cdot\left[(\lambda, \dot{\omega}) y^{1 / 2}+\frac{y^{-1 / 2}}{4 \pi i} \frac{d u}{d a}(S, \dot{\omega})\right] \cdot \exp \left[-i \pi \bar{\tau}\left(\lambda_{+}\right)^{2}-i \pi \tau\left(\lambda_{-}\right)^{2}-i \frac{d u}{d a}\left(S, \lambda_{-}\right)\right] .
\end{gathered}
$$

An important feature of $\Upsilon$ is that it transforms well under modular transformations when combined with the contact term $\exp \left[S^{2} T(u)\right]$. Thus, one can integrate by parts. It is possible to write similar expressions directly for the integrand of (11.8); however, these expressions are in general not useful because they do not have good modular transformations (they are quite analogous to the second term in (9.2)).

Using (11.14), we can perform the integration by parts, and find that the continuous variation of the Coulomb branch integral is

$$
\frac{d}{d t} Z_{u}(\omega(t))=-i \alpha^{\chi} \beta^{\sigma} \lim _{R \rightarrow \infty} \oint_{|u|=R} d u\left(\frac{d a}{d u}\right)^{1-\frac{1}{2} \chi} \Delta^{\sigma / 8} e^{2 p u+S^{2} T(u)} \Upsilon
$$


Now, if $N_{f}<4, \tau(u) \rightarrow i \infty$ for $u \rightarrow \infty$, indeed, $q \sim 1 / u^{4-N_{f}}$. Hence the terms in the lattice theta function decay at infinity. If we do not cross a wall, then $\lambda_{+}^{2}>0$, so to cancel the phase integral on $u$ the integrand must go like $u^{-1}(u \bar{u})^{-\nu}$ for $\nu>0$, and hence the variation of the integral vanishes. Thus, except for the wall-crossing, the integral is topologically invariant for $N_{f}<4$.

The situation is quite different for $N_{f}=4$. Since $\tau$ approaches a constant $\tau_{0}$, there is no suppression from the lattice theta function. The integrand pertaining to the general correlation function of high order involves a sum of terms including a term $\sim \frac{d u}{u}$. Thus, there is continuous variation of the integral as a function of $\omega$ !

While for $N_{f}=4$ we have thus lost topological invariance for the general correlator at $b_{2}^{+}=1$, there is a special subclass of correlators which are completely invariant, that is, have no continuous variation or wall-crossing, and are thereby true topological invariants. These are the correlators for which the integral at $u=\infty$ is absolutely convergent. The variation (11.16) of a correlator involving $p^{\ell} S^{r}$ has an integrand which behaves for large $u$ like

$$
\oint d u u^{(\sigma+1) / 2} p^{\ell} u^{\ell} S^{r} u^{r / 2}\left[\Upsilon_{0}+\mathcal{O}(1 / u, 1 / \bar{u})\right]
$$

Here $\Upsilon_{0}$ is the limiting value of (11.15) at the appropriate order in $S$. It is generically nonzero. The term $u^{(\sigma+1) / 2}$ comes from the measure and we have used $\chi+\sigma=4$. If the leading power of $u$ is less than -1 then we cannot have any variation of the correlator, either continuous or discontinuous. Hence, correlators of ghost number $Q=4 \ell+2 r$ are true topological (or rather differentiable) invariants, even for manifolds of $b_{2}^{+}=1$ (and $b_{1}=0$ ), for

$$
6+2 \sigma+Q<0
$$

\subsection{Other Properties of the $u$-Plane Integrals}

Now we will discuss other general properties of the $u$-plane integrals. Many results we found for Donaldson theory generalize nicely, but there are some changes.

The vanishing in certain chambers of the $u$-plane integral, discussed in section 5 , does not have a precise analog for $N_{f}>0$ because of the restriction $w_{2}(E)=w_{2}(X)$, which prevents one from considering the appropriate bundles. This vanishing does have an analog for $\mathcal{N}=4$, which can be obtained in the same way. Since the twisted $\mathcal{N}=4$ theory is restricted to four-manifolds $X$ with $w_{2}(X)=0$, the only practical case of the vanishing is $\mathbb{P}^{1} \times \mathbb{P}^{1}$. 
The blow-up formula analyzed in section 6 generalizes as follows. The derivation of the blow-up formula is exactly the same as before, but for $N_{f}>0$, we must choose the case $w_{2}(\widetilde{E}) \cdot B=1 \bmod 2$ because of the restriction $w_{2}(E)=w_{2}(X)$. Following through the steps (6.3) to (6.5) we find that the integrand for the manifold $\widehat{X}$ is that of the integrand for $X$ times:

$$
\frac{\alpha}{\beta} 2^{9 / 4} t \exp \left[-t^{2} H(u)-\sum_{k=2}^{\infty} \frac{t^{2 k}}{2 k} \frac{G_{2 k}(\tau)}{(2 \pi h)^{2 k}}\right],
$$

where $h=d a / d u$. The Eisenstein functions $G_{2 k}(\tau)$ are related to Eisenstein series $G_{2 k}(L)$ of the lattice $L=\omega_{1} \mathbb{Z}+\omega_{2} \mathbb{Z}$ by

$$
\frac{G_{2 k}(\tau)}{(2 \pi h)^{2 k}}=2^{k} G_{2 k}(L)
$$

The $G_{2 k}(L)$ may be expressed as polynomials in the coefficients $a_{2}, a_{4}, a_{6}$ of (11.1). Therefore, there are universal polynomials $\mathcal{B}_{k}\left(u, a_{2}, a_{4}, a_{6}\right)$ defined by:

$$
t \exp \left[-t^{2} H(u)-\sum_{k=2}^{\infty} \frac{\left(2 t^{2}\right)^{k}}{2 k} G_{2 k}(L)\right]=\sum_{k=1}^{\infty} t^{k} \mathcal{B}_{k}\left(u, a_{2}, a_{4}, a_{6}\right),
$$

such that, for all $N_{f}$ the blow-up formula is given by:

$$
\begin{aligned}
&\langle\exp [I(S)+t I(B)+p \mathcal{O}]\rangle_{\hat{X}}= \\
& \frac{\alpha}{\beta} 2^{9 / 4} \sum_{k \geq 1} t^{k}\left\langle\exp [I(S)+p \mathcal{O}] \mathcal{B}_{k}\left(u, a_{2}, a_{4}, a_{6}\right)\right\rangle_{X}
\end{aligned}
$$

(where $w_{2}(E)=w_{2}(X)$ on both sides). We conclude that the coefficients in the generalized blow-up formula are polynomial in the masses with rational coefficients.

One can analyze wall-crossing just as in section 4, with results just like those of section 4 at zeroes of $\Delta$ (and some modifications, for reasons explained in section 11.3, near $u=\infty$ ). At a zero $u=u_{*}$ of $\Delta$, the wall crossing at $W_{\lambda}$ is:

$$
\begin{aligned}
Z_{+}-Z_{-}=2 \sqrt{2} e^{2 \pi i \lambda_{0}^{2}}(-1)^{\left(\lambda-\lambda_{0}\right) \cdot w_{2}(X)} \alpha^{\chi} \beta^{\sigma} \\
\cdot\left[q^{-\lambda^{2} / 2}\left(\frac{d u}{d \tau}\right)\left(\frac{d a}{d u}\right)^{1-\frac{1}{2} \chi} \Delta^{\sigma / 8} e^{2 p u+S^{2} T-i \frac{d u}{d a}(S, \lambda)}\right]_{q^{0}},
\end{aligned}
$$


where we expand in the good local $q$-coordinate:

$$
\begin{aligned}
u & =u_{*}+\kappa_{*} q+\mathcal{O}\left(q^{2}\right), \\
\frac{d a}{d u} & =\left(\frac{d a}{d u}\right)_{*}+\left(\frac{d^{2} a}{d u^{2}}\right)_{*}\left(u-u_{*}\right)+\cdots .
\end{aligned}
$$

Similarly, the wall-crossing at $\infty$ is given by the contour integral:

$$
\begin{aligned}
Z_{+}- & Z_{-}=2 \sqrt{2}\left(4-N_{f}\right) e^{2 \pi i \lambda \cdot \lambda_{0}} \alpha^{\chi} \beta^{\sigma} \\
& \quad \lim _{R \rightarrow \infty} \oint_{|u|=R} d u q^{-\lambda^{2} / 2}\left(\frac{d a}{d u}\right)^{1-\frac{1}{2} \chi} \Delta^{\sigma / 8} \exp \left[2 p u+S^{2} T-i \frac{d u}{d a}(S, \lambda)\right]
\end{aligned}
$$

The above wall-crossing formulae are consistent with RG flow. That is, if we take a quark mass to infinity $m^{2} \rightarrow \infty$ in a theory with $N_{f}$ quarks then the wall-crossing at $u \cong m^{2}$ combines with the wall-crossing for $N_{f}$ quarks at $u=\infty$ to produce the wall-crossing at $u=\infty$ for the theory with $N_{f}-1$ quarks. To prove this one expresses (11.23) as a contour integral in the $u$-plane and shows that it combines correctly with (11.25) to produce the corresponding expression at $N_{f}-1$ in the limit $m^{2} \rightarrow \infty$.

As in section 11.3, the main qualitative difference from what we have seen in the case $N_{f}=0$ comes in the analysis of wall-crossing at $u=\infty$ for the asymptotically conformally invariant theories $N_{f}=4$ and $\mathcal{N}=4$. In these cases, because the effective $\tau$ parameter does not diverge at $u=\infty$, the behavior near $u=\infty$ is not at all like what we encountered in section 4 . In these cases there is never any wall-crossing at $u=\infty$, but there is continuous variation with the period point $\omega$, except for those correlators satisfying (11.18). For these correlators the convergence is uniform at infinity and independent of the value of the period point $\omega$. Hence, there is no variation at all. Since all other $u$-plane wall-crossing (localized at zeroes of $\Delta$ ) will cancel SW contributions, the result is that for these correlators, one actually gets true invariants for four-manifolds of $b_{2}^{+}=1$, in contrast to the usual situation in Donaldson theory, in which one gets invariants only for $b_{2}^{+}>1$.

A similar discussion holds for the other theories at $N_{f}<4$. Using (11.25) one finds that in this case there is no wall-crossing, hence no variation of the correlators of ghost number $Q$ for:

$$
6+\frac{N_{f} \sigma}{2}+Q<0 .
$$

Since $Q \geq 0$ this phenomenon does not occur in Donaldson theory (i.e., for $\left.N_{f}=0\right)$. 


\subsection{SW Contributions}

Finally we turn to the generalization of the results of section 7 . The universal functions $C, P, L$ are obtained from (11.23) in exactly the same way as before and the result is:

$$
\begin{aligned}
& C=\left(a-a_{*}\right) / q \\
& L=-i \pi 2 \sqrt{2} \alpha^{4}\left(\frac{d u}{d a}\right)^{2}, \\
& P=-8 \pi^{2} \beta^{8} \Delta\left(a-a_{*}\right)^{-1} .
\end{aligned}
$$

Indeed, with the proper interpretation of $d a / d u$ this is the general form for all cases, at all zeroes $u_{*}$ of the discriminant.

As an application of these formulae we give the detailed form of the invariants for four-manifolds of simple type with $b_{2}^{+}>1$ and $b_{1}=0$ (thus generalizing equation 2.17 of [4] to all $N_{f}$ ) ${ }^{25}$ In this case the $u$-plane integral vanishes and the entire path integral is a sum over the SW basic classes $\lambda$ which obey $d_{\lambda}=\lambda^{2}-\frac{2 \chi+3 \sigma}{4}=0$. The contribution of each class $\lambda$ is a sum over the zeroes $u_{*}$ of the discriminant, with a given zero contributing

$$
\begin{aligned}
S W(\lambda) 2^{1+\frac{3 \sigma+\chi}{2}} e^{2 \pi i \lambda \cdot \lambda_{0}}(-i)^{\delta} & \left(\frac{\pi^{2} \beta^{8}}{2^{5}}\right)^{\sigma / 8}\left(\sqrt{2} \pi \alpha^{4}\right)^{\chi / 4} \kappa_{*}^{\delta}\left(\left(\frac{d a}{d u}\right)_{*}\right)^{-(\delta+\sigma)} \\
& \cdot \exp \left[2 p u_{*}+S^{2} T_{*}-i\left(\frac{d u}{d a}\right)_{*}(S, \lambda)\right], \quad(11.28)
\end{aligned}
$$

where $\delta=\frac{\chi+\sigma}{4}$ and $\kappa_{*}$ was defined in (11.24). Note that we can simplify

$$
T_{*}=-\frac{1}{24}\left(\left(\frac{d u}{d a}\right)_{*}^{2}-8 u_{*}\right) .
$$

Thus, the contribution is expressed solely in terms of the positions of the zeroes and the values of the periods there. Because of this, we can be more explicit in terms of the relation between (11.28) and the parameters in the SW curve (11.1). By standard reduction techniques (see, e.g., section three of [45]) (11.1) is equivalent to the curve:

$$
\begin{aligned}
& y^{2}=x^{3}-\frac{c_{4}}{48} x-\frac{c_{6}}{864}, \\
& c_{4}=16\left(a_{2}^{2}-3 a_{4}\right), \\
& c_{6}=-64 a_{2}^{3}+288 a_{2} a_{4}-864 a_{6} .
\end{aligned}
$$

\footnotetext{
${ }^{25}$ In [44], J. Labastida and M. Mariño generalized the reasoning of [4] to give the result for $N_{f}=1$ in the massless case for spin manifolds. One can check that the expression given below agrees with their result for this case.
} 
By comparing the values of Eisenstein functions we can extract $\left(\frac{d a}{d u}\right)_{*}$ up to sign from

$$
\left(\frac{d a}{d u}\right)_{*}^{2}=\frac{c_{4}\left(u_{*}\right)}{2 c_{6}\left(u_{*}\right)}
$$

Using

$$
\Delta=\frac{(2 \pi)^{12}}{\omega^{12}} \eta^{24}(\tau)=2^{-6} \frac{\eta^{24}(q)}{(d a / d u)^{12}}
$$

we then obtain:

$$
\kappa_{*}=\frac{c_{4}^{3}\left(u_{*}\right)}{\Delta^{\prime}\left(u_{*}\right)} .
$$

Note that (11.31) only determines the value of the period up to sign. In fact, we do not need to resolve the squareroot. We must sum over the contributions of the SW basic classs $\lambda$ and $-\lambda$ and hence we may average (11.28) over $\lambda$ and $-\lambda$. Since [4]

$$
S W(-\lambda)=(-1)^{\delta} S W(\lambda)
$$

the factor $\exp \left[-i\left(\frac{d u}{d a}\right)_{*}(S, \lambda)\right]$ averages to a cosine when $\delta+\sigma$ is even and to a sine when $\delta+\sigma$ is odd. This combines with the prefactor to produce a series in even powers of $\left(\frac{d u}{d a}\right)_{*}$.

When we sum (11.28) over the zeroes $u_{*}$ we obtain an expression totally symmetric in the roots of $\Delta$. Therefore, at any order in $p, S$ the invariants are rational expressions in $m_{i}, e_{i}\left(\tau_{0}\right)$ at $N_{f}=4$ and in $m_{i}, \Lambda$ for $N_{f}<4$.

Perhaps the simplest example of these new invariants is the result for $X$ a K3 surface. In this case, only $\lambda=0$ contributes. The sum over the roots becomes:

$$
\sum_{i=1, \ldots, N_{f}+2} \frac{c_{4}\left(u_{i}\right)^{4}}{\Delta^{\prime}\left(u_{i}\right)^{2} c_{6}\left(u_{i}\right)} \exp \left[\left(2 p+S^{2} / 3\right) u_{i}-\frac{S^{2}}{12}\left(c_{6}\left(u_{i}\right) / c_{4}\left(u_{i}\right)\right)\right] .
$$

We have used $c_{4}\left(u_{i}\right)^{3}=c_{6}\left(u_{i}\right)^{2}$, since $\Delta\left(u_{i}\right)=0$, and have omitted an overall function of the $m_{i}, \Lambda$.

\subsection{Other $u$-Plane Integrals}

Much of the discussion of this section, and the $u$-plane integral (11.8) in particular, makes sense for more general families of elliptic curves. Thus, for example, toroidally compactified tensionless string theories provide a family of $d=4, \mathcal{N}=2$ theories which can be twisted to produce topological field theories. The Coulomb branch of these theories is described by the $E_{8}$ curve 
of $[46,47]$. In this case there are 12 singularities in the $u$-plane and $\tau$ becomes a constant at infinity. This strongly suggests that as in the discussion of the $N_{f}=4, \mathcal{N}=4$ theories, only a finite set of correlators satisfying a condition analogous to (11.18) will give invariants.

Another extremely interesting generalization of the $u$-plane integrals might be provided by topological field theories associated with the $D 3$ probe $[48,49]$ in the context of $F$-theory [50, 51]. In this case one would integrate over the $u$-sphere, regarded as the base of an elliptically fibered K3 surface. Various quantities in (11.8) can be interpreted as sections of line bundles over the $u$-sphere and nonvanishing correlators can be identified from combinations of operators leading to a globally well-defined $(1,1)$ form. Nevertheless, while certain correlators in (11.8) apparently make sense, several open problems remain. It is not clear, for example, how to define the topological field theory whose Coulomb branch leads to (11.8). The discovery of this theory could be particularly interesting because the $u$-sphere theory, if it really exists, will obey all the axioms of topological field theory, with Hilbert spaces associated to three-manifolds and complete cut and paste rules. This is probably not the case for the other theories, even for the $N_{f}=4$ and $\mathcal{N}=4$ theories, because of the noncompactness of the $u$-plane.

\section{Conclusions}

In this paper, we have obtained a more comprehensive understanding of the relation between the Donaldson invariants and the physics of $\mathcal{N}=2$ supersymmetric Yang-Mills theory. In particular, we have explained the role of the $u$-plane in Donaldson theory more thoroughly than had been done before, both for $b_{2}^{+}=1$ and for hypothetical four-manifolds of $b_{2}^{+}>1$ that are not of simple type. We hope that in the process the power of the quantum field theory approach to Donaldson theory and the rationale for the role of modular functions in Donaldson theory have become clearer.

Our results can be summarized by an admittedly rather complicated formula for the Donaldson invariants of any simply connected compact fourmanifold with $b_{2}^{+}>0$. It is:

$$
\left\langle e^{p \mathcal{O}+I(S)}\right\rangle_{\xi}=Z_{u, \xi}+\sum_{\lambda \in H^{2}(X ; \mathbb{Z})+\frac{1}{2} w_{2}(X)}\left[\left\langle e^{p \mathcal{O}+I(S)}\right\rangle_{\xi, 1, \lambda}+\left\langle e^{p \mathcal{O}+I(S)}\right\rangle_{\xi,-1, \lambda}\right]
$$

where $Z_{u, \xi}$ is defined by equations (3.18), (3.20), and (3.21) and the SW contribution at $u=1$ is defined by (7.5) and (7.17), with a similar formula for $u=-1$. The result can be extended to non-simply- connected manifolds along the lines discussed in section 10 . 
The above considerations can be generalized and extended in several interesting ways.

It is of some interest to extend Donaldson invariants to invariants of a family of four-manifolds, valued in $H^{*}(\mathrm{BDiff}(\mathrm{X}))$ [5], and some work on wallcrossing formulae in this context has recently been done [52]. It should be possible to study family invariants, and their wall-crossing formulae (which will occur for $b_{2}^{+}>1$ ) by a relatively simple extension of the above arguments. To do so, one would include in the analysis a BRST partner of the metric $\left\{\overline{\mathcal{Q}}, g_{\mu \nu}\right\}=\psi_{\mu \nu}$, giving rise to differential forms on $\mathrm{BDiff}(\mathrm{X})$. Wall-crossing formulae should be obtained from the corresponding $u$-plane integral as above.

Another avenue for research is in the generalization of the above results to other $\mathcal{N}=2$ systems. We have indicated in section 11 how the results generalize to $S U(2)$ theories with hypermultiplets. It would also be interesting to investigate higher rank gauge groups, and to study more thoroughly the reductions of six-dimensional tensionless string theories, and their hypothetical $F$-theoretic generalizations. Some of these generalizations are currently under study.

It would also be of some interest to connect these results to nonperturbative string theory. The above results will probably have some use in working with wrapped D-branes.

\section{Acknowledgements}

We would like to thank R. Borcherds, I. Frenkel, R. Friedman, P. Kronheimer, T. Li, M. Mariño, J. Morgan, T. Mrowka, P. Sarnak, N. Seiberg, R. Stern, and G. Zuckerman for discussions and correspondence. GM would like to thank A. Losev, N. Nekrasov, and S. Shatashvili for many discussions on Donaldson theory over the years. GM would like to thank CERN and the Aspen Center for Physics for hospitality during the latter course of this work. 'The work of GM is supported by DOE grant DE-FG02-92ER40704. The work of EW is supported by NSF grant PHY-9513835.

\section{Appendices}

\section{A Elliptic Curves, Congruence Subgroups, and Modular Forms}

Here we collect some useful facts and notations related to various modular forms in the paper. 
The covariant Eisenstein function of weight two is $\widehat{E}_{2}$ where:

$$
\begin{aligned}
& E_{2}=1-24 q+\cdots, \\
& \widehat{E}_{2} \equiv E_{2}-\frac{3}{\pi y} .
\end{aligned}
$$

Our conventions for theta functions are:

$$
\begin{aligned}
\frac{\vartheta\left[\begin{array}{l}
\theta \\
\phi
\end{array}\right](0 \mid \tau)}{\eta} & =\frac{\sum q^{\frac{1}{2}(n+\theta)^{2}} e^{2 \pi i(n+\theta) \phi}}{\eta} \\
& =e^{2 \pi i \theta \phi} q^{\left(\frac{\theta^{2}}{2}-\frac{1}{24}\right)} \prod\left(1+e^{2 \pi i \phi} q^{n-\frac{1}{2}+\theta}\right)\left(1+e^{-2 \pi i \phi} q^{n-\frac{1}{2}-\theta}\right),
\end{aligned}
$$

with $0 \leq \theta, \phi \leq 1$.

In particular the three Jacobian theta functions have series and product representations:

$$
\begin{aligned}
\vartheta_{2} & =\vartheta\left[\begin{array}{c}
1 / 2 \\
0
\end{array}\right](0 \mid \tau)=2 q^{1 / 8} \prod\left(1-q^{n}\right)\left(1+q^{n}\right)^{2} \\
& =\sum_{n \in \mathbb{Z}} q^{\frac{1}{2}\left(n+\frac{1}{2}\right)^{2}}=2 q^{1 / 8}+\cdots, \\
\vartheta_{3} & =\vartheta\left[\begin{array}{l}
0 \\
0
\end{array}\right](0 \mid \tau)=\prod\left(1-q^{n}\right)\left(1+q^{n-\frac{1}{2}}\right)^{2} \\
& =\sum_{n \in \mathbb{Z}} q^{\frac{1}{2} n^{2}}=1+2 q^{\frac{1}{2}}+\cdots, \\
\vartheta_{4} & =\vartheta\left[\begin{array}{c}
0 \\
1 / 2
\end{array}\right](0 \mid \tau)=\prod\left(1-q^{n}\right)\left(1-q^{n-\frac{1}{2}}\right)^{2} \\
& =\sum_{n \in \mathbb{Z}} q^{\frac{1}{2} n^{2}}(-1)^{n}=1-2 q^{\frac{1}{2}}+\cdots .
\end{aligned}
$$

The Seiberg-Witten curve is:

$$
y^{2}=x^{3}-u x^{2}+\frac{\Lambda^{4}}{4} x
$$

If we set $\Lambda=1$ the singularities will be at: $u=1$ for the monopole cusp and $u=-1$ for the dyon cusp. This is the modular curve of $\Gamma^{0}(4)$.

The group $\Gamma^{0}(4)$ is conjugate in $G L(2, \mathbb{Q})$ to the subgroup $\Gamma(2)$ of $S L(2, \mathbb{Z})$ which consists of matrices congruent to 1 modulo 2 . The $u$-plane could equally well be identified (as in [2]) as the modular curve of $\Gamma(2)$, which parametrizes a family of elliptic curves $C_{u}^{\prime}$, defined by $y^{2}=\left(x^{2}-1\right)(x-u)$, with a distinguished level two structure (the points with $y=0$ and $x=$ $1,-1, u)$. The two families of elliptic curves differ by a two-isogeny. We use 
here (as in [3]) the $\Gamma^{0}(4)$ description, to make some formulas slightly more natural and to facilitate comparison to the mathematical literature. The translation between the two descriptions is given by:

$$
\begin{aligned}
u & =\widetilde{u}, \\
\tau & =2 \widetilde{\tau}, \\
a & =\widetilde{a} / 2, \\
a_{D} & =\widetilde{a}_{D} .
\end{aligned}
$$

where quantities in the $\Gamma(2)$ description are denoted with a tilde.

In terms of theta functions we have:

$$
\begin{aligned}
u & =\frac{1}{2} \frac{\vartheta_{2}^{4}+\vartheta_{3}^{4}}{\left(\vartheta_{2} \vartheta_{3}\right)^{2}}, \\
u^{2}-1 & =\frac{1}{4} \frac{\vartheta_{4}^{8}}{\left(\vartheta_{2} \vartheta_{3}\right)^{4}}=\frac{\vartheta_{4}^{8}}{64 h^{4}(\tau)}, \\
\frac{i}{\pi} \frac{d u}{d \tau} & =\frac{1}{4} \frac{\vartheta_{4}^{8}}{\left(\vartheta_{2} \vartheta_{3}\right)^{2}}, \\
\left(\frac{\left(\frac{2 i}{\pi} \frac{d u}{d \tau}\right)^{2}}{u^{2}-1}\right)^{1 / 8} & =\vartheta_{4}, \\
h(\tau) & =\frac{d a}{d u}=\frac{1}{2} \vartheta_{2} \vartheta_{3} .
\end{aligned}
$$

The following $q$-expansions are sometimes useful:

$$
\begin{aligned}
u=u_{(\infty, 0)}= & \frac{1}{8 q^{1 / 4}}\left(1+20 q^{1 / 2}-62 q+216 q^{3 / 2}+\cdots\right) \\
= & \frac{1}{8 q^{\frac{1}{4}}}+\frac{5 q^{\frac{1}{4}}}{2}-\frac{31 q^{\frac{3}{4}}}{4}+27 q^{\frac{5}{4}}-\frac{641 q^{\frac{7}{4}}}{8}+\frac{409 q^{\frac{9}{4}}}{2}+\cdots \\
u_{(\infty, 1)}= & -\frac{i}{8 q^{1 / 4}}+\frac{5 i}{2} q^{\frac{1}{4}}+\frac{31 i}{4} q^{\frac{3}{4}} \\
& +27 i q^{\frac{5}{4}}+\frac{641 i}{8} q^{\frac{7}{4}}+\frac{409 i}{2} q^{\frac{9}{4}}+\cdots, \\
u_{M}\left(q_{D}\right)= & 1+32 q_{D}+256 q_{D}^{2} \\
& +1408 q_{D}^{3}+6144 q_{D}^{4}+22976 q_{D}^{5}+76800 q_{D}^{6}+\cdots
\end{aligned}
$$




$$
\begin{aligned}
T(u)= & -\frac{1}{24}\left[\frac{E_{2}}{h(\tau)^{2}}-8 u\right] \\
= & q^{1 / 4}-2 q^{3 / 4}+6 q^{5 / 4}-16 q^{7 / 4}+37 q^{9 / 4}-78 q^{11 / 4} \\
& +158 q^{13 / 4}-312 q^{15 / 4}+594 q^{17 / 4}+\cdots
\end{aligned}
$$

Finally, near the monopole cusp we have:

$$
a_{D}\left(q_{D}\right)=16 i q_{D}\left(1+6 q_{D}+24 q_{D}^{2}+76 q_{D}^{3}+\cdots\right) .
$$

\section{B Siegel-Narain Theta functions}

Let $\Lambda$ be a lattice of signature $\left(b_{+}, b_{-}\right)$. Let $P$ be a decomposition of $\Lambda \otimes \mathbb{R}$ as a sum of orthogonal subspaces of definite signature:

$$
P: \Lambda \otimes \mathbb{R} \cong \mathbb{R}^{b_{+}, 0} \perp \mathbb{R}^{0, b_{-}}
$$

Let $P_{ \pm}(\lambda)=\lambda_{ \pm}$denote the projections onto the two factors. We also write $\lambda=\lambda_{+}+\lambda_{-}$. With our conventions $P_{-}(\lambda)^{2} \leq 0$.

Let $\Lambda+\gamma$ denote a translate of the lattice $\Lambda$. We define the Siegel-Narain theta function

$$
\begin{aligned}
& \Theta_{\Lambda+\gamma}(\tau, \alpha, \beta ; P, \xi) \equiv \exp \left[\frac{\pi}{2 y}\left(\xi_{+}^{2}-\xi_{-}^{2}\right)\right] \\
& \cdot \sum_{\lambda \in \Lambda+\gamma} \exp \left\{i \pi \tau(\lambda+\beta)_{+}^{2}+i \pi \bar{\tau}(\lambda+\beta)_{-}^{2}+2 \pi i(\lambda+\beta, \xi)-2 \pi i\left(\lambda+\frac{1}{2} \beta, \alpha\right)\right\} \\
& =e^{i \pi(\beta, \alpha)} \exp \left[\frac{\pi}{2 y}\left(\xi_{+}^{2}-\xi_{-}^{2}\right)\right] \\
& \cdot \sum_{\lambda \in \Lambda+\gamma} \exp \left\{i \pi \tau(\lambda+\beta)_{+}^{2}+i \pi \bar{\tau}(\lambda+\beta)_{-}^{2}+2 \pi i(\lambda+\beta, \xi)-2 \pi i(\lambda+\beta, \alpha)\right\} .
\end{aligned}
$$


We modify slightly the definitions in [11] for the present case: There is no essential simplification in passing to an even sublattice. We also treat insertions somewhat differently.

The main transformation law is:

$$
\Theta_{\Lambda}\left(-1 . / \tau, \alpha, \beta ; P, \frac{\xi_{+}}{\tau}+\frac{\xi_{-}}{\bar{\tau}}\right)=\sqrt{\frac{|\Lambda|}{\left|\Lambda^{\prime}\right|}}(-i \tau)^{b_{+} / 2}(i \bar{\tau})^{b_{-} / 2} \Theta_{\Lambda^{\prime}}(\tau, \beta,-\alpha ; P, \xi)
$$

where $\Lambda^{\prime}$ is the dual lattice. If there is a characteristic vector, call it $w_{2}$, such that

$$
(\lambda, \lambda)=\left(\lambda, w_{2}\right) \bmod 2,
$$

for all $\lambda$ then we have in addition:

$$
\Theta_{\Lambda}(\tau+1, \alpha, \beta ; P, \xi)=e^{-i \pi\left(\beta, w_{2}\right) / 2} \Theta_{\Lambda}\left(\tau, \alpha-\beta-\frac{1}{2} w_{2}, \beta ; P, \xi\right) .
$$

\section{Some Details in the Derivation of (8.9)}

The strategy to do the integral was explained below (8.6). We must evaluate two terms separately, called the degenerate and nondegenerate orbits.

\section{C.1 The Nondegenerate Orbit}

After transforming the integration to the strip $\mathcal{S}$ and setting $d=0$, we simplify the $y$-dependent terms in the exponentials to get:

$$
\begin{gathered}
\frac{1}{\sqrt{2 z_{+}^{2}}} \int_{\mathcal{S}} \frac{d x d y}{y^{2}} \sum_{I} f_{I} \sum_{j=-\infty}^{\infty} ' \exp \left\{-\frac{1}{y} \frac{\pi}{2 z_{+}^{2} h_{I}^{2}}\right. \\
\cdot\left(j h_{I}-\frac{1}{2 \pi}\left(\omega, z_{+}\right)\left[\left(S_{+}, \omega\right)-\frac{\left(S_{-}, z_{-}\right)}{\left(\omega, z_{+}\right)}\right]\right) \\
\left.\cdot\left(j h_{I}+\frac{1}{2 \pi}\left(\omega, z_{+}\right)\left[\left(S_{+}, \omega\right)+\frac{\left(S_{-}, z_{-}\right)}{\left(\omega, z_{+}\right)}\right]\right)\right\} \\
\cdot \sum_{\lambda \in K} \exp \left\{-i \pi \tau\left(\lambda+\beta^{I}\right)^{2}-i\left(\lambda+\beta^{I}, \widetilde{P}(S)_{-} / h_{I}\right)+2 \pi i\left(\lambda+\beta^{I}, \alpha^{I}+j \mu\right)\right\} \\
\left.\cdot\left[\left(S_{+}, \omega\right)-\frac{2 \pi}{\left(\omega, z_{+}\right)}\left[h_{I} j+\frac{\left(S_{-}, z_{-}\right)}{2 \pi}\right]\right], \quad \text { (C. } 1\right)
\end{gathered}
$$

where we have renamed $c \rightarrow j$, and the prime on the sum means we omit the term $j=0$. 
The integrand is now written as a function of $q$ and of $y$. If we first integrate over the $x$ variable then we isolate the power $q^{0}$. We can then integrate over the $y$ variable. There is a nice cancellation and the integral becomes

$$
\begin{aligned}
& -2 \sqrt{2}\left[\sum_{I} \sum_{\lambda \in K} f_{I} h_{I} \sum_{j=-\infty}^{\infty} \frac{\exp \left\{2 \pi i\left(\lambda+\beta^{I}, \mu\right) j\right\}}{j+(S, z) /\left(2 \pi h_{I}\right)}\right. \\
& \left.\cdot \exp \left\{-i \pi \tau\left(\lambda+\beta^{I}\right)^{2}-i\left(\lambda+\beta^{I}, \widetilde{P}(S)_{-}\right) / h_{I}+2 \pi i\left(\lambda+\beta^{I}, \alpha^{I}\right)\right\}\right]_{q^{0}} .
\end{aligned}
$$

The sum over $j$ can be done using the identity:

$$
\sum_{j=-\infty}^{\infty}{ }^{\prime} \frac{e^{i \theta j}}{j+A}=-\frac{1}{A}+2 \pi i \frac{e^{-i A \theta}}{1-e^{-2 \pi i A}},
$$

which is valid for

$$
0<\theta<2 \pi, \quad A \notin \mathbb{Z} .
$$

In our case we can apply this formula with the angle:

$$
\theta=2 \pi((\lambda+\beta, \mu)-[(\lambda+\beta, \mu)]), \quad 0 \leq \theta<2 \pi,
$$

where [.] is the greatest integer. Moreover, define

$$
A_{I} \equiv \frac{(S, z)}{2 \pi h_{I}} .
$$

Now we can begin to see some topological invariance. We can combine exponentials using the identity:

$$
\begin{aligned}
\widetilde{P}(S)_{-}+(S, z) \mu & =S^{K}+\frac{\left(S, z_{+}-z_{-}\right)}{2 z_{+}^{2}} z \\
S^{K} & \equiv S-(S, z) z^{\prime}
\end{aligned}
$$

On the right hand side of (C.7) $S^{K}$ is topological, and projects to $K$. The second term is metric dependent and changes continuously within chambers, but has zero inner product with all vectors in $K$. Define an angle:

$$
\psi_{I} \equiv \frac{1}{h_{I}}\left[\left(\lambda+\beta_{I}, S^{K}\right)-\left[\left(\lambda+\beta_{I}, \mu\right)\right](S, z)\right] .
$$


Using this (C.2) becomes:

$$
\begin{aligned}
& -2 \sqrt{2}\left[\sum_{I} \sum_{\lambda \in K} f_{I} h_{I} \exp \left[-i \pi \tau\left(\lambda+\beta^{I}\right)^{2}+2 \pi i\left(\lambda+\beta^{I}, \alpha^{I}\right)\right]\right. \\
& \left.\cdot\left\{-\frac{2 \pi h_{I}}{(S, z)} \exp \left(-i\left(\lambda+\beta^{I}, \widetilde{P}(S)_{-}\right) / h_{I}\right)+\frac{2 \pi i}{1-e^{-2 \pi i A_{I}}} e^{-i \psi_{I}}\right\}\right]_{q^{0}} .
\end{aligned}
$$

The expression in curly brackets in (C.9) is a sum of two terms. The first is not topological and varies continuously with metric within chambers, while the second term is a nice topological expression, within each chamber. Equation (C.9) should be regarded as a formal series in $S$. The pole terms cancel between topological and nontopological pieces.

\section{C.2 The Degenerate Orbit}

Returning to (8.5) we consider the term with $c=d=0$ :

$$
\frac{1}{\sqrt{2 z_{+}^{2}}} \int_{\mathcal{F}} \frac{d x d y}{y^{2}} \sum_{I} \widehat{f}_{I} \bar{\Theta}_{\left(\Gamma+\beta^{I}\right) \cap z^{\perp} / z}\left(\tau, \alpha^{I}, 0 ; \widetilde{P}\left(\xi^{I}\right)\right)\left[\left(S_{+}, \omega\right)-\frac{\left(S_{-}, z_{-}\right)}{\left(\omega, z_{+}\right)}\right] \text {. }
$$

Using the identities

$$
S^{2}-\left(\widetilde{P}(S)_{-}\right)^{2}=S_{+}^{2}-\frac{\left(S_{-}, z_{-}\right)^{2}}{z_{+}^{2}}=(S, z)\left(S, z_{+}-z_{-}\right) \frac{1}{z_{+}^{2}}
$$

and

$$
\left[\left(S_{+}, \omega\right)-\frac{\left(S_{-}, z_{-}\right)}{\left(\omega, z_{+}\right)}\right]=\frac{1}{\sqrt{z_{+}^{2}}}\left(S, z_{+}-z_{-}\right)
$$

and isolating the $1 / y$ dependence in the exponential we find that the integrand can be written as a total derivative $\frac{d}{d \bar{\tau}}$ of a modular invariant expression. In the standard way only the constant term at $\tau \rightarrow i \infty$ contributes. Note that we must work with formal series expressions in $S$.

Doing the integral by parts we find a metric-dependent expression:

$$
\begin{aligned}
& -4 \pi \sqrt{2} \frac{1}{(S, z)}\left[\sum_{I} f_{I} h_{I}^{2} \sum_{\lambda \in K}\right. \\
& \left.\cdot \exp \left\{-i \pi \tau\left(\lambda+\beta^{I}\right)^{2}+2 \pi i\left(\lambda+\beta^{I}, \alpha^{I}\right)-i\left(\lambda+\beta^{I}, \widetilde{P}(S)_{-}\right) / h_{I}\right\}\right]_{q^{0}} .
\end{aligned}
$$

This metric-dependent expression exactly cancels the metric-dependent term in the nondegenerate orbit! 


\section{References}

[1] E. Witten, Comm. Math. Phys. 117 (1988) 353.

[2] N. Seiberg and E. Witten, Nucl. Phys. B426 (1994) 19.

[3] N. Seiberg and E. Witten, Nucl. Phys. B431 (1994) 484.

[4] E. Witten, Math. Res. Lett. 1 (1994) 769.

[5] S. K. Donaldson, Bull. Amer. Math. Soc. 33 (1996) 45.

[6] S. Donaldson, Topology 29 (1990) 257.

[7] R. Friedman and J. W. Morgan, "Smooth Four-Manifolds and Complex Surfaces", Springer-Verlag 1991.

[8] S. K. Donaldson and P. B. Kronheimer, "The Geometry of FourManifolds", Clarendon Press 1990.

[9] P. Kronheimer and T. Mrowka, Bull. Am. Math. Soc. 30 (1994) 215; J. Diff. Geom. 33 (1995) 573.

[10] L. Göttsche and D. Zagier, "Jacobi Forms and the Structure of Donaldson Invariants for 4-Manifolds With $b_{+}=1$ ", alg-geom/9612020

[11] R. Borcherds, "Automorphic Forms With Singularities on Grassmannians", alg-geom/9609022

[12] M. Kontsevich, Sem. Bourbaki (1996) no. 821.

[13] J. A. Harvey and G. Moore, Nucl. Phys. B463 (1996) 315.

[14] S. Donaldson, J. Diff. Geom. 26 (1987) 141.

[15] L. Göttsche, J. Amer. Math. Soc. 9 (1996) 827.

[16] Z. Qin, Topology 32 (1993) 551.

[17] H. J. Hoppe and H. Spindler, Math. Ann. 249 (1980) 127.

[18] J. Morgan and Z. Szabó, "Embedded Tori In Four-Manifolds", preprint (1996).

[19] R. Fintushel and R. J. Stern, Annals of Math. 143 (1996) 529.

[20] J. Polchinski, Comm. Math. Phys. 104 (1986) 37.

[21] B. McClain and B. D. B. Roth, Comm. Math. Phys. 111 (1987) 539. 
[22] L. Dixon, V. S. Kaplunovsky and J. Louis, Nucl. Phys. B329 (1990) 27.

[23] D. Zagier, C. R. Acad. Sc. Paris, 281A (1975) 883.

[24] F. Hirzebruch and D. Zagier, Inv. Math. 36 (1976) 57.

[25] G. Ellingsrud and L. Göttsche, "Wall-Crossing Formulas, Bott Residue Formula and the Donaldson Invariants of Rational Surfaces", alggeom/9506019.

[26] A. Losev, N. Nekrasov and S. Shatashvili, to appear.

[27] D. Anselmi and P. Fré, Nucl. Phys. B416 (1994) 255; Phys. Lett. B347 (1995) 247.

[28] J. M. F. Labastida and M. Mariño, Phys. Lett. B351 (1995) 146; Nucl. Phys. B448 (1995) 373; Comm. Math. Phys. 185 (1997) 37.

[29] S. Hyun, J. Park and J. S. Park, Nucl. Phys. B453 (1995) 199.

[30] J. M. F. Labastida and P. M. Llatas, Nucl. Phys. B379 (1992) 220; M. Alvarez and J. M. F. Labastida, Nucl. Phys. B437 (1995) 356; J. M. F. Labastida and C. Lozano, "Lectures on Topological Quantum Field Theory", hep-th/9709192

[31] E. Witten, J. Math. Phys. 35 (10) 5101.

[32] S. J. Gates, Jr., Nucl. Phys. B238 (1984) 349.

[33] E. Witten, Selecta Mathematica 1 (1995) 383.

[34] L. Rozansky and E. Witten, "Hyper-Kähler Geometry and Invariants of Three-Manifolds", hep-th/9612216.

[35] S. Donaldson, J. Diff. Geom. 26 (1987) 397.

[36] E. Verlinde, Nucl. Phys. B455 (1995) 211.

[37] I. Antoniadis, S. Ferrara and T. R. Taylor, Nucl. Phys. B460 (1996) 489.

[38] M. Eichler and D. Zagier, "The Theory of Jacobi Forms", Birkhäuser 1985.

[39] H. Cohen, Math. Ann. 217 (1975) 271.

[40] T. J. Li and A. Liu, Math. Res. Lett. 2 (1995) 797. 
[41] C. Okonek and A. Teleman, Int. J. Math. 7 (1996) 811.

[42] C. Vafa and E. Witten, Nucl. Phys. B431 (1994) 3.

[43] M. Matone, Phys. Rev. D53 (1996) 7354; G. Bonelli and M. Matone, Phys. Rev. Lett. 76 (1996) 4107; M. Matone, Phys. Rev. Lett. 78 (1997) 1412.

[44] J. M. F. Labastida and M. Mariño, Nucl. Phys. B456 (1995) 633.

[45] See e.g. D. Husemöller, "Elliptic Curves", Springer 1987.

[46] O. Ganor, Nucl. Phys. B488 (1997) 223.

[47] O. Ganor, D. R. Morrison and N. Seiberg, Nucl. Phys. B487 (1997) 93.

[48] A. Sen, Nucl. Phys. B475 (1996) 562.

[49] T. Banks, M. R. Douglas and N. Seiberg, Phys. Lett. B387 (1996) 278.

[50] C. Vafa, Nucl. Phys. B469 (1996) 403.

[51] D. Morrison and C. Vafa, Nucl. Phys. B473 (1996) 74; Nucl. Phys. B476 (1996) 437.

[52] T. Li and J. Liu, to appear. 